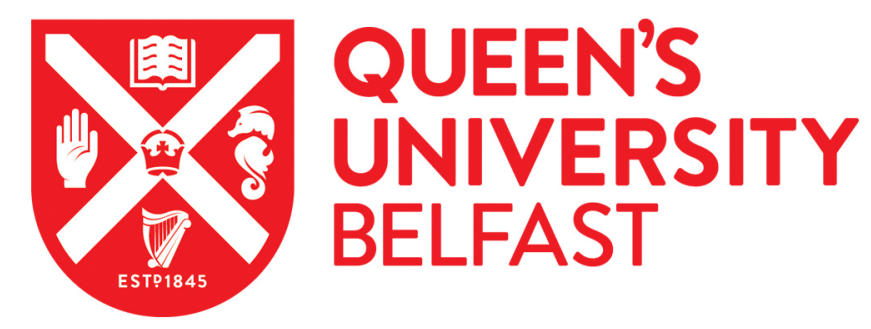

\title{
Identification of 19 new risk loci and potential regulatory mechanisms influencing susceptibility to testicular germ cell tumor
}

Litchfield, K., Levy, M., Orlando, G., Loveday, C., Law, P. J., Migliorini, G., Holroyd, A., Broderick, P., Karlsson, R., Haugen, T. B., Kristiansen, W., Nsengimana, J., Fenwick, K., Assiotis, I., Kote-Jarai, ZS., Dunning, A. M., Muir, K. R., Peto, J., Eeles, R., ... UK Testicular Cancer Collaboration (2017). Identification of 19 new risk loci and potential regulatory mechanisms influencing susceptibility to testicular germ cell tumor. Nature Genetics, 49(7), 1133-1140. https://doi.org/10.1038/ng.3896

Published in:

Nature Genetics

Document Version:

Peer reviewed version

Queen's University Belfast - Research Portal:

Link to publication record in Queen's University Belfast Research Portal

\section{Publisher rights}

(c) 2017 Nature America Inc.

This work is made available online in accordance with the publisher's policies. Please refer to any applicable terms of use of the publisher.

\section{General rights}

Copyright for the publications made accessible via the Queen's University Belfast Research Portal is retained by the author(s) and / or other copyright owners and it is a condition of accessing these publications that users recognise and abide by the legal requirements associated with these rights.

Take down policy

The Research Portal is Queen's institutional repository that provides access to Queen's research output. Every effort has been made to ensure that content in the Research Portal does not infringe any person's rights, or applicable UK laws. If you discover content in the Research Portal that you believe breaches copyright or violates any law, please contact openaccess@qub.ac.uk. 


\section{Identification of 19 new risk loci and potential regulatory mechanisms influencing susceptibility to}

testicular germ cell tumor

Kevin Litchfield ${ }^{1}$, Max Levy ${ }^{1}$, Giulia Orlando ${ }^{1}$, Chey Loveday ${ }^{1}$, Philip Law ${ }^{1}$, Gabriele Migliorini ${ }^{1}$, Amy Holroyd ${ }^{1}$, Peter Broderick ${ }^{1}$, Robert Karlsson ${ }^{2}$, Trine B Haugen ${ }^{3}$, Wenche Kristiansen ${ }^{3}$, Jérémie Nsengimana ${ }^{4}$, Kerry Fenwick ${ }^{5}$, loannis Assiotis ${ }^{5}$, ZSofia Kote-Jarai $^{1}$, Alison M. Dunning ${ }^{6}$, Kenneth Muir $^{8,9}$, Julian Peto ${ }^{10}$, Rosalind Eeles ${ }^{1,11}$, Douglas F Easton ${ }^{6,7}$, Darshna Dudakia ${ }^{1}$, Nick Orr ${ }^{12}$, Nora Pashayan $^{13}$, UK Testicular Cancer Collaboration*, The PRACTICAL consortium*, D. Timothy Bishop ${ }^{4}$, Alison Reid ${ }^{14}$, Robert A Huddart ${ }^{14}$, Janet Shipley ${ }^{15}$, Tom Grotmol ${ }^{16}$, Fredrik Wiklund ${ }^{2}$, Richard S Houlston ${ }^{1}$, Clare Turnbull ${ }^{1,17}$

1. Division of Genetics \& Epidemiology, The Institute of Cancer Research, London, SM2 5NG, UK

2. Department of Medical Epidemiology and Biostatistics, Karolinska Institutet, Stockholm, 171 77, Sweden

3. Faculty of Health Sciences, Oslo and Akershus University College of Applied Sciences, Oslo, Norway

4. Section of Epidemiology \& Biostatistics, Leeds Institute of Cancer and Pathology, Leeds, LS9 7TF, UK

5. Tumour Profiling Unit, The Institute of Cancer Research, London, SM2 5NG, UK

6. Centre for Cancer Genetic Epidemiology, Department of Oncology, University of Cambridge, Cambridge, CB1 8RN, UK

7. Centre for Cancer Genetic Epidemiology, Department of Public Health and Primary Care, University of Cambridge, Cambridge, CB1 8RN, UK

8. Division of Health Sciences, Warwick Medical School, Warwick University, CV4 7AL, UK

9. Institute of Population Health, University of Manchester, M1 3BB, UK

10. Department of Non-Communicable Disease Epidemiology, London School of Hygiene and Tropical Medicine, London, United Kingdom. 
11. Royal Marsden NHS Foundation Trust, London, SM2 5NG, UK

12. The Breast Cancer Now Toby Robins Research Centre, The Institute of Cancer Research, 237 Fulham Road, London SW3 6JB, UK

13. Department of Applied Health Research, University College London, London, WC1E 6BT, UK

14. Academic Radiotherapy Unit, Institute of Cancer Research, Sutton, Surrey, SM2 5NG, UK

15. Division of Molecular Pathology, The Institute of Cancer Research, London, SM2 5NG, UK

16. Department of Research, Cancer Registry of Norway, Oslo, 0369, Norway

17. William Harvey Research Institute, Queen Mary University, London, EC1M 6BQ , UK

* See supplementary notes 1 and 2

Correspondence to: Clare Turnbull, Division of Genetics and Epidemiology, The Institute of Cancer Research, London, SM2 5NG, UK; Tel: ++44 (0) 208722 4485; E-mail: clare.turnbull@icr.ac.uk

Key words: Testicular Cancer, Germ Cell Tumour, TGCT, GWAS, Oncoarray. 
Genome-wide association studies (GWAS) have transformed our understanding of testicular germ cell tumour (TGCT) susceptibility but much of the heritability remains unexplained. Here we report a new GWAS, a meta-analysis with previous GWAS and a replication series, totalling 7,319 TGCT cases and 23,082 controls. We identify 19 new TGCT risk loci, approximately doubling the number of known TGCT risk loci to 44. By performing in-situ Hi-C in TGCT cells, we provide evidence for a network of physical interactions between all 44 TGCT risk SNPs and candidate causal genes. Our findings reveal widespread disruption of developmental transcriptional regulators as a basis of TGCT susceptibility, consistent with failed primordial germ cell differentiation as an initiating step in oncogenesis ${ }^{1}$. Defective microtubule assembly and dysregulation of KIT-MAPK signalling also feature as recurrently disrupted pathways. Our findings support a polygenic model of risk and provide insight into the biological basis of TGCT. 
Testicular germ cell tumour (TGCT) is the most common cancer in men aged $18-45$, with over 52,000 new cases diagnosed annually worldwide ${ }^{2}$. The development of TGCT is strongly influenced by inherited genetic factors, which contributes to nearly half of all disease risk ${ }^{3}$ and is reflected in the 4to-8 fold increased risk shown in siblings of cases $^{4-7}$. Our understanding of TGCT susceptibility has been transformed by recent genome-wide association studies (GWAS), which have so far identified 25 independent risk loci for $\mathrm{TGCT}^{8-18}$. Although projections indicate that additional risk variants for TGCT can be discovered by $\mathrm{GWAS}^{19}$, studies to date have been based on comparatively small sample sizes which have had limited power to detect common risk variants ${ }^{20}$.

To gain a more comprehensive insight into TGCT aetiology we performed a new GWAS with substantially increased power, followed by a meta-analysis with existing GWAS and replication genotyping (totalling 7,319 cases/23,082 controls). Here we report both the discovery of 19 new TGCT susceptibility loci and refined risk estimates for the previously reported loci. In addition, we have investigated the gene regulatory mechanisms underlying the genetic associations observed at all 44 TGCT GWAS risk loci by performing in-situ chromosome conformation capture in TGCT cells ( $\mathrm{Hi}-\mathrm{C})$ to characterize chromatin interactions between predisposition SNPs and target genes, integrating these data with a range of publicly available TGCT functional genomics data.

We conducted a new GWAS using the Oncoarray platform (3,206 UK TGCT cases/7,422 UK controls), followed by a meta-analysis combining the two largest published TGCT GWAS datasets ${ }^{11,16}$ (986 UK cases/4,946 UK controls, 1,327 Scandinavian cases/6,687 Scandinavian controls) (Fig. 1). To increase genomic resolution, we imputed $>10$ million SNPs using the 1000 Genomes Project as a reference panel. Quantile-Quantile (Q-Q) plots for SNPs with minor allele frequency (MAF) >5\% post imputation did not show evidence of substantive over-dispersion $\left(\lambda_{1000}=1.03\right.$, Supplementary Fig. 1$)$. We derived joint odds ratios (ORs) and 95\% confidence intervals (Cls) under a fixed-effects model for each SNP with MAF $>0.01$. Finally we sought validation of 37 SNPs associated at $P<5.0 \times 10^{-6}$, which 
did not map to known TGCT risk loci and displayed a consistent OR across all GWAS datasets, by genotyping an additional 1,801 TGCT cases and 4,027 controls from the UK. After meta-analysis of the three GWAS and replication series, we identified genome-wide significant associations (i.e. $P<5$ $\times 10^{-8}$ ) at 19 new loci (Table 1 ). We found no evidence for significant interactions between risk loci. To the extent that they have been deciphered, many GWAS risk loci map to non-coding regions of the genome and influence gene regulation. Across the 44 independent TGCT risk loci (19 new and 25 previously reported), we confirmed a significant enrichment of enhancer/promoter associated histone marks, including H3K4me1, H3K4me3 and H3K9ac, using available ChIP-Seq data from the TGCT cell line NTERA2 $\left(P<5.0 \times 10^{-3}\right)$ (Supplementary Table 1). Moreover this enrichment showed tissue specificity when compared to 41 other cell lines from the ENCODE ${ }^{21}$ project (Supplementary

Fig. 2). These observations support the assertion that the TGCT predisposition loci influence risk through effects on cis-regulatory networks, and are involved in transcriptional initiation and enhancement. Since genomic spatial proximity and chromatin looping interactions are fundamental for regulation of gene expression we performed in situ capture Hi-C of promoters in NTERA2 cells to link risk loci to candidate target genes. We also sought to gain insight into the possible biological mechanisms for the associations by performing tissue-specific expression quantitative trait loci (eQTL) analysis for all risk SNP and target gene pairs (Supplementary Fig. 3, Supplementary Table 2). We analysed RNA-seq data from both normal testis (GTEx project ${ }^{22}$ ) and TGCT (TCGA), acknowledging that the latter may be affected by the issue of tumour purity, in addition to dysregulated gene expression that typifies cancer. Accepting this limitation and that further validation may be required, eQTL analysis was conducted in both datasets based on the established network of enhancer/ promoter variants, to maximise our ability to find statistically significant associations after correcting for multiple testing. We additionally annotated risk loci with variants predicted to disrupt binding motifs of germ cell specific transcription factors (TF) (see methods). Finally, direct promoter variants and non-synonymous coding mutations for genes within the 44 risk loci were denoted (Table 2, Fig. 2). 
Although preliminary and requiring functional validation, three candidate disease mechanisms emerge from analysis across the 44 loci. Firstly, 10 of the risk loci contain candidate genes linked to developmental transcriptional regulation, as evidenced by Hi-C looping interactions (at 8p23.1, 20q13.2), eQTL effects (at 4q22.3, 8p23.1), promoter variants (at 8q13.3, 9p24.3, 12q15, 17q12, 19p12) and coding variants (at 2p13.3, 16q24.2) (Table 2). Notably the new TGCT risk locus at 8p23.1 features a looping chromatin interaction from risk SNP rs17153755 to the promoter of GATA4, which is supported by an overlapping predicted strong enhancer region and a nominal eQTL effect (TCGA data, $P=3.1 \times 10^{-2}$ ) (Fig. 3a). The rs17153755 risk allele was associated with down-regulation of GATA4 expression, consistent with the hypothesised role of GATA4 as a tumor suppressor gene $\mathrm{e}^{23,24}$. In addition the risk locus at 16q24.2 only contains a single gene ZFPM1 (alias FOG, Friend of GATA1), which encodes an essential regulator of GATA1 ${ }^{25}$, in which we noted a predicted damaging ${ }^{26}$ missense polymorphism (rs3751673, NP_722520.2:p.Arg22Gly). The GATA family of transcription factors are expressed throughout postnatal testicular development ${ }^{27}$, and play a key role in ensuring correct tissue specification and differentiation ${ }^{28}$. We also observed promoter variants at $8 \mathrm{q} 13.3$ and 9p24.3, providing support respectively for the role of PRDM14 and DMRT1 in TGCT oncogenesis, both of which encode important transcriptional regulators of germ cell specification and sex determination ${ }^{29-32}$. Of final note the new locus at $20 q 13.2$ was characterized by a predicted disrupted POU5F1 binding motif, together with a looping Hi-C contact from risk SNP rs12481572 to the promoter of SALL4, a gene associated with the maintenance of pluripotency in embryonic stem cells $s^{33}$.

Secondly, candidate genes with roles related to microtubule/chromosomal assembly were implicated at five TGCT risk loci, supported by Hi-C looping interactions (at 1q22, 15q25.2), eQTL effects (at 15q25.2, 17q22), promoter variants (at 1q22, 4q24) and coding variants (at 21q22.3). Notably at locus $17 q 22$ we observed a promoter variant (rs302875) which displays a strong eQTL 
effect (GTEx data, $P=4.9 \times 10^{-7}$ ) on TEX14 (Testis-Expressed 14), which encodes an important regulator of kinetochore-microtubule assembly in testicular germ cells ${ }^{14,34,35}$. At new risk locus $15 q 25.2$ we identified a nominal eQTL association ( $r$ s2304416, TCGA data, $P=3.2 \times 10^{-2}$ ) and accompanying chromatin looping interaction with mitotic spindle assembly related gene $W D R 73^{36}$ (Fig. 3b). WDR73 encodes a protein with a crucial role in the regulation of microtubule organization during interphase ${ }^{37}$ and biallelic mutations cause Galloway-Mowat Syndrome, a human syndrome of nephrosis and neuronal dysmigration. Finally the functional analysis also highlighted microtubule assembly related genes PMF1, CENPE and $P C N T^{38-41}$ as candidates at $1 \mathrm{q} 22,4 \mathrm{q} 24$ and $21 \mathrm{q} 22.3$ respectively.

Thirdly, the central role of KIT-MAPK signalling in TGCT oncogenesis was further supported at four loci, by Hi-C looping interactions (at 11q14.1, 15q22.31), eQTL effects (at 6p21.31) and promoter variants (at 6p21.31, 11q14.1, 15q22.31). Recent tumour sequencing studies have established that KIT is the major somatic driver gene for $\mathrm{TGCT}^{42}$ and a relationship between the previously identified risk SNP rs995030 (12q21) and KITLG expression has been demonstrated through allele-specific p53 binding by Zeron-Medina et $\mathrm{al}^{43}$. Here we report a new locus at $15 q 22.31$, containing a variant within the promoter of MAP2K1 (Fig. 3c), which raises the prospect of further elucidating mechanisms of KIT-MAPK signalling in driving TGCTs. MAP2K1 (alias MEK1) is downstream of c-Kit and MEK1 inhibition slows primordial germ cell growth in the presence of KIT ligand ${ }^{44}$. If MAP2K1 is confirmed as a causal gene at $15 q 22.31$, the study of somatic KIT mutational status in patients carrying the risk allele at $15 q 22.31$ should be highly informative. In addition, within the 11q14.1 risk locus, we identify a candidate promoter variant for GAB2, which encodes a docking protein for signal transduction to MAPK and PI3K pathways which interacts directly with $\mathrm{KIT}^{45}$. Finally in our analysis we identify both a candidate promoter variant and a nominal eQTL effect for BAK1 (6p21.31)(TCGA data, $P=1.9 \times 10^{-2}$ ), which encodes a protein regulating apoptosis which binds with $\mathrm{KIT}^{40}$. While we have sought to decipher the functional basis of risk loci based on the cumulative weight of evidence across eQTL, $\mathrm{Hi}-\mathrm{C}$ and ChIP-seq data, a limitation has been reliance on relatively small sample size 
for eQTL analysis. Access to larger eQTL datasets in testicular tissue are likely in the future to address this deficiency enabling a better definition of the causal basis of TGCT risk at each locus.

The 44 risk loci which have now been identified for TGCT collectively account for $34 \%$ of the (fatherto-son) familial risk and hence have potential clinical utility for personalized risk profiling. To assess this potential, we constructed polygenic risk scores (PRS) for TGCT, considering the combined effect of all risk SNPs modelled under a log-normal relative risk distribution. Using this approach the men in the top $1 \%$ of genetic risk have a relative risk of 14 which translates to a $7 \%$ lifetime risk of TGCT (Supplementary Fig. 4).

In summary, we have performed a new TGCT GWAS, identifying 19 new risk loci for TGCT, approximately doubling the number of previously reported SNPs. Using capture $\mathrm{Hi}-\mathrm{C}$ we have generated a chromatin interaction map for TGCT, providing direct physical interactions between non-coding risk SNPs and target gene promoters. Moreover integration of these data together with ChIP-seq chromatin profiling and RNA-seq eQTL analysis, accepting certain caveats, has allowed us to gain preliminary but unbiased tissue-specific insight into the biological basis of TGCT susceptibility. This analysis suggests a model of TGCT susceptibility based on transcriptional dysregulation, which is likely to contribute to the developmental arrest of primordial germ cells coupled with chromosomal instability through defective microtubule function and accompanied upregulation of KIT-MAPK signalling. 


\section{METHODS}

\section{Sample description}

TGCT cases were from the UK $(n=5,992)$ and Scandinavia $(n=1,327)$. The UK cases were ascertained from two studies (1) a UK study of familial testicular cancer and (2) a systematic collection of UK collection of TGCT cases. Case recruitment was via the UK Testicular Cancer Collaboration, a group of oncologists and surgeons treating TGCT in the UK (Supplementary note 1). The studies were coordinated at the Institute of Cancer Research (ICR). Samples and information were obtained with full informed consent and Medical Research and Ethics Committee approval (MREC02/06/66 and 06/MRE06/41). Additional $(n=1,327)$ case samples of Scandinavian origin were used from a previously published GWAS ${ }^{16}$.

Control samples for the primary GWAS were all taken from within the UK. Specifically 2,976 cancerfree, male controls were recruited through two studies within the PRACTICAL Consortium (Supplementary note 2): (1) the UK Genetic Prostate Cancer Study (UKGPCS) (age <65), a study conducted through the Royal Marsden NHS Foundation Trust and (2) SEARCH (Study of Epidemiology \& Risk Factors in Cancer), recruited via GP practices in East Anglia (2003-2009). 4,446 cancer-free female controls from across the UK were recruited via the Breast Cancer Association Consortium (BCAC). Controls from the UK previously published $\mathrm{GWAS}^{11}$ were from two sources within the UK: 2,482 controls were from the 1958 Birth Cohort (1958BC), and 2,587 controls were identified through the UK National Blood Service (NBS) and were genotyped as part of the Wellcome Trust Case Control Consortium. Additional $(n=6,687)$ control samples of Scandinavian origin were used in the meta-analysis, and have been previously described ${ }^{16}$. Control samples for replication genotyping $(n=4,027)$ were taken from two studies, the national study of colorectal cancer genetics (NSCCG) ${ }^{46}$ and GEnetic Lung CAncer Predisposition Study (GELCAPS) ${ }^{47}$. NSCCG and GELCAP controls were spouses of cancer patients with no personal history of cancer at time of ascertainment. 


\section{Primary GWAS}

Genotyping was conducted using a custom Infinium OncoArray-500K BeadChip (Oncoarray) from Illumina (IIlumina, San Diego, CA, USA), comprising a 250K SNP genome-wide backbone and 250K SNP custom content selected across multiple consortia within COGS (Collaborative Oncological Gene-environment Study). Oncoarray genotyping was conducted in accordance with the manufacturer's recommendations by the Edinburgh Clinical Research Facility, Wellcome Trust CRF, Western General Hospital, Edinburgh EH4 2XU.

\section{Published GWAS}

The UK and Scandinavian GWAS have been previously reported ${ }^{8,11,13}$. Briefly the UK GWAS comprised 986 cases genotyped on the Illumina HumanCNV370-Duo bead array (Ilumina, San Diego, CA, USA) and 4,946 controls genotyped on the Illumina Infinium 1.2M array. We analysed data on a common set of 314,861 SNPs successfully genotyped by both arrays. The Scandinavian GWAS ${ }^{16}$, comprised 1,326 cases and 6,687 controls genotyped using the Human OmniExpressExome-8v1 Illumina array.

\section{Quality Control of GWAS}

Oncoarray data was filtered as follows, we excluded individuals with low call rate $(<95 \%)$, with abnormal autosomal heterozygosity or with $>10 \%$ non-European ancestry (based on multidimensional scaling). We filtered out all SNPs with minor allele frequency $<1 \%$, a call rate of $<95 \%$ in cases or controls or with a minor allele frequency of $1-5 \%$ and a call rate of $<99 \%$, and SNPs deviating from Hardy-Weinberg equilibrium $\left(10^{-12}\right.$ in controls and $10^{-5}$ in cases). The final number of SNPs passing quality control filters was 371,504 . Quality control (QC) procedures for the UK and Scandinavian GWAS have been previously described ${ }^{8,11,13,16}$. 


\section{Imputation}

Genome-wide imputation was performed for all GWAS datasets. The 1000 genomes phase 1 data (Sept-13 release) was used as a reference panel, with haplotypes pre-phased using SHAPEIT2 ${ }^{48}$. Imputation was performed using IMPUTE2 software ${ }^{49}$ and association between imputed genotype and TGCT was tested using SNPTEST ${ }^{50}$, under a frequentist model of association. QC was performed on the imputed SNPs; excluding those with INFO score $<0.8$ and MAF $<0.01$.

\section{Replication genotyping}

Replication genotyping of the 37 SNPs was performed by allele-specific KASPar allele-specific SNV primers $^{51}$. Genotyping was conducted by LGC Limited, Unit 1-2 Trident Industrial Estate, Pindar Road, Hoddesdon, UK.

\section{Statistical Analysis}

Study sample size was chosen in order to achieve $>50 \%$ power to detect common variants, defined as MAF $>5 \%$, OR $>1.3^{20}$. For Oncoarray data tests of association between imputed SNPs and TGCT was performed under a probabilistic dosage model in in SNPTESTV2.5 ${ }^{52}$, adjusting for principal components. Inflation in the test statistics was observed at only modest levels, $\lambda_{1000}=1.03$. The inflation factor $\lambda$ was based on the $90 \%$ least-significant SNPs ${ }^{53}$. The adequacy of the case-control matching and possibility of differential genotyping of cases and controls were formally evaluated using Q-Q plots of test statistics (Supplementary Fig. 1). Population ancestry structure for the UK and Scandinavian cohorts was assessed through visualisation of the first two principle components (Supplementary Fig. 5); stable ancestral clustering was observed (Supplementary Table 3). 
Statistical analysis of previously reported GWAS was performed as previously described ${ }^{8,11,13,16,54}$. Meta-analyses were performed using the fixed-effects inverse-variance method based on the $\beta$ estimates and standard errors from each study using META v1.6 ${ }^{55}$. Cochran's Q-statistic to test for heterogeneity and the $\mathrm{I}^{2}$ statistic to quantify the proportion of the total variation due to heterogeneity were calculated ${ }^{56}$. For each new locus we examined evidence of departure from a logadditive (multiplicative) model, to assess any genotype specific effect. Using the Oncoarray data individual genotype data $\mathrm{ORs}$ were calculated for heterozygote $\left(\mathrm{OR}_{\text {het }}\right)$ and homozygote $\left(\mathrm{OR}_{\text {hom }}\right)$ genotypes, which were compared to the per allele ORs. We tested for a difference in these 1 d.f. and 2d.f. logistic regression models to assess for evidence of deviation $(P<0.05)$ from a log-additive model. Using Oncoarray data we examined for statistical interaction between any of the 44 TGCT predisposition loci by evaluating the effect of adding an interaction term to the regression model, adjusted for stage, using a likelihood ratio test (using a significance threshold of $P<2.58 \times 10^{-5}$ to account for 1,936 tests). Regional plots were generated using visPIG software ${ }^{57}$ (Supplementary Fig. 6). Polygenic risk scores (PRS) were constructed using the methodology of Pharoah et $\mathrm{al}^{58}$, based on a log-normal distribution $L N\left(\mu, \sigma^{2}\right)$ with mean $\mu$ and variance $\sigma^{2}$ (i.e. relative risk is normally distributed on a logarithmic scale). The $0.5 \%$ lifetime risk of TGCT risk was based on 2014 UK data ${ }^{59}$, multiplied by relative risk to give lifetime risk per percentile of the PRS. For calculation of the proportion of TGCT genetic risk explained by the 44 loci, a father-to-son relative risk of four was used.

\section{Chromatin mark enrichment analysis}

To examine enrichment in specific ChIP-seq tracks across risk loci we adapted the variant set enrichment method of Cowper-Sal lari et $a^{60}$. Briefly, for each risk locus, a region of strong LD was defined (i.e. $R^{2}>0.8$ and $D^{\prime}>0.8$ ), and SNPs mapping to these regions were termed the associated variant set (AVS). Histone ChIP-seq uniform peak data was obtained from ENCODE ${ }^{21}$ for the NTERA2 
cell line, and data was included for four histone marks. For each of these marks, the overlap of the SNPs in the AVS and the binding sites was determined to produce a mapping tally. A null distribution was produced by randomly selecting SNPs with the same LD structure as the risk associated SNPs, and the null mapping tally calculated. This process was repeated 10,000 times, and approximate $P$ values were calculated as the proportion of permutations where null mapping tally was greater or equal to the AVS mapping tally. An enrichment score was calculated by normalizing the tallies to the median of the null distribution. Thus the enrichment score is the number of standard deviations of the AVS mapping tally from the mean of the null distribution tallies. Tissue specificity was assessed by comparison of enrichment levels in NTERA2, compared to 41 other cell lines from ENCODE ${ }^{21}$, with analysis performed using the same method as above (Supplementary Fig. 2).

\section{Promoter Hi-C}

In situ Hi-C libraries were prepared as described by Rao et al. ${ }^{61}$ with the following modifications: (i) 25 million cells were fixed and processed; (ii) HindIII enzyme (NEB, Ipswich, MA, USA) was used and digestion was performed overnight; (iii) ligation was performed overnight at 16C; (iv) $3 \mu \mathrm{l}$ of $15 \mu \mathrm{M}$ annealed PE adaptors were ligated incubating $3 \mu$ of T4 DNA ligase (NEB, Ipswich, MA, USA) for $2 \mathrm{~h}$ at RT; (vi) 6 cycles of PCR were performed to amplify the libraries before capture. A Sure Select (Agilent, Santa Clara, CA, USA) custom promoter kit was used to perform capture with the same design as described by Misfud et al. ${ }^{62}$. For each capture reaction, $750 \mu \mathrm{g}$ of $\mathrm{Hi}-\mathrm{C}$ libraries were used. Capture was performed following the manufacture protocol and employing a custom reagent kit (Agilent, Santa Clara, CA, USA). Final PCR amplification was performed using 5 cycles to minimise PCR duplicates. 2x100bp sequencing was performed using Illumina HiSeq2000 or 2500 technology

(Illumina, San Diego, CA, USA). The HiCUP pipeline ${ }^{63}$ was used to process raw sequencing reads, map di-tag positions against the reference human genome and remove duplicate reads. The protocol was performed for two independent NTERA2 biological replicates, with cells obtained from the 
laboratory of Prof. Janet Shipley (The Institute of Cancer Research, London) and their identity independently confirmed through STR typing at an external laboratory (Public Health England, Porton Down, UK). Cells were tested and found to be negative for mycoplasma contamination. Both $\mathrm{Hi}-\mathrm{C}$ libraries achieving the following quality control thresholds: $>80 \%$ reads uniquely aligning, $>80 \%$ valid pair rate, $>85 \%$ unique di-tag rate and $>80 \%$ of interactions being cis (Supplementary Table 4 ). Statistically significant interactions were called using the CHiCAGO pipeline ${ }^{64}$, with both biological replicates processed in parallel to obtain a unique list of reproducible NTERA2 contacts. Stability of results across replicates was also verified by processing each sample individually and comparing the significance scores of called interactions; strong correlation was observed between the replicates $(r$ $=0.8, P<5.0 \times 10^{-10}$, Supplementary Fig. 7). Interactions with a $-\log ($ weighted $P$-value $)>5$ were considered significant. To avoid short-range proximity bias interactions of $<40 \mathrm{~kb}$ were excluded. The distribution of interaction distances closely matched the prior published dataset of Misfud et al. $^{62}$ (Supplementary Fig. 8). A Hi-C track plotting read pair counts per Hindlll fragment has been added to region plot figures to demonstrate the underlying signal strength of significant $\mathrm{Hi}-\mathrm{C}$ contacts.

\section{$3 C$ Validation}

$3 \mathrm{C}$ was used to validate selected chromatin interactions detected by $\mathrm{CHi}-\mathrm{C}(3 \mathrm{p} 24.3,4 q 24,11 q 14.1$, 15q22.31, 15q25.2, 16q12.1, and 16q23.1) (Supplementary Fig. 9, Supplementary Table 5). Three replicates of in situ $3 \mathrm{C}$ libraries were prepared using NTERA2 cells. Cell pellets were crosslinked, digested with HindIII, and ligated. Libraries were purified by phenol-chloroform extraction.

For each loci one or more bacterial artificial chromosomes (BACs; Source BioScience, Nottingham, UK) were used as an internal standard (Supplementary Table 6). Clones were streaked and grown before extracting DNA using a QIAGEN Plasmid Maxi Kit (QIAGEN, Hilden, Germany) which was purified by phenol-chloroform extraction. In loci covered by more than one clone, equimolar 
solutions of clones were prepared. Randomly ligated 3 C libraries were generated for each BAC or equimolar solution of BACs.

Unidirectional primer pairs were designed to amplify ligation junctions of the bait and other interacting HindIII fragment (promoter-element, P-E) and around the bait and a flanking control HindIII fragment in between the promoter and distal element (promoter-control, P-C) using Primer $3^{65}$ (Supplementary Tables 7 and 8). Regions were amplified using both P-E and P-C primer pairs in BAC and NTERA2 libraries using a QIAGEN Multiplex PCR Kit (QIAGEN, Hilden, Germany). 5 ng and $100 \mathrm{ng}$ of BAC and NTERA2 library template DNA, respectively, were amplified using the following procedure: initial 15 minute denaturation at $95^{\circ} \mathrm{C}$ followed by 38 cycles of $94^{\circ} \mathrm{C}$ for 0.5 minutes, annealing temperature specific to primer pair for 1.5 minutes seconds, $72^{\circ} \mathrm{C}$ extension for 1.5 minutes, followed by a final 10 minute extension at $72^{\circ} \mathrm{C}$ extension. $5 \mu$ of each PCR reaction was visualised on $2 \%$ agarose gels stained with ethidium bromide. Image ${ }^{66}$ was used to quantify intensities of PCR products and normalise for differential primer efficiency by comparing to equimolar BAC PCR products.

P-E fragments were Sanger sequenced in NTERA2 libraries to confirm fragments visualised on agarose gels as expected (Supplementary Fig. 10).

\section{Chromatin state annotation}

We used ChromHMM ${ }^{67}$ to infer chromatin states by integrating information on histone modifications and DNasel hypersensitivity data to identify combinatorial and spatial patterns of epigenetic marks. Aligned next generation sequencing reads from ChIP-Seq and DNAse-Seq experiments on the NTERA2 cells were downloaded from ENCODE ${ }^{21}$. Read-shift parameters for ChIP-Seq data were calculated using PHANTOMPEAKQUALTOOLS. Genome-wide signal tracks were binarized (including input controls for ChIP-Seq data) and a set of learned models were generated using ChromHMM software ${ }^{67}$. The parameters of the highest scoring model were retained and model states were 
iteratively reduced down from 30 to 5 states. A 27 -state model found to be stable and was subsequently used for segmenting the genome at 200bp resolution (Supplementary Fig. 11).

\section{Expression quantitative trait locus analysis}

We investigated for evidence of association between the SNPs at each locus and tissue specific changes in gene expression using two publically available resources: (i) RNAseq and Affymetrix 6.0 SNP data for 150 TGCT patients from The Cancer Genome Atlas and (ii) normal testicular tissue data from GTEx from 157 samples $^{22}$. Associations between normalized RNA counts per-gene and genotype were quantified using R package 'Matrix eQTL'. Box plots of all eQTL associations are presented in Supplementary Fig. 3 and the tissue in which the association was observed (TGCT or normal testis), along with any other tissues resulting in a positive association, are denoted in Supplementary Table 2. To reduce multiple testing, association tests were only performed between SNP and gene pairs where either: (i) a direct promoter variant was observed (as per column six of Table 2) or (ii) a Hi-C contact to a gene promoter was observed (as per column nine of Table 2), together with functionally active chromatin (as per column seven of Table 2). The SNP used for testing at each locus was selected based on the closest available proxy (highest $R^{2}$ ) to the functional variant (i.e. the promoter or Hi-C contact variant), rather than using the sentinel SNP with the strongest TGCT association. Finally, as a comparison all possible gene/variant eQTL combinations were also tested at each locus (ignoring the functional $\mathrm{Hi}-\mathrm{C} /$ promoter/CHiP-seq data), to provide a reference overview of all possible eQTL associations at each locus (Supplementary Table 9).

\section{Transcription factor binding motif analysis}

The impact of variants on regulatory motifs was assessed for a set of transcription factors (TF) associated with germ cell development. A germ cell specific TF set was utilized, rather than all TF 
globally, to provide increased specificity. An $\mathrm{OMIM}^{68}$ search-term-driven method was used to define the germ cell development TF set, using the following search terms: "germ cell" AND "development" AND "transcription factor" ( $n=46)$. The TF list was then intersected with predicted TF binding motifs based on a library of position weight matrices computed by Kheradpour and Kellis $(2014)^{69} 70$. The intersected dataset contained motif position data for 10 TFs: DMRT1, GATA, KLF4, LHX8, NANOG, POU5F1, PRDM1, SOX2, SOX9, and CTCF. To validate the specificity of these motifs for TGCT we conducted variant set enrichment analysis, using the same method as detailed above (based on Cowper-Sal lari et $a l^{60}$ ), which confirmed enrichment for disruption of these 10 motifs in the 44 TGCT risk loci compared to the null distribution (Supplementary Table 10).

\section{Integration of functional data}

For the integrated functional annotation of risk loci LD blocks were defined as all SNPs in $\mathrm{R}^{2}>0.8$ with the sentinel SNP. Risk loci were then annotated with six types of functional data: (i) presence of a $\mathrm{Hi}-\mathrm{C}$ contact linking to a gene promoter, (ii) presence of an expression quantitative trait locus, (iii) presence of a ChIP-seq peak, (iv) presence of a disrupted transcription factor binding motif, (v) presence of a variant within a gene promoter boundary, with boundaries defined using the Ensembl regulatory build ${ }^{71}$, (vi) presence of a non-synonymous coding change. Candidate causal genes were then assigned to TGCT risk loci using the target genes implicated in annotation tracks (i), (ii), (v) and (vi). Where the data supported multiple gene candidates, the gene with the highest number of individual functional data points was assigned to be the candidate. Where multiple genes have the same number of data points all genes are listed. Competing mechanisms for the same gene (e.g. both coding and promoter variants) were allowed.

\section{ACKNOWLEDGEMENTS}


We thank the subjects with TGCT and the clinicians involved in their care for participation in this study. We thank the patients and all clinicians forming part of the UK Testicular Cancer Collaboration (UKTCC) for their participation in this study. A full list of UKTCC members is included in Supplementary note 1. We acknowledge National Health Service funding to the National Institute for Health Research Biomedical Research Centre. We thank the UK Genetics of Prostate Cancer Study (UKGPCS) study teams for the recruitment of the UKGPCS controls. Genotyping of the OncoArray was funded by the US National Institutes of Health (NIH) [U19 CA 148537 for ELucidating Loci Involved in Prostate cancer SuscEptibility (ELLIPSE) project and X01HG007492 to the Center for Inherited Disease Research (CIDR) under contract number HHSN268201200008I]. Additional analytic support was provided by NIH NCI U01 CA188392 (PI: Schumacher). The PRACTICAL consortium was supported by Cancer Research UK Grants C5047/A7357, C1287/A10118, C1287/A16563, C5047/A3354, C5047/A10692, C16913/A6135, European Commission's Seventh Framework Programme grant agreement $n^{\circ} 223175$ (HEALTH-F2-2009-223175), and The National Institute of Health (NIH) Cancer Post-Cancer GWAS initiative grant: No. 1 U19 CA 148537-01 (the GAME-ON initiative). A full list of PRACTICAL consortium members is included in Supplementary note 2. We would also like to thank the following for funding support: The Institute of Cancer Research and The Everyman Campaign, The Prostate Cancer Research Foundation, Prostate Research Campaign UK (now Prostate Action), The Orchid Cancer Appeal, The National Cancer Research Network UK, The National Cancer Research Institute (NCRI) UK. We are grateful for support of NIHR funding to the NIHR Biomedical Research Centre at The Institute of Cancer Research and The Royal Marsden NHS Foundation Trust. This study would not have been possible without the contributions of the following: M. K. Bolla (BCAC), Q. Wang (BCAC), K. Michailido (BCAC), J. Dennis (BCAC), P. Hall (COGS); D.F. Easton (BCAC), A. Berchuck (OCAC), R. Eeles (PRACTICAL), G. Chenevix-Trench (CIMBA), J. Dennis, P. Pharoah, A. Dunning, K. Muir, J. Peto, A. Lee, and E. Dicks. We also thank the following for their contributions to this project: Jacques Simard, Peter Kraft, Craig Luccarini and the staff of the Centre for Genetic Epidemiology Laboratory; and Kimberly F. Doheny and the staff of the Center for 
Inherited Disease Research (CIDR) genotyping facility. The results published here are in part based upon data generated by the TCGA Research Network: http://cancergenome.nih.gov/. This study makes use of data generated by the Wellcome Trust Case Control Consortium 2 (WTCCC2). A full list of the investigators who contributed to the generation of the data is available from the WTCCC website. We acknowledge the contribution of Elizabeth Rapley and Mike Stratton to the generation of previously published UK GWAS case data. We acknowledge funding from the Swedish Cancer Society (CAN2011/484 and CAN2012/823), the Norwegian Cancer Society (grants number 418975 71081 - PR-2006-0387 and PK01-2007-0375) and the Nordic Cancer Union (grant number S-12/07). This study was supported by the Movember foundation and the Institute of Cancer Research. K. Litchfield is supported by a PhD fellowship from Cancer Research UK. R.S.H. and P.B. are supported by Cancer Research UK (C1298/A8362 Bobby Moore Fund for Cancer Research UK). We thank all the individuals who took part in these studies and all the researchers, clinicians, technicians and administrative staff who have enabled this work to be carried out.

\section{AUTHOR CONTRIBUTIONS}

C.T., K.L., and R.S.H designed the study. Case samples were recruited by A.R., R.H. and through UKTCC. R.E., A.D, K.M, J.P., Z.K-J, N.P. and D.E supplied Oncoarray control data. N.O. administrated genotyping of Oncoarray case samples. D.D. coordinated all case sample administration and tracking. K.L., M.L., A.H. and P.B. prepared samples for genotyping experiments. K.L., M.L., G.O., C.L., K.F. and I.A. conducted all Promotor HiC and $3 \mathrm{C}$ laboratory experiments. Bioinformatics and statistical analyses were designed by C.T., R.S.H and K.L.. K.L., G.M., C.L. and M.L. conducted all Promotor $\mathrm{HiC}$ and $3 \mathrm{C}$ data analysis. K.L. and P.L. conducted transcription factor enrichment analysis. K. L., C.L. and M.L. performed all other bioinformatics and statistical analyses. R.K., T. H., W. K., T.G. and F.W. provided Scandinavian GWAS data. K. L. drafted the manuscript with assistance from C.T., R.S.H., M.L., J.S., J.N. and T.B. All authors reviewed and contributed to the manuscript. 


\section{DATA AVAILABILITY}

Case Oncoarray GWAS data and the Hi-C dataset utilized in this paper have both been deposited in the European Genome-phenome Archive (EGA), which is hosted by the European Bioinformatics Institute (EBI), under the accession codes EGAS00001001836 and EGAS00001001930 respectively.

\section{COMPETING FINANCIAL INTERESTS}

The authors declare no competing financial interests.

\section{FIGURES AND TABLE LEGENDS}

\section{Figure 1 - Study design.}

Figure 2 - Circos plot of integrated functional analysis for all 44 TGCT risk loci. Inner-most ring represents the presence of a $\mathrm{Hi}-\mathrm{C}$ contact in the NTERA2 cell line, the next four rings are narrowpeak histone ChIP-seq tracks for NTERA2, the sixth ring represents - $\log P$ values of TGCT risk association from the Oncoarray GWAS data with green line denoting genome-wide significance and the seventh ring (outer-most) is the functional annotation and classification of candidate causal genes.

Figure 3A-C - Regional plots of three new TGCT loci at A) 8p23.1, B) 15q25.2 and C) 15q22.31. Shown by triangles are the $-\log 10$ association P values of genotyped SNPs, based on Oncoarray data. Shown by circles are imputed SNPs at each locus. The intensity of red shading indicates the strength of LD with the sentinel SNP (labelled). Also shown are the SNP build 37 coordinates in mega-bases, recombination rates in centi-morgans (in light blue) and the genes in the region. Below the gene transcripts are $\mathrm{Hi}-\mathrm{C}$ next generation sequencing read pair counts (gaps represent bait locations) and significant $\mathrm{Hi}-\mathrm{C}$ interactions. Below the axis is a zoomed-in section displaying the surrounding genes for each SNP, the predicted chromHMM states along with an arc depiction of the same $\mathrm{Hi}-\mathrm{C}$ contact(s).

Table 1 - Summary of genotyping results for all genome-wide TGCT risk SNPs ( $n=44)$.

Table 2 - Summary of functional annotation. 


\section{REFERENCES}

1. Manku, G. et al. Changes in the expression profiles of claudins during gonocyte differentiation and in seminomas. Andrology 4, 95-110 (2016).

2. Le Cornet, C. et al. Testicular cancer incidence to rise by $25 \%$ by 2025 in Europe? Modelbased predictions in 40 countries using population-based registry data. Eur J Cancer 50, 8319 (2014).

3. Litchfield, K. et al. Quantifying the heritability of testicular germ cell tumour using both population-based and genomic approaches. Sci Rep 5, 13889 (2015).

4. Swerdlow, A.J., De Stavola, B.L., Swanwick, M.A. \& Maconochie, N.E. Risks of breast and testicular cancers in young adult twins in England and Wales: evidence on prenatal and genetic aetiology. Lancet 350, 1723-8 (1997).

5. McGlynn, K.A., Devesa, S.S., Graubard, B.I. \& Castle, P.E. Increasing incidence of testicular germ cell tumors among black men in the United States. J Clin Oncol 23, 5757-61 (2005).

6. Hemminki, K. \& Li, X. Familial risk in testicular cancer as a clue to a heritable and environmental aetiology. British Journal of Cancer 90, 1765-1770 (2004).

7. Kharazmi, E. et al. Cancer Risk in Relatives of Testicular Cancer Patients by Histology Type and Age at Diagnosis: A Joint Study from Five Nordic Countries. Eur Urol 68, 283-9 (2015).

8. Rapley, E.A. et al. A genome-wide association study of testicular germ cell tumor. Nat Genet 41, 807-10 (2009).

9. Turnbull, C. \& Rahman, N. Genome-wide association studies provide new insights into the genetic basis of testicular germ-cell tumour. Int J Androl 34, e86-96; discussion e96-7 (2011).

10. Kanetsky, P.A. et al. Common variation in KITLG and at 5q31.3 predisposes to testicular germ cell cancer. Nat Genet 41, 811-5 (2009).

11. Turnbull, C. et al. Variants near DMRT1, TERT and ATF7IP are associated with testicular germ cell cancer. Nat Genet 42, 604-7 (2010).

12. Kanetsky, P.A. et al. A second independent locus within DMRT1 is associated with testicular germ cell tumor susceptibility. Hum Mol Genet 20, 3109-17 (2011).

13. Ruark, E. et al. Identification of nine new susceptibility loci for testicular cancer, including variants near DAZL and PRDM14. Nat Genet 45, 686-9 (2013).

14. Bojesen, S.E. et al. Multiple independent variants at the TERT locus are associated with telomere length and risks of breast and ovarian cancer. Nat Genet 45, 371-84, 384e1-2 (2013).

15. Chung, C.C. et al. Meta-analysis identifies four new loci associated with testicular germ cell tumor. Nat Genet 45, 680-5 (2013).

16. Kristiansen, W. et al. Two new loci and gene sets related to sex determination and cancer progression are associated with susceptibility to testicular germ cell tumor. Hum Mol Genet (2015).

17. Litchfield, K. et al. Multi-stage genome-wide association study identifies new susceptibility locus for testicular germ cell tumour on chromosome 3q25. Hum Mol Genet 24, 1169-76 (2015).

18. Litchfield, K. et al. Identification of four new susceptibility loci for testicular germ cell tumour. Nat Commun 6, 8690 (2015).

19. Litchfield, K., Shipley, J. \& Turnbull, C. Common variants identified in genome-wide association studies of testicular germ cell tumour: an update, biological insights and clinical application. Andrology 3, 34-46 (2015).

20. Skol, A.D., Scott, L.J., Abecasis, G.R. \& Boehnke, M. Joint analysis is more efficient than replication-based analysis for two-stage genome-wide association studies (vol 38, pg 209, 2006). Nature Genetics 38, 390-390 (2006).

Running title: Identification of 19 new risk loci for TGCT 
21. Consortium, E.P. et al. An integrated encyclopedia of DNA elements in the human genome. Nature 489, 57-74 (2012).

22. Consortium, G.T. The Genotype-Tissue Expression (GTEx) project. Nat Genet 45, 580-5 (2013).

23. Agnihotri, S. et al. A GATA4-regulated tumor suppressor network represses formation of malignant human astrocytomas. J Exp Med 208, 689-702 (2011).

24. Hellebrekers, D.M. et al. GATA4 and GATA5 are potential tumor suppressors and biomarkers in colorectal cancer. Clin Cancer Res 15, 3990-7 (2009).

25. Tsang, A.P. et al. FOG, a multitype zinc finger protein, acts as a cofactor for transcription factor GATA-1 in erythroid and megakaryocytic differentiation. Cell 90, 109-19 (1997).

26. Adzhubei, I., Jordan, D.M. \& Sunyaev, S.R. Predicting functional effect of human missense mutations using PolyPhen-2. Curr Protoc Hum Genet Chapter 7, Unit7 20 (2013).

27. Ketola, l. et al. Developmental expression and spermatogenic stage specificity of transcription factors GATA-1 and GATA-4 and their cofactors FOG-1 and FOG-2 in the mouse testis. Eur J Endocrinol 147, 397-406 (2002).

28. Zheng, R. \& Blobel, G.A. GATA Transcription Factors and Cancer. Genes Cancer 1, 1178-88 (2010).

29. Kurimoto, K., Yamaji, M., Seki, Y. \& Saitou, M. Specification of the germ cell lineage in mice: a process orchestrated by the PR-domain proteins, Blimp1 and Prdm14. Cell Cycle 7, 3514-8 (2008).

30. Ohinata, Y. et al. A signaling principle for the specification of the germ cell lineage in mice. Cell 137, 571-84 (2009).

31. Yamaji, M. et al. Critical function of Prdm14 for the establishment of the germ cell lineage in mice. Nat Genet 40, 1016-22 (2008).

32. Smith, C.A., McClive, P.J., Western, P.S., Reed, K.J. \& Sinclair, A.H. Conservation of a sexdetermining gene. Nature 402, 601-2 (1999).

33. Rao, S. et al. Differential roles of Sall4 isoforms in embryonic stem cell pluripotency. Mol Cell Biol 30, 5364-80 (2010).

34. Greenbaum, M.P. et al. TEX14 is essential for intercellular bridges and fertility in male mice. Proc Natl Acad Sci U S A 103, 4982-7 (2006).

35. Mondal, G., Ohashi, A., Yang, L., Rowley, M. \& Couch, F.J. Tex14, a Plk1-regulated protein, is required for kinetochore-microtubule attachment and regulation of the spindle assembly checkpoint. Mol Cell 45, 680-95 (2012).

36. Jinks, R.N. et al. Recessive nephrocerebellar syndrome on the Galloway-Mowat syndrome spectrum is caused by homozygous protein-truncating mutations of WDR73. Brain 138, 2173-90 (2015).

37. Colin, E. et al. Loss-of-function mutations in WDR73 are responsible for microcephaly and steroid-resistant nephrotic syndrome: Galloway-Mowat syndrome. Am J Hum Genet 95, 63748 (2014).

38. Petrovic, A. et al. The MIS12 complex is a protein interaction hub for outer kinetochore assembly. J Cell Biol 190, 835-52 (2010).

39. Rao, C.V., Yamada, H.Y., Yao, Y. \& Dai, W. Enhanced genomic instabilities caused by deregulated microtubule dynamics and chromosome segregation: a perspective from genetic studies in mice. Carcinogenesis 30, 1469-74 (2009).

40. Barisic, M. et al. Mitosis. Microtubule detyrosination guides chromosomes during mitosis. Science 348, 799-803 (2015).

41. Ma, W. \& Viveiros, M.M. Depletion of pericentrin in mouse oocytes disrupts microtubule organizing center function and meiotic spindle organization. Mol Reprod Dev 81, 1019-29 (2014).

Running title: Identification of 19 new risk loci for TGCT 
42. Litchfield, K. et al. Whole-exome sequencing reveals the mutational spectrum of testicular germ cell tumours. Nat Commun 6, 5973 (2015).

43. Zeron-Medina, J. et al. A polymorphic p53 response element in KIT ligand influences cancer risk and has undergone natural selection. Cell 155, 410-22 (2013).

44. De Miguel, M.P., Cheng, L., Holland, E.C., Federspiel, M.J. \& Donovan, P.J. Dissection of the cKit signaling pathway in mouse primordial germ cells by retroviral-mediated gene transfer. Proc Natl Acad Sci U S A 99, 10458-63 (2002).

45. Yu, M. et al. The scaffolding adapter Gab2, via Shp-2, regulates kit-evoked mast cell proliferation by activating the Rac/JNK pathway. J Biol Chem 281, 28615-26 (2006).

46. Penegar, S. et al. National study of colorectal cancer genetics. Br J Cancer 97, 1305-9 (2007).

47. Eisen, T., Matakidou, A., Houlston, R. \& Consortium, G. Identification of low penetrance alleles for lung cancer: the GEnetic Lung CAncer Predisposition Study (GELCAPS). BMC Cancer 8, 244 (2008).

48. Delaneau, O., Marchini, J. \& Zagury, J.F. A linear complexity phasing method for thousands of genomes. Nat Methods 9, 179-81 (2012).

49. Howie, B., Fuchsberger, C., Stephens, M., Marchini, J. \& Abecasis, G.R. Fast and accurate genotype imputation in genome-wide association studies through pre-phasing. Nat Genet 44, 955-9 (2012).

50. Marchini, J. \& Howie, B. Genotype imputation for genome-wide association studies. Nat Rev Genet 11, 499-511 (2010).

51. Cuppen, E. Genotyping by Allele-Specific Amplification (KASPar). CSH Protoc 2007, pdb prot4841 (2007).

52. Marchini, J., Howie, B., Myers, S., McVean, G. \& Donnelly, P. A new multipoint method for genome-wide association studies by imputation of genotypes. Nat Genet 39, 906-13 (2007).

53. Clayton, D.G. et al. Population structure, differential bias and genomic control in a largescale, case-control association study. Nat Genet 37, 1243-6 (2005).

54. Litchfield, K. et al. Multi-stage genome wide association study identifies new susceptibility locus for testicular germ cell tumour on chromosome 3q25. Hum Mol Genet (2014).

55. Liu, J.Z. et al. Meta-analysis and imputation refines the association of $15 q 25$ with smoking quantity. Nat Genet 42, 436-40 (2010).

56. Higgins, J.P. \& Thompson, S.G. Quantifying heterogeneity in a meta-analysis. Stat Med 21, 1539-58 (2002).

57. Scales, M., Jager, R., Migliorini, G., Houlston, R.S. \& Henrion, M.Y. visPIG--a web tool for producing multi-region, multi-track, multi-scale plots of genetic data. PLoS One 9, e107497 (2014).

58. Pharoah, P.D.P. et al. Polygenic susceptibility to breast cancer and implications for prevention. Nature Genetics 31, 33-36 (2002).

59. CRUK. (2014).

60. Cowper-Sal lari, R. et al. Breast cancer risk-associated SNPs modulate the affinity of chromatin for FOXA1 and alter gene expression. Nat Genet 44, 1191-8 (2012).

61. Rao, S.S. et al. A 3D map of the human genome at kilobase resolution reveals principles of chromatin looping. Cell 159, 1665-80 (2014).

62. Mifsud, B. et al. Mapping long-range promoter contacts in human cells with high-resolution capture Hi-C. Nat Genet 47, 598-606 (2015).

63. Wingett, S. et al. HiCUP: pipeline for mapping and processing Hi-C data. F100ORes 4, 1310 (2015).

64. Jonathan Cairns, P.F.-P., Steven W. Wingett, Csilla Várnai, Andrew Dimond, Vincent Plagnol, Daniel Zerbino, Stefan Schoenfelder, Biola-Maria Javierre, Cameron Osborne, Peter Fraser,

Running title: Identification of 19 new risk loci for TGCT 
Mikhail Spivakov. CHiCAGO: Robust Detection of DNA Looping Interactions in Capture Hi-C data. BioRxiv (2016).

65. Untergasser, A. et al. Primer3--new capabilities and interfaces. Nucleic Acids Res 40, e115 (2012).

66. Schneider, C.A., Rasband, W.S. \& Eliceiri, K.W. NIH Image to ImageJ: 25 years of image analysis. Nat Methods 9, 671-5 (2012).

67. Ernst, J. \& Kellis, M. ChromHMM: automating chromatin-state discovery and characterization. Nat Methods 9, 215-6 (2012).

68. Hamosh, A., Scott, A.F., Amberger, J.S., Bocchini, C.A. \& McKusick, V.A. Online Mendelian Inheritance in Man (OMIM), a knowledgebase of human genes and genetic disorders. Nucleic Acids Res 33, D514-7 (2005).

69. Ward, L.D. \& Kellis, M. HaploReg: a resource for exploring chromatin states, conservation, and regulatory motif alterations within sets of genetically linked variants. Nucleic Acids Res 40, D930-4 (2012).

70. Kheradpour, P. \& Kellis, M. Systematic discovery and characterization of regulatory motifs in ENCODE TF binding experiments. Nucleic Acids Res 42, 2976-87 (2014).

71. Zerbino, D.R., Wilder, S.P., Johnson, N., Juettemann, T. \& Flicek, P.R. The ensembl regulatory build. Genome Biol 16, 56 (2015).

Running title: Identification of 19 new risk loci for TGCT 


\section{Supplementary Data for:}

Identification of 19 new risk loci reveals gene regulatory mechanisms determining susceptibility to testicular germ cell tumour

Kevin Litchfield ${ }^{1}$, Max Levy ${ }^{1}$, Giulia Orlando ${ }^{1}$, Chey Loveday ${ }^{1}$, Philip Law ${ }^{1}$, Gabriele Migliorini ${ }^{1}$, Amy Holroyd ${ }^{1}$, Peter Broderick ${ }^{1}$, Robert Karlsson ${ }^{2}$, Trine B Haugen ${ }^{3}$, Wenche Kristiansen ${ }^{3}$, Jérémie Nsengimana ${ }^{4}$, Kerry Fenwick ${ }^{5}$, Ioannis Assiotis ${ }^{5}$, ZSofia Kote-Jarai ${ }^{1}$, Alison M. Dunning ${ }^{6}$, Kenneth Muir $^{8,9}$, Julian Peto ${ }^{10}$, Rosalind Eeles ${ }^{1,11}$, Douglas F Easton ${ }^{6,7}$, Darshna Dudakia ${ }^{1}$, Nick Orr ${ }^{12}$, Nora Pashayan $^{13}$, UK Testicular Cancer Collaboration*, the PRACTICAL consortium*, D. Timothy Bishop ${ }^{4}$, Alison Reid ${ }^{14}$, Robert A Huddart ${ }^{14}$, Janet Shipley ${ }^{15}$, Tom Grotmol $^{16}$, Fredrik Wiklund ${ }^{2}$, Richard S Houlston $^{1}$, Clare Turnbull ${ }^{1,17}$

* See supplementary notes 1 and 2

\section{Supplementary Tables:}

Supplementary Table 1: Histone enrichment analysis Supplementary Table 2: eQTL associations by tissue type Supplementary Table 3: Allele frequencies by study Supplementary Table 4: Hi-C quality control metrics Supplementary Table 5: 3C PCR raw densitometry values Supplementary Table 6: Bacterial artificial chromosomes Supplementary Table 7: PCR primers used to amplify 3 C promoter-control interactions Supplementary Table 8: PCR primers used to amplify 3 C promoter-element interactions Supplementary Table 9: All eQTL associations Supplementary Table 10: Transcription factor binding motif enrichment analysis

\section{Supplementary Figures:}

Supplementary Figure 1: Quantile-Quantile plot

Supplementary Figure 2: Evidence of tissue specific histone mark enrichment

Supplementary Figure 3: eQTL association boxplots

Supplementary Figure 4: Polygenic risk score model

Supplementary Figure 5: Principle component analysis plot of ethnicity structure 
Supplementary Figure 6: Regional plots

Supplementary Figure 7: $\mathrm{Hi}-\mathrm{C}$ interaction scores for biological replicates one and two

Supplementary Figure 8: Density plot showing distribution of $\mathrm{Hi}-\mathrm{C}$ interaction distances

Supplementary Figure 9: Validation of Hi-C data by 3C PCR assay

Supplementary Figure 10: Sanger sequencing chromatograms of 3C PCR products

Supplementary Figure 11: CHROMHMM emission parameters 
Supplementary Table1 . Histone mark enrichment

\begin{tabular}{|l|rc|}
\hline \multirow{2}{*}{ Histone Mark } & \multicolumn{2}{|c|}{$\begin{array}{c}\text { NTERA2 } \\
\text { (TGCT cells) }\end{array}$} \\
\cline { 2 - 3 } & Fold-Enrichment & $P$-value \\
\hline H3k4me3 & 8.6 & $1.0 \mathrm{E}-04$ \\
H3k9ac & 8.1 & $1.3 \mathrm{E}-04$ \\
H3k4me1 & 5.4 & $4.0 \mathrm{E}-03$ \\
H3k9me3 & 2.2 & $7.9 \mathrm{E}-02$ \\
\hline
\end{tabular}


Supplementary Table2 - eQTL associations by tissue type.

\begin{tabular}{|c|c|c|c|c|c|c|c|}
\hline \multirow[b]{2}{*}{$\begin{array}{l}\text { Sentinal SNP (strongest } \\
\text { association with TGCT) }\end{array}$} & \multirow[b]{2}{*}{$\begin{array}{l}\text { eQTL SNP } \\
\text { (strongest eQTL } \\
\text { assocation at locus) } \\
\end{array}$} & \multirow[b]{2}{*}{$\mathbf{R}^{2}$} & \multirow[b]{2}{*}{ Cyto-band } & \multirow[b]{2}{*}{ Gene } & \multicolumn{3}{|c|}{ eQTL association signal by tissue type: } \\
\hline & & & & & Normal Testicular Tissue & TGC Tumor Tissue & Other tissues \\
\hline rs2072499 & rs1052067 & 0.56 & $1 q 22$ & ССТЗ ${ }^{1}$ & $P<5 \times 10^{-4}$ & - & - \\
\hline rs17021463 & rs2865350 & 0.96 & $4 q 22.3$ & SMARCAD $1^{2}$ & - & $P<5 \times 10^{-2}$ & - \\
\hline rs2720460 & rs2720460 & 1.00 & $4 q 24$ & MANBA $^{2}$ & - & $P<5 \times 10^{-2}$ & - \\
\hline rs210138 & rs210138 & 1.00 & $6 p 21.31$ & $B A K 1^{2}$ & - & $P<5 \times 10^{-2}$ & $\begin{array}{c}\quad P<5 \times 10^{-4} \text { : Muscle - Skeletal, } \\
\text { Whole Blood, Lung, Artery - Aorta. }\end{array}$ \\
\hline rs17153755 & rs1004712 & 0.68 & $8 p 23.1$ & GATA4 $^{2}$ & - & $P<5 \times 10^{-2}$ & Non-significant \\
\hline rs1009647 & rs1538257 & 0.59 & $14 q 22.3$ & ATG14 & $P<5 \times 10^{-4}$ & - & $\begin{array}{c}P<5 \times 10^{-4} \text { : Esophagus - Mucosa, } \\
\text { Skin, Artery - Tibial. }\end{array}$ \\
\hline rs11071896 & rs11629783 & 0.86 & $15 q 22.31$ & SNAPC5 ${ }^{2}$ & - & $P<5 \times 10^{-2}$ & - \\
\hline rs56046484 & rs2304416 & 0.99 & $15 q 25.2$ & $W D R 73^{2}$ & - & $P<5 \times 10^{-2}$ & - \\
\hline rs4561483 & rs2075158 & 0.84 & $16 p 13.13$ & GSPT1 $^{3}$ & \multicolumn{3}{|c|}{ Previously published } \\
\hline rs8046148 & rs12930079 & 0.54 & $16 q 12.1$ & HEATR $^{1}$ & $P<5 \times 10^{-4}$ & - & $\begin{array}{c}P<5 \times 10^{-4}: 20+\text { normal tissue } \\
\text { types. }\end{array}$ \\
\hline rs4888262 & rs58136167 & 0.51 & $16 q 23.1$ & RFWD $3^{1}$ & $P<5 \times 10^{-4}$ & - & $\begin{array}{c}P<5 \times 10^{-4}: 10+\text { normal tissue } \\
\text { types. }\end{array}$ \\
\hline rs9905704 & rs654778 & 0.32 & $17 q 22$ & TEX14 ${ }^{1}$ & $P<5 \times 10^{-4}$ & - & $\begin{array}{c}P<5 \times 10^{-4} \text { : Esophagus - } \\
\text { Muscularis, Skin, Thyroid, Nerve - } \\
\text { Tibial. }\end{array}$ \\
\hline
\end{tabular}

${ }^{1}$ Signficant vs threshold corrected for 96 multiple tests

${ }^{2}$ Nominally significant at $P<0.05$

${ }^{3}$ eQTL identified in previous study 
Supplementary Table 3 - Summary of allele frequencies across GWAS datasets for all genome-wide TGCT risk SNPs ( $n=44)$. New loci $(n=19)$ discovered through this study are marked in bold.

\begin{tabular}{|c|c|c|c|c|c|c|c|c|c|}
\hline \multirow[b]{2}{*}{$\mathrm{SNP}^{1}$} & \multirow[b]{2}{*}{ Chr. } & \multirow[b]{2}{*}{ bp (b37) } & \multirow[b]{2}{*}{$\begin{array}{c}\text { Alleles } \\
\text { (A/B) }\end{array}$} & \multicolumn{2}{|c|}{ UK - Oncoarray } & \multicolumn{2}{|c|}{ UK - Published GWAS } & \multicolumn{2}{|c|}{$\begin{array}{c}\text { Scandinavian - Published } \\
\text { GWAS }\end{array}$} \\
\hline & & & & $\begin{array}{c}\text { Allele B } \\
\text { Frequency - } \\
\text { Cases }\end{array}$ & $\begin{array}{c}\text { Allele B } \\
\text { Frequency - } \\
\text { Controls }\end{array}$ & $\begin{array}{c}\text { Allele B } \\
\text { Frequency - } \\
\text { Cases }\end{array}$ & $\begin{array}{c}\text { Allele B } \\
\text { Frequency - } \\
\text { Controls }\end{array}$ & $\begin{array}{c}\text { Allele B } \\
\text { Frequency - } \\
\text { Cases }\end{array}$ & $\begin{array}{c}\text { Allele B } \\
\text { Frequency - } \\
\text { Controls }\end{array}$ \\
\hline rs4240895 & 1 & 9713386 & $\mathrm{C} / \mathrm{T}$ & 0.41 & 0.38 & 0.40 & 0.38 & 0.41 & 0.40 \\
\hline rs2072499 & 1 & 156169610 & $A / G$ & 0.39 & 0.36 & 0.40 & 0.35 & 0.40 & 0.35 \\
\hline rs3790672 & 1 & 165873392 & $\mathrm{~T} / \mathrm{C}$ & 0.32 & 0.29 & 0.33 & 0.28 & 0.35 & 0.28 \\
\hline rs7581030 & 2 & 71572455 & $\mathrm{C} / \mathrm{T}$ & 0.26 & 0.24 & 0.26 & 0.23 & 0.27 & 0.24 \\
\hline rs10510452 & 3 & 16625048 & $\mathrm{~A} / \mathrm{G}$ & 0.28 & 0.31 & 0.27 & 0.32 & 0.23 & 0.27 \\
\hline rs11705932 & 3 & 141818850 & $\mathrm{C} / \mathrm{T}$ & 0.18 & 0.20 & 0.18 & 0.21 & 0.20 & 0.21 \\
\hline rs1510272 & 3 & 156300724 & $\mathrm{C} / \mathrm{T}$ & 0.23 & 0.26 & 0.22 & 0.27 & 0.22 & 0.27 \\
\hline rs6821144 & 4 & 76520651 & G/A & 0.09 & 0.11 & 0.09 & 0.11 & 0.10 & 0.11 \\
\hline rs17021463 & 4 & 95224812 & $\mathrm{~T} / \mathrm{G}$ & 0.45 & 0.42 & 0.46 & 0.42 & 0.44 & 0.42 \\
\hline rs2720460 & 4 & 104054686 & $A / G$ & 0.33 & 0.38 & 0.33 & 0.39 & 0.34 & 0.41 \\
\hline rs4862848 & 4 & 188921440 & $\mathbf{A} / \mathbf{G}$ & 0.38 & 0.35 & 0.38 & 0.34 & 0.37 & 0.32 \\
\hline rs2736100 & 5 & 1286516 & $C / A$ & 0.45 & 0.50 & 0.43 & 0.51 & 0.44 & 0.50 \\
\hline rs3805663 & 5 & 134342720 & $C / A$ & 0.35 & 0.37 & 0.33 & 0.38 & 0.33 & 0.35 \\
\hline rs4624820 & 5 & 141681788 & $\mathrm{G} / \mathrm{A}$ & 0.37 & 0.46 & 0.37 & 0.46 & 0.40 & 0.49 \\
\hline rs210138 & 6 & 33542538 & $A / G$ & 0.25 & 0.19 & 0.27 & 0.19 & 0.25 & 0.18 \\
\hline rs11155671 & 6 & 149972132 & $\mathbf{G} / \mathbf{A}$ & 0.31 & 0.35 & 0.32 & 0.34 & 0.33 & 0.36 \\
\hline rs12699477 & 7 & 1968953 & $\mathrm{~T} / \mathrm{C}$ & 0.42 & 0.37 & 0.42 & 0.38 & 0.38 & 0.35 \\
\hline rs17689040 & 7 & 40920313 & $\mathbf{C} / \mathbf{G}$ & 0.46 & 0.42 & 0.44 & 0.42 & 0.44 & 0.40 \\
\hline rs17153755 & 8 & 11611500 & $\mathbf{C} / \mathbf{G}$ & 0.32 & 0.36 & 0.34 & 0.35 & 0.36 & 0.40 \\
\hline rs7010162 & 8 & 70976505 & $\mathrm{C} / \mathrm{T}$ & 0.35 & 0.38 & 0.35 & 0.39 & 0.37 & 0.41 \\
\hline rs7040024 & 9 & 845516 & $\mathrm{~A} / \mathrm{C}$ & 0.18 & 0.25 & 0.16 & 0.25 & 0.19 & 0.25 \\
\hline rs7107174 & 11 & 77996403 & $C / A$ & 0.18 & 0.16 & 0.17 & 0.15 & 0.19 & 0.17 \\
\hline rs648090 & 11 & 125071163 & $\mathbf{A} / \mathbf{G}$ & 0.27 & 0.30 & 0.27 & 0.29 & 0.28 & 0.31 \\
\hline rs2900333 & 12 & 14653867 & $\mathrm{C} / \mathrm{T}$ & 0.34 & 0.38 & 0.32 & 0.38 & 0.35 & 0.37 \\
\hline rs4931000 & 12 & 32141495 & $\mathbf{A} / \mathbf{G}$ & 0.24 & 0.22 & 0.24 & 0.22 & 0.22 & 0.19 \\
\hline rs7315956 & 12 & 70563865 & $\mathbf{A} / \mathbf{G}$ & 0.35 & 0.33 & 0.36 & 0.33 & 0.33 & 0.30 \\
\hline rs3782181 & 12 & 88953561 & $C / A$ & 0.10 & 0.22 & 0.11 & 0.22 & 0.07 & 0.17 \\
\hline rs1009647 & 14 & 55880047 & G/A & 0.25 & 0.27 & 0.24 & 0.28 & 0.26 & 0.27 \\
\hline rs11071896 & 15 & 66821250 & $\mathbf{A} / \mathbf{G}$ & 0.28 & 0.25 & 0.29 & 0.25 & 0.27 & 0.25 \\
\hline rs56046484 & 15 & 85605427 & $\mathbf{G} / \mathbf{T}$ & 0.18 & 0.21 & 0.18 & 0.21 & 0.21 & 0.22 \\
\hline rs4561483 & 16 & 11920037 & $A / G$ & 0.37 & 0.35 & 0.38 & 0.34 & 0.34 & 0.33 \\
\hline rs7404843 & 16 & 15530708 & $\mathrm{~T} / \mathrm{G}$ & 0.13 & 0.11 & 0.14 & 0.11 & 0.15 & 0.12 \\
\hline rs8046148 & 16 & 50142944 & $A / G$ & 0.20 & 0.21 & 0.18 & 0.22 & 0.17 & 0.19 \\
\hline rs4888262 & 16 & 74670458 & $\mathrm{C} / \mathrm{T}$ & 0.47 & 0.50 & 0.47 & 0.51 & 0.46 & 0.51 \\
\hline rs55637647 & 16 & 88549264 & $\mathrm{C} / \mathrm{G}$ & 0.41 & 0.38 & 0.42 & 0.37 & 0.37 & 0.35 \\
\hline rs7501939 & 17 & 36101156 & $\mathrm{~T} / \mathrm{C}$ & 0.35 & 0.40 & 0.35 & 0.41 & 0.33 & 0.38 \\
\hline rs9905704 & 17 & 56632543 & $\mathrm{G} / \mathrm{T}$ & 0.27 & 0.33 & 0.28 & 0.33 & 0.26 & 0.29 \\
\hline rs9966612 & 18 & 649311 & A/G & 0.36 & 0.34 & 0.31 & 0.28 & 0.31 & 0.30 \\
\hline rs2195987 & 19 & 24149545 & $\mathrm{C} / \mathrm{T}$ & 0.12 & 0.13 & 0.20 & 0.23 & 0.18 & 0.23 \\
\hline rs2241024 & 19 & 28257393 & G/A & 0.18 & 0.22 & 0.18 & 0.20 & 0.17 & 0.21 \\
\hline rs4599029 & 19 & 54284689 & G/T & 0.24 & 0.27 & 0.25 & 0.26 & 0.26 & 0.28 \\
\hline rs12481572 & 20 & 50708054 & $A / T$ & 0.21 & 0.18 & 0.22 & 0.20 & 0.20 & 0.18 \\
\hline rs2839186 & 21 & 47690068 & $\mathrm{C} / \mathrm{T}$ & 0.50 & 0.46 & 0.52 & 0.47 & 0.48 & 0.44 \\
\hline rs739525 & 22 & 21332441 & $\mathrm{~T} / \mathrm{C}$ & 0.45 & 0.48 & 0.44 & 0.47 & 0.44 & 0.46 \\
\hline
\end{tabular}


Supplementary table4 : NGS metrics for CHiC libraries. The table reports HiC libraries metrics obtained using HICUP pipeline.

\begin{tabular}{|l|c|c|c|c|c|c|}
\hline & $\begin{array}{c}\text { Total Reads } \\
\text { Processed }\end{array}$ & Truncated & \%Truncated & Not truncated & $\begin{array}{c}\text { Average length } \\
\text { truncated sequence }\end{array}$ \\
\hline NTERA1_CHiC_replicate1_R1 & $1,101,182,035$ & $231,325,197$ & 21.0 & $869,856,838$ & 79.0 & 57.23 \\
\hline NTERA1_CHiC_replicate1_R2 & $1,101,182,035$ & $226,357,230$ & 20.6 & $874,824,805$ & 79.4 & 56.47 \\
\hline NTERA1_CHiC_replicate2_R1 & $539,696,994$ & $108,255,974$ & 20.1 & $431,441,020$ & 79.9 & 57.4 \\
\hline NTERA1_CHiC_replicate2_R2 & $539,696,994$ & $102,950,769$ & 19.1 & $436,746,225$ & 80.9 & 56.7 \\
\hline
\end{tabular}

\begin{tabular}{|c|c|c|c|c|c|c|c|c|c|c|c|}
\hline & $\begin{array}{l}\text { Total reads } \\
\text { processed }\end{array}$ & $\begin{array}{c}\text { Reads too } \\
\text { short to map }\end{array}$ & $\begin{array}{c}\% \text { Reads too short } \\
\text { to map }\end{array}$ & $\begin{array}{c}\text { Unique } \\
\text { alignments }\end{array}$ & $\%$ Unique alignments & Multiple alignments & $\begin{array}{l}\text { \%Multiple } \\
\text { alignments }\end{array}$ & Failed to align & $\begin{array}{l}\text { \%failed to } \\
\text { align }\end{array}$ & Paired & \%Paired \\
\hline \begin{tabular}{|l} 
NTERA1_CHiC_replicate1_R1 \\
\end{tabular} & $1,101,182,035$ & $10,849,893$ & 1.0 & $912,392,203$ & 82.9 & $85,028,672$ & 7.7 & $92,911,267$ & 8.4 & $760,465,317$ & 69.1 \\
\hline NTERA1_CHiC_replicate1_R2 & $1,101,182,035$ & $10,961,364$ & 1.0 & $899,439,287$ & 81.7 & $85,840,243$ & 7.8 & $104,941,141$ & 9.5 & $760,465,317$ & 69.1 \\
\hline NTERA1_CHiC_replicate2_R1 & $539,696,994$ & $5,248,668$ & 1.0 & $465,981,695$ & 86.3 & $35,827,878$ & 6.6 & $32,638,753$ & 6.0 & $401,330,333$ & 74.4 \\
\hline NTERA1_CHiC_replicate2_R2 & $539,696,994$ & $5,155,081$ & 1.0 & $457,854,578$ & 84.8 & $36,334,316$ & 6.7 & $40,353,019$ & 7.5 & $401,330,333$ & 74.4 \\
\hline
\end{tabular}

\begin{tabular}{|c|c|c|c|c|c|c|c|c|}
\hline & Total pairs & Valid pairs & $\begin{array}{c}\text { Same } \\
\text { circularised }\end{array}$ & $\begin{array}{c}\text { Same dangling } \\
\text { ends }\end{array}$ & Same internal & Re-ligation & $\begin{array}{l}\text { Contiguous } \\
\text { sequence }\end{array}$ & Wrong size \\
\hline \begin{tabular}{|l|} 
NTERA2_CHiC_replicate1 \\
\end{tabular} & $760,465,317$ & $628,903,392$ & $6,246,866$ & $3,049,526$ & $30,787,233$ & $22,863,978$ & $1,958,803$ & $66,655,519$ \\
\hline \begin{tabular}{|l|} 
NTERA2_CHiC_replicate2 \\
\end{tabular} & $401,330,333$ & $334,615,620$ & $3,399,000$ & $1,310,086$ & $21,603,379$ & $16,718,749$ & $1,332,132$ & $22,351,367$ \\
\hline
\end{tabular}

\begin{tabular}{|c|c|c|c|c|c|c|c|c|}
\hline & Total pairs & Valid pairs & $\begin{array}{c}\text { Same } \\
\text { circularised }\end{array}$ & $\begin{array}{c}\text { Same dangling } \\
\text { ends }\end{array}$ & Same internal & Re-ligation & $\begin{array}{l}\text { Contiguous } \\
\text { sequence }\end{array}$ & Wrong size \\
\hline NTERA2_CHiC_replicate1 & $760,465,317$ & 82.7 & 0.8 & 0.4 & 4.0 & 3.0 & 0.3 & 8.8 \\
\hline NTERA2_CHiC_replicate2 & $401,330,333$ & 83.4 & 0.8 & 0.3 & 5.4 & 4.2 & 0.3 & 5.6 \\
\hline
\end{tabular}

\begin{tabular}{|l|r|r|r|r|r|}
\hline & $\begin{array}{c}\text { Read pairs } \\
\text { processed }\end{array}$ & Unique di-tags & $\begin{array}{c}\text { Cis <10kbp of } \\
\text { uniques }\end{array}$ & $\begin{array}{c}\text { Cis }>10 \mathrm{kbp} \text { of } \\
\text { uniques }\end{array}$ & \multicolumn{1}{c|}{ Trans of uniques } \\
\hline NTERA2_CHiC_replicate1 & $628,903,392$ & $534,979,228$ & $56,954,249$ & $374,778,991$ & $103,245,988$ \\
\hline NTERA2_CHiC_replicate2 & $334,615,620$ & $289,198,027$ & $32,454,613$ & $202,713,861$ & $54,029,553$ \\
\hline
\end{tabular}

\begin{tabular}{|l|c|r|c|c|c|}
\hline & $\begin{array}{c}\text { Read pairs } \\
\text { processed }\end{array}$ & Unique di-tags & $\begin{array}{c}\text { Cis <10kbp of } \\
\text { uniques }\end{array}$ & $\begin{array}{c}\text { Cis >10kbp of } \\
\text { uniques }\end{array}$ & Trans of uniques \\
\hline NTERA2_CHiC_replicate1 & $268,657,417$ & 85.1 & 10.6 & 70.1 & 19.3 \\
\hline NTERA2_CHiC_replicate2 & $313,873,816$ & 86.4 & 11.2 & 70.1 & 18.7 \\
\hline
\end{tabular}


Supplementary Table 5. 3C PCR raw densitometry values.

\begin{tabular}{|c|c|c|c|c|c|c|c|c|}
\hline \multirow[b]{2}{*}{ Region } & \multirow[b]{2}{*}{ Gene } & \multirow[b]{2}{*}{ Library } & \multicolumn{3}{|c|}{ Promoter-Element } & \multicolumn{3}{|c|}{ Promoter-Control } \\
\hline & & & Area & $\begin{array}{l}\text { Ave } \\
\text { RIF }\end{array}$ & SD & Area & $\begin{array}{l}\text { Ave } \\
\text { RIF }\end{array}$ & SD \\
\hline \multirow{4}{*}{ 3p24.3 } & \multirow{4}{*}{ OXNAD1 } & BAC & 28912 & \multirow{4}{*}{0.6} & \multirow{4}{*}{0.04} & 26922 & \multirow{4}{*}{0.3} & \multirow{4}{*}{0.06} \\
\hline & & NTERA 3C 1 & 17588 & & & 9514 & & \\
\hline & & NTERA 3C 2 & 18555 & & & 8319 & & \\
\hline & & NTERA 3C 3 & 16175 & & & 6337 & & \\
\hline \multirow{4}{*}{$4 q 24$} & \multirow{4}{*}{ MANBA } & BAC & 11332 & \multirow{4}{*}{0.4} & \multirow{4}{*}{0.14} & 12847 & \multirow{4}{*}{0.1} & \multirow{4}{*}{0.11} \\
\hline & & NTERA 3C 1 & 2817 & & & 801 & & \\
\hline & & NTERA 3C 2 & 3922 & & & 0 & & \\
\hline & & NTERA 3C 3 & 5986 & & & 2850 & & \\
\hline \multirow{4}{*}{$11 q 14.1$} & \multirow{4}{*}{ GAB2 } & BAC & 18938 & \multirow{4}{*}{0.5} & \multirow{4}{*}{0.05} & 20513 & \multirow{4}{*}{0.3} & \multirow{4}{*}{0.02} \\
\hline & & NTERA 3C 1 & 7931 & & & 5114 & & \\
\hline & & NTERA 3C 2 & 8181 & & & 5693 & & \\
\hline & & NTERA 3C 3 & 9834 & & & 4996 & & \\
\hline \multirow{4}{*}{$15 q 22.31$} & \multirow{4}{*}{ MAP2K1 } & BAC & 13082 & \multirow{4}{*}{1.0} & \multirow{4}{*}{0.27} & 18873 & \multirow{4}{*}{0.4} & \multirow{4}{*}{0.10} \\
\hline & & NTERA 3C 1 & 11778 & & & 6703 & & \\
\hline & & NTERA 3C 2 & 9764 & & & 3794 & & \\
\hline & & NTERA 3C 3 & 16525 & & & 10980 & & \\
\hline \multirow{4}{*}{$15 q 25.2$} & & BAC & 10260 & & & 8498 & & \\
\hline & & NTERA 3C 1 & 3086 & & & 1459 & & \\
\hline & WDR/3 & NTERA 3C 2 & 2077 & 0.3 & 0.05 & 940 & 0.1 & 0.03 \\
\hline & & NTERA 3C 3 & 2623 & & & 1217 & & \\
\hline & & BAC & 18000 & & & 12470 & & \\
\hline 160121 & HFATR3 & NTERA 3C 1 & 12946 & 08 & 0.5 & 2843 & 01 & 011 \\
\hline 10912.1 & HEAIRO & NTERA 3C 2 & 14069 & 0.8 & 0.05 & 5914 & 0.4 & 0.14 \\
\hline & & NTERA 3C 3 & 14747 & & & 5786 & & \\
\hline & & BAC & 16783 & & & 18294 & & \\
\hline 160231 & RFWIח3 & NTERA 3C 1 & 12543 & 08 & 004 & 4654 & 04 & 016 \\
\hline $16 q 23.1$ & RFVVD3 & NTERA 3C 2 & 13042 & 0.8 & 0.04 & 8216 & 0.4 & 0.16 \\
\hline & & NTERA 3C 3 & 13857 & & & 10492 & & \\
\hline
\end{tabular}

$R I F$, relative interaction frequency; area, area under the graph; $S D$, standard deviation. 
Supplementary Table 6. Bacterial artificial chromosomes (BACs) from the RPCI human BAC library 11 (RP11) used in $3 \mathrm{C}$ validation of selected $\mathrm{CHi}-\mathrm{C}$ interactions

\begin{tabular}{|l|l|}
\hline Region & BAC \\
\hline $3 p 24.3$ & RP11-66J2 \\
& RP11-1044H7 \\
\hline $4 q 24$ & RP11-10L12 \\
RP11-671L17 \\
\hline $11 q 14.1$ & RP11-1149C10 \\
& RP11-767F3 \\
\hline $15 q 22.3$ & RP11-962J19 \\
\hline $15 q 25.2$ & RP11-106C19 \\
\hline $16 q 12.1$ & RP11-418F16 \\
\hline $16 q 23.1$ & RP11-625L17 \\
\hline & RP11-1113K6 \\
\hline
\end{tabular}


Supplementary Table 7. PCR primers used to amplify promoter-control interactions in $3 \mathrm{C}$ validation of selected $\mathrm{CHi}-\mathrm{C}$ interactions.

\begin{tabular}{|l|l|}
\hline Primer & Sequence $\left(\mathbf{5}^{\prime}\right.$-3') \\
\hline $3 p 24.3$ promoter & ACCTACCCCATCACTCTTACTCCCTTTATC \\
\hline $3 p 24.3$ control & AAGATGGGAATTTGTAAATGCAGCAGTGT \\
\hline $4 q 24$ promoter & TACAGACTCAGATGAAGTTCCATGCCACAG \\
\hline $4 q 24$ control & CTGTTGCTCCGTACCCTTGCCAAGATTTAG \\
\hline $11 q 14.1$ promoter & CCTGTCTGGGAGTTGAGGGTTTGTGGCC \\
\hline $11 q 14.1$ control & GGGGTCTGGGAGCTTCACCTGAAAAGTAAC \\
\hline $15 q 22.3$ promoter & TGTTCTCTTCACTCATGCACTCTAGCCACA \\
\hline $15 q 22.3$ control & TACTTGTGAAAGAGATGACTGTGTGGCCCT \\
\hline $15 q 25.2$ promoter & CCAAGTTGTGTTTATGTATCTCAGGAGG \\
\hline $15 q 25.2$ control & ATGTTGTGTATCCTTTCATAGCAATTCT \\
\hline $16 q 12.1$ promoter & TCAGTATGGTTATTTCACTTTCCATAGACA \\
\hline $16 q 12.1$ control & CGTGGTTCTAATAGGAAGTTCTTGGTT \\
\hline $16 q 23.1$ promoter & AATAAATTGTTAGTTGTAGAATTAGGTGG \\
\hline $16 q 23.1$ control & GTATAAAAGAAGTCATCATGGTACTCAAG \\
\hline
\end{tabular}


Supplementary Table 8. PCR primers used to amplify promoter-element interactions in $3 \mathrm{C}$ validation of selected $\mathrm{CHi}-\mathrm{C}$ interactions.

\begin{tabular}{|c|c|}
\hline Primer & Sequence $\left(5^{\prime}-3^{\prime}\right)$ \\
\hline $3 p 24.3$ promoter & ATCTCAGCCAAGGTGTCATCACTGGAGAG \\
\hline $3 p 24.3$ element & TGGAGACATAGCCCAAGGCTCTTAAACTCA \\
\hline $4 q 24$ promoter & TACAGACTCAGATGAAGTTCCATGCCACAG \\
\hline 4q24 element & AGCTCCACTGTACTCCACACCTACTTCCT \\
\hline 11q14.1 promoter & GGTTCTAAAGGGTGCACTGTGGCTTTGA \\
\hline 11q14.1 element & TGCATTTGGAGCTGTCCCTTAATACTGGA \\
\hline $15 q 22.3$ promoter & TGTTCTCTTCACTCATGCACTCTAGCCACA \\
\hline $15 q 22.3$ element & AGCTGGTAGGAAGGTGGTTAATGGAGAGTT \\
\hline $15 q 25.2$ promoter & TCCCTAAACCACACCCACTCCCATTGTACC \\
\hline $15 q 25.2$ element & AGTAGGGGCTTTATGAATGGTTGTGCATCC \\
\hline 16q12.1 promoter & GGAATATCAGTATGGTTATTTCACTTTCCA \\
\hline 16q12.1 element & CACATGTACTAAGGGTTGAGATCCAAGA \\
\hline $16 q 23.1$ promoter & CAATTGTACTGACTTTTCTGTGTATCTGGA \\
\hline 16q23.1 element & CTTCATGAGCCATCACTAGAGAAACAGTA \\
\hline
\end{tabular}


Supplementary Table 9 - All possible eQTL associations per locus.

Listed in bold font are the eQTL associations reported in this manuscript, which were supported by either promoter variants or looping Hi-C contacts from the eQTL SNP (putative enhancer) to the eQTL gene promoter. For reference purposes all other possible variant/gene eQTL results at these loci are also listed below (non-bold), ignoring the $\mathrm{Hi}-\mathrm{C}$ contact/promoter variant data, and using the following criteria: i) genes within $1 \mathrm{Mb}$ \& variants within $\mathrm{R}^{2}>0.8$ of sentinal SNP, ii) $P<0.05$, iii) same RNA-seq dataset.

\begin{tabular}{|c|c|c|c|c|c|c|c|}
\hline Locus (Cytoband) & $\begin{array}{l}\text { Reported sentinal SNP } \\
\text { (strongest association } \\
\text { with TGCT) }\end{array}$ & $\begin{array}{l}P \text {-value } \\
\text { association with } \\
\text { TGCT }\end{array}$ & eQTL gene & eQTL SNP & $\begin{array}{l}P \text {-value for } \\
\text { eQTL } \\
\text { association }\end{array}$ & \begin{tabular}{|l|}
$P$-value \\
association with \\
TGCT
\end{tabular} & $\begin{array}{l}\text { RNA-seq } \\
\text { dataset }\end{array}$ \\
\hline $14 q 22.3$ & rs1009647 & $3.4 \mathrm{E}-08$ & ATG14 & rs1538257 & $1.7 \mathrm{E}-05$ & 1.7E-05 & GTEX \\
\hline $14 q 22.3$ & rs1009647 & $3.4 \mathrm{E}-08$ & $R P 11-665 C 16.6$ & rs34727214 & $6.0 \mathrm{E}-08$ & $2.1 \mathrm{E}-06$ & GTEX \\
\hline $14 q 22.3$ & rs1009647 & $3.4 \mathrm{E}-08$ & $R P 11-665 C 16.6$ & rs12885227 & $6.3 \mathrm{E}-08$ & $2.9 \mathrm{E}-07$ & GTEX \\
\hline $14 q 22.3$ & rs1009647 & $3.4 \mathrm{E}-08$ & $R P 11-665 C 16.6$ & rs12885245 & $6.3 \mathrm{E}-08$ & $3.0 \mathrm{E}-07$ & GTEx \\
\hline $14 q 22.3$ & rs1009647 & $3.4 \mathrm{E}-08$ & RP11-665C16.6 & rs7153619 & $9.7 \mathrm{E}-08$ & $2.2 \mathrm{E}-06$ & GTEX \\
\hline $14 q 22.3$ & rs1009647 & $3.4 \mathrm{E}-08$ & RP11-665C16.6 & rs35502084 & $1.6 \mathrm{E}-07$ & $3.5 \mathrm{E}-07$ & GTEX \\
\hline $14 q 22.3$ & rs1009647 & $3.4 \mathrm{E}-08$ & RP11-665C16.6 & rs1009647 & $5.8 \mathrm{E}-07$ & 5.0E-07 & GTEX \\
\hline $14 q 22.3$ & rs1009647 & $3.4 \mathrm{E}-08$ & RP11-665C16.6 & rs1009648 & $8.6 \mathrm{E}-07$ & $3.2 \mathrm{E}-06$ & GTEX \\
\hline $14 q 22.3$ & rs1009647 & $3.4 \mathrm{E}-08$ & RP11-665C16.6 & rs946056 & $8.6 \mathrm{E}-07$ & $3.2 \mathrm{E}-06$ & GTEX \\
\hline $14 q 22.3$ & rs1009647 & $3.4 \mathrm{E}-08$ & RP11-665C16.6 & rs1890256 & $8.7 \mathrm{E}-07$ & $4.6 \mathrm{E}-07$ & GTEx \\
\hline $16 q 12.1$ & rs8046148 & 4.5E-07 & HEATR3 & rs12930079 & $6.0 \mathrm{E}-26$ & 1.0E-09 & GTEx \\
\hline $16 q 12.1$ & rs8046148 & 4.5E-07 & HEATR3 & rs1008815 & $5.5 \mathrm{E}-12$ & 1.7E-06 & GTEX \\
\hline $16 q 12.1$ & rs8046148 & 4.5E-07 & HEATR3 & rs2356837 & $5.5 E-12$ & 3.2E-06 & GTEX \\
\hline $16 q 12.1$ & rs8046148 & 4.5E-07 & HEATR3 & rs8047421 & $5.2 E-12$ & 2.9E-06 & GTEX \\
\hline $16 q 12.1$ & rs8046148 & 4.5E-07 & HEATR3 & rs2058813 & $5.5 E-12$ & 2.9E-06 & GTEX \\
\hline $16 q 12.1$ & rs8046148 & 4.5E-07 & HEATR3 & rs8062151 & $5.2 \mathrm{E}-12$ & 3.0E-06 & GTEX \\
\hline $16 q 12.1$ & rs8046148 & 4.5E-07 & HEATR3 & rs11076512 & $2.7 \mathrm{E}-12$ & 3.0E-06 & GTEX \\
\hline $16 q 12.1$ & rs8046148 & 4.5E-07 & HEATR3 & rs11642579 & $4.6 \mathrm{E}-12$ & 3.0E-06 & GTEX \\
\hline $16 q 12.1$ & rs8046148 & $4.5 \mathrm{E}-07$ & HEATR3 & rs4785381 & $4.0 \mathrm{E}-12$ & 3.1E-06 & GTEX \\
\hline $16 q 12.1$ & rs8046148 & $4.5 \mathrm{E}-07$ & HEATR3 & rs11355227 & $4.0 \mathrm{E}-12$ & 3.1E-06 & GTEX \\
\hline $16 q 12.1$ & rs8046148 & $4.5 \mathrm{E}-07$ & HEATR3 & rs11640627 & 3.3E-12 & $3.5 \mathrm{E}-06$ & GTEX \\
\hline $16 q 12.1$ & rs8046148 & $4.5 \mathrm{E}-07$ & HEATR3 & rs8052350 & 2.6E-12 & $3.8 \mathrm{E}-06$ & GTEX \\
\hline $16 q 12.1$ & rs8046148 & $4.5 \mathrm{E}-07$ & HEATR3 & rs1558813 & 3.2E-12 & $3.5 \mathrm{E}-06$ & GTEX \\
\hline $16 q 12.1$ & rs8046148 & 4.5E-07 & HEATR3 & rs12934889 & 4.4E-12 & 3.7E-06 & GTEX \\
\hline $16 q 12.1$ & rs8046148 & $4.5 \mathrm{E}-07$ & HEATR3 & rs4785382 & 7.5E-14 & $3.5 \mathrm{E}-07$ & GTEX \\
\hline $16 q 12.1$ & rs8046148 & $4.5 \mathrm{E}-07$ & HEATR3 & rs8045354 & $2.8 \mathrm{E}-12$ & 9.7E-07 & GTEX \\
\hline $16 q 12.1$ & rs8046148 & $4.5 \mathrm{E}-07$ & HEATR3 & rs8046148 & 4.1E-12 & 5.2E-07 & GTEX \\
\hline $16 q 12.1$ & rs8046148 & 4.5E-07 & HEATR3 & rs9933767 & 4.0E-12 & 5.7E-07 & GTEX \\
\hline $16 q 12.1$ & rs8046148 & $4.5 \mathrm{E}-07$ & HEATR3 & rs4632126 & 2.6E-11 & 5.7E-07 & GTEX \\
\hline $16 q 23.1$ & rs4888262 & $6.9 \mathrm{E}-12$ & RFWD3 & rs58136167 & $7.6 \mathrm{E}-24$ & 2.0E-06 & GTEx \\
\hline $16 q 23.1$ & rs4888262 & $6.9 \mathrm{E}-12$ & RFWD3 & rs11642283 & $1.5 \mathrm{E}-12$ & $1.6 \mathrm{E}-10$ & GTEx \\
\hline $16 q 23.1$ & rs4888262 & $6.9 \mathrm{E}-12$ & RFWD3 & rs12716769 & $1.4 \mathrm{E}-10$ & 9.5E-13 & GTEx \\
\hline $16 q 23.1$ & rs4888262 & $6.9 \mathrm{E}-12$ & RFWD3 & rs150095922 & $1.9 \mathrm{E}-12$ & $8.3 \mathrm{E}-13$ & GTEx \\
\hline $16 q 23.1$ & rs4888262 & $6.9 \mathrm{E}-12$ & RFWD3 & rs8058133 & 4.0E-14 & 8.3E-13 & GTEX \\
\hline $16 q 23.1$ & rs4888262 & $6.9 \mathrm{E}-12$ & RFWD3 & rs8052367 & 4.6E-14 & $5.0 \mathrm{E}-13$ & GTEx \\
\hline $16 q 23.1$ & rs4888262 & $6.9 \mathrm{E}-12$ & RFWD3 & rs7188880 & $2.1 \mathrm{E}-12$ & $1.2 \mathrm{E}-11$ & GTEx \\
\hline $16 q 23.1$ & rs4888262 & $6.9 \mathrm{E}-12$ & RFWD3 & rs9929496 & $1.5 \mathrm{E}-12$ & $1.3 \mathrm{E}-11$ & GTEx \\
\hline $16 q 23.1$ & rs4888262 & $6.9 \mathrm{E}-12$ & RFWD3 & rs9930188 & 2.7E-11 & $1.5 \mathrm{E}-11$ & GTEx \\
\hline $16 q 23.1$ & rs4888262 & $6.9 \mathrm{E}-12$ & RFWD3 & rs9929931 & $1.5 \mathrm{E}-11$ & 2.0E-11 & GTEX \\
\hline $16 q 23.1$ & rs4888262 & $6.9 \mathrm{E}-12$ & RFWD3 & rs9922988 & $1.2 \mathrm{E}-11$ & $4.5 \mathrm{E}-11$ & GTEX \\
\hline $16 q 23.1$ & rs4888262 & $6.9 \mathrm{E}-12$ & RFWD3 & rs4888262 & $1.5 \mathrm{E}-12$ & $1.2 \mathrm{E}-11$ & GTEX \\
\hline $16 q 23.1$ & rs4888262 & $6.9 \mathrm{E}-12$ & RFWD3 & rs7188581 & 3.2E-11 & 6.9E-12 & GTEX \\
\hline
\end{tabular}




\begin{tabular}{|c|c|c|c|c|c|c|c|}
\hline Locus (Cytoband) & $\begin{array}{l}\text { Reported sentinal SNP } \\
\text { (strongest association } \\
\text { with TGCT) }\end{array}$ & $\begin{array}{l}P \text {-value } \\
\text { association with } \\
\text { TGCT }\end{array}$ & eQTL gene & eQTL SNP & $\begin{array}{l}P \text {-value for } \\
\text { eQTL } \\
\text { association }\end{array}$ & \begin{tabular}{|l|}
$P$-value \\
association with \\
TGCT
\end{tabular} & $\begin{array}{l}\text { RNA-seq } \\
\text { dataset }\end{array}$ \\
\hline $16 q 23.1$ & rs4888262 & $6.9 \mathrm{E}-12$ & RFWD3 & rs8059780 & $3.6 \mathrm{E}-12$ & $1.6 \mathrm{E}-11$ & GTEX \\
\hline $16 q 23.1$ & rs4888262 & $6.9 \mathrm{E}-12$ & RFWD3 & rs12924948 & $2.5 \mathrm{E}-11$ & $1.1 \mathrm{E}-11$ & GTEx \\
\hline $16 q 23.1$ & rs4888262 & $6.9 \mathrm{E}-12$ & RFWD3 & rs4888264 & $9.4 \mathrm{E}-13$ & $1.3 \mathrm{E}-11$ & GTEx \\
\hline $16 q 23.1$ & rs4888262 & $6.9 \mathrm{E}-12$ & RFWD3 & rs28681530 & $1.5 \mathrm{E}-12$ & $1.0 \mathrm{E}-11$ & GTEX \\
\hline $16 q 23.1$ & rs4888262 & $6.9 \mathrm{E}-12$ & RFWD3 & rs4888265 & $1.6 \mathrm{E}-12$ & $8.5 \mathrm{E}-12$ & GTEX \\
\hline $16 q 23.1$ & rs4888262 & $6.9 \mathrm{E}-12$ & RFWD3 & rs9923145 & $1.5 \mathrm{E}-12$ & $1.0 \mathrm{E}-11$ & GTEx \\
\hline $16 q 23.1$ & rs4888262 & $6.9 \mathrm{E}-12$ & RFWD3 & rs4888267 & $1.5 \mathrm{E}-12$ & $1.0 \mathrm{E}-11$ & GTEx \\
\hline $16 q 23.1$ & rs4888262 & $6.9 \mathrm{E}-12$ & RFWD3 & rs7191665 & $1.5 \mathrm{E}-12$ & $1.1 \mathrm{E}-11$ & GTEX \\
\hline $16 q 23.1$ & rs4888262 & $6.9 \mathrm{E}-12$ & RFWD3 & rs8062783 & $1.5 \mathrm{E}-12$ & $1.0 \mathrm{E}-11$ & GTEX \\
\hline $16 q 23.1$ & rs4888262 & $6.9 \mathrm{E}-12$ & RFWD3 & rs8061942 & $1.5 \mathrm{E}-12$ & $1.1 \mathrm{E}-11$ & GTEX \\
\hline $16 q 23.1$ & rs4888262 & $6.9 \mathrm{E}-12$ & RFWD3 & rs7201320 & $1.5 \mathrm{E}-12$ & $9.8 \mathrm{E}-12$ & GTEX \\
\hline $16 q 23.1$ & rs4888262 & $6.9 \mathrm{E}-12$ & RFWD3 & rs56099065 & 9.9E-11 & 9.1E-10 & GTEx \\
\hline $16 q 23.1$ & rs4888262 & $6.9 \mathrm{E}-12$ & RFWD3 & rs9931225 & $1.4 \mathrm{E}-11$ & $3.0 \mathrm{E}-10$ & GTEX \\
\hline $16 q 23.1$ & rs4888262 & $6.9 \mathrm{E}-12$ & RFWD3 & rs62053585 & $1.3 \mathrm{E}-06$ & $2.3 \mathrm{E}-07$ & GTEX \\
\hline $16 q 23.1$ & rs4888262 & $6.9 \mathrm{E}-12$ & RFWD3 & rs4888271 & $1.7 \mathrm{E}-10$ & $5.9 \mathrm{E}-10$ & GTEX \\
\hline $16 q 23.1$ & rs4888262 & $6.9 \mathrm{E}-12$ & RFWD3 & rs4887783 & $2.7 \mathrm{E}-13$ & $2.9 \mathrm{E}-10$ & GTEX \\
\hline $16 q 23.1$ & rs4888262 & $6.9 \mathrm{E}-12$ & RFWD3 & rs5817922 & $3.0 \mathrm{E}-10$ & $4.6 \mathrm{E}-13$ & GTEX \\
\hline $16 q 23.1$ & rs4888262 & $6.9 \mathrm{E}-12$ & RFWD3 & rs4888274 & $3.3 \mathrm{E}-12$ & 1.3E-12 & GTEX \\
\hline $16 q 23.1$ & rs4888262 & $6.9 \mathrm{E}-12$ & RFWD3 & rs4072222 & $3.3 \mathrm{E}-12$ & $1.6 \mathrm{E}-10$ & GTEx \\
\hline $1 q 22$ & rs2072499 & 1.9E-10 & CCT3 & rs1052067 & $1.2 \mathrm{E}-06$ & $3.3 \mathrm{E}-08$ & GTEx \\
\hline $1 q 22$ & rs2072499 & $1.9 \mathrm{E}-10$ & $N / A$ & $\mathrm{~N} / \mathrm{A}$ & N/A & & GTEx \\
\hline $17 q 22$ & rs9905704 & $3.4 \mathrm{E}-20$ & TEX14 & rs654778 & $4.9 \mathrm{E}-07$ & $3.1 E-20$ & GTEx \\
\hline $17 q 22$ & rs9905704 & $3.4 \mathrm{E}-20$ & $N / A$ & $\mathrm{~N} / \mathrm{A}$ & N/A & & GTEx \\
\hline $4 q 22.3$ & rs17021463 & 3.3E-08 & SMARCAD1 & rs2865350 & $6.4 \mathrm{E}-03$ & 1.8E-07 & TCGA \\
\hline $4 q 22.3$ & rs17021463 & 3.3E-08 & ATOH1 & rs2865350 & $3.7 \mathrm{E}-02$ & & TCGA \\
\hline $4 q 24$ & rs2720460 & $6.6 \mathrm{E}-20$ & MANBA & rs2720460 & $1.7 \mathrm{E}-02$ & 4.8E-20 & TCGA \\
\hline $4 q 24$ & rs2720460 & $6.6 \mathrm{E}-20$ & $N / A$ & $\mathrm{~N} / \mathrm{A}$ & N/A & & TCGA \\
\hline $6 p 21.31$ & rs210138 & 3.5E-37 & BAK1 & rs210138 & $2.0 \mathrm{E}-02$ & 2.9E-37 & TCGA \\
\hline $6 p 21.31$ & rs210138 & $3.5 \mathrm{E}-37$ & ITPR3 & rs210138 & 3.1E-02 & $2.9 \mathrm{E}-37$ & TCGA \\
\hline $6 p 21.31$ & rs210138 & $3.5 \mathrm{E}-37$ & $H L A-D O B$ & rs210138 & $1.3 \mathrm{E}-03$ & $2.9 \mathrm{E}-37$ & TCGA \\
\hline $6 p 21.31$ & rs210138 & $3.5 \mathrm{E}-37$ & $H L A-D O B$ & rs210138 & 9.5E-04 & $2.9 \mathrm{E}-37$ & TCGA \\
\hline $8 p 23.1$ & rs17153755 & 4.4E-08 & GATA4 & rs1004712 & 3.2E-02 & 1.7E-09 & TCGA \\
\hline $8 p 23.1$ & rs17153755 & 4.4E-08 & GATA4 & rs1466785 & 4.3E-02 & $3.5 \mathrm{E}-06$ & TCGA \\
\hline $8 p 23.1$ & rs17153755 & 4.4E-08 & FDFT1 & rs1004712 & $5.2 \mathrm{E}-03$ & 1.7E-09 & TCGA \\
\hline $8 p 23.1$ & rs17153755 & 4.4E-08 & FDFT1 & rs1466785 & 7.7E-03 & $3.5 \mathrm{E}-06$ & TCGA \\
\hline $8 p 23.1$ & rs17153755 & 4.4E-08 & ZNF705D & rs1466785 & $1.0 \mathrm{E}-02$ & $3.5 \mathrm{E}-06$ & TCGA \\
\hline $8 p 23.1$ & rs17153755 & 4.4E-08 & ZNF705D & rs1004712 & $1.3 \mathrm{E}-02$ & 1.7E-09 & TCGA \\
\hline $8 p 23.1$ & rs17153755 & 4.4E-08 & CTSB & rs1466785 & 2.2E-02 & $3.5 \mathrm{E}-06$ & TCGA \\
\hline $8 p 23.1$ & rs17153755 & 4.4E-08 & SOX7 & rs17153755 & $2.8 \mathrm{E}-02$ & $1.5 \mathrm{E}-08$ & TCGA \\
\hline $8 p 23.1$ & rs17153755 & 4.4E-08 & DEFB135 & rs1466785 & $3.6 \mathrm{E}-02$ & $3.5 \mathrm{E}-06$ & TCGA \\
\hline $8 p 23.1$ & rs17153755 & 4.4E-08 & LONRF1 & rs1004712 & $3.9 \mathrm{E}-02$ & 1.7E-09 & TCGA \\
\hline $15 q 22.31$ & rs11071896 & 8.4E-13 & SNAPC5 & rs11629783 & $3.2 \mathrm{E}-02$ & $5.3 E-10$ & TCGA \\
\hline
\end{tabular}




\begin{tabular}{|c|c|c|c|c|c|c|c|}
\hline Locus (Cytoband) & $\begin{array}{l}\text { Reported sentinal SNP } \\
\text { (strongest association } \\
\text { with TGCT) }\end{array}$ & $\begin{array}{l}P \text {-value } \\
\text { association with } \\
\text { TGCT }\end{array}$ & eQTL gene & eQTL SNP & $\begin{array}{l}P \text {-value for } \\
\text { eQTL } \\
\text { association }\end{array}$ & \begin{tabular}{|l|}
$P$-value \\
association with \\
TGCT
\end{tabular} & $\begin{array}{l}\text { RNA-seq } \\
\text { dataset }\end{array}$ \\
\hline $15 q 22.31$ & rs11071896 & $8.4 \mathrm{E}-13$ & $N / A$ & N/A & $N / A$ & & TCGA \\
\hline $15 q 25.2$ & rs56046484 & 4.6E-08 & WDR73 & rs2304416 & $3.2 \mathrm{E}-02$ & 1.0E-06 & TCGA \\
\hline $15 q 25.2$ & rs56046484 & 4.6E-08 & SH3GL3 & rs2304416 & $4.8 \mathrm{E}-02$ & 1.0E-06 & TCGA \\
\hline $15 q 25.2$ & rs56046484 & $4.6 \mathrm{E}-08$ & SH3GL3 & rs17541572 & 4.1E-02 & 5.9E-05 & TCGA \\
\hline
\end{tabular}


Supplementary Table 10. Transcription factor motif enrichment.

\begin{tabular}{|l|rc|}
\hline TF Motif & $\begin{array}{c}\text { Fold- } \\
\text { Enrichment }\end{array}$ & $P$-value \\
\hline GATA & 1.8 & $1.2 \mathrm{E}-02$ \\
KLF4 & 1.8 & $1.2 \mathrm{E}-03$ \\
NANOG & 1.8 & $5.0 \mathrm{E}-02$ \\
LHX8 & 3.0 & $3.2 \mathrm{E}-02$ \\
SOX2 & 2.5 & $2.4 \mathrm{E}-03$ \\
POU5F1 & 1.8 & $6.0 \mathrm{E}-03$ \\
DMRT1 & 1.8 & $4.1 \mathrm{E}-02$ \\
SOX9 & 1.3 & $2.5 \mathrm{E}-01$ \\
PRDM1 & 1.3 & $2.2 \mathrm{E}-01$ \\
CTCF & 1.8 & $2.4 \mathrm{E}-03$ \\
\hline
\end{tabular}


Supplementary Figure 1 - Quantile-quantile plot

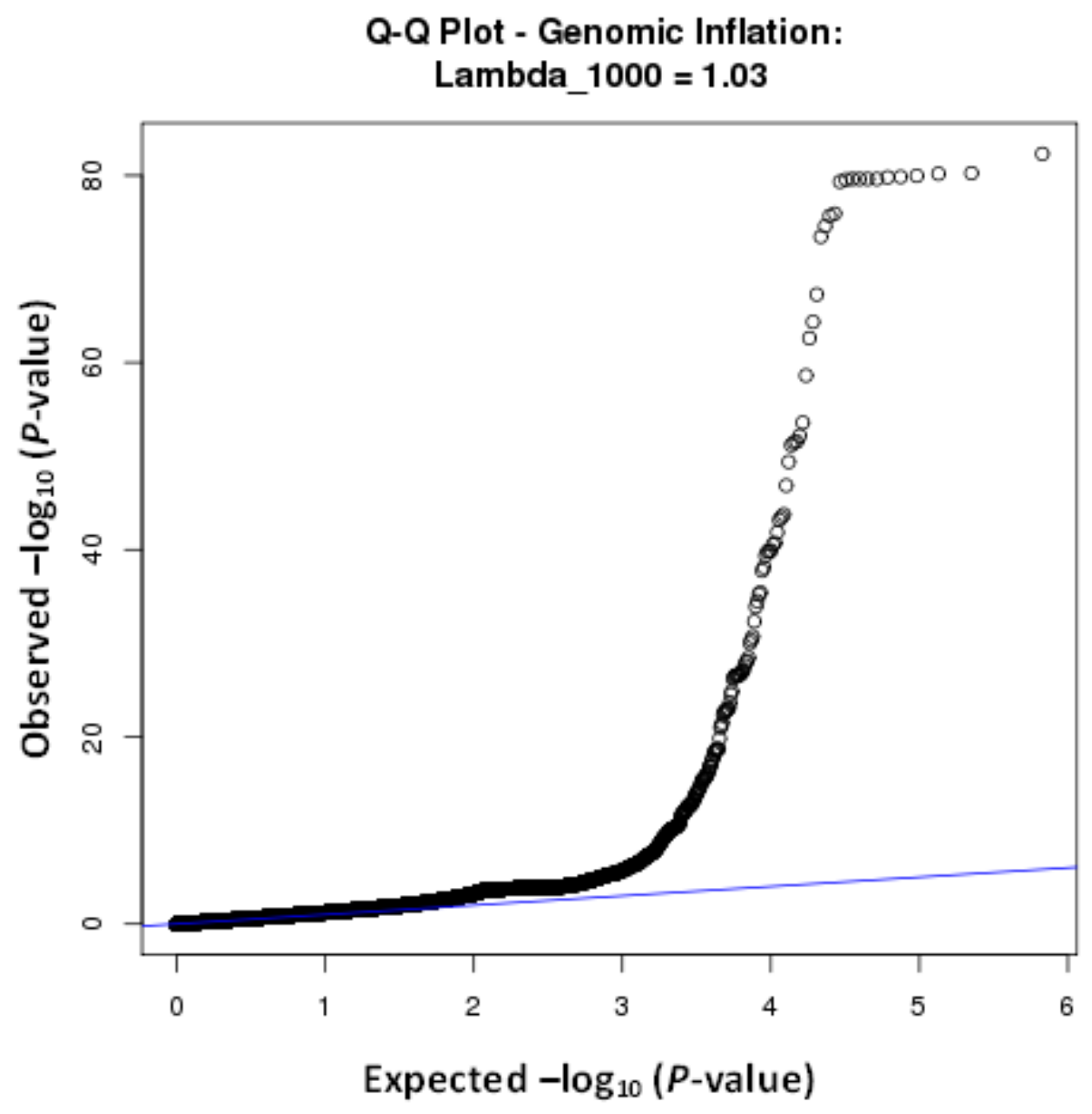




\section{Supplementary Figure 2 - Evidence of tissue specific histone mark enrichment.}

The heatmap shows enrichment scores for histone marks H3k4me3, H3k9ac, H3k4me1 and $\mathrm{H} 3 \mathrm{k} 9 \mathrm{me}$, using ChIP-Seq data from 42 encode cell-types. Enrichment is measured as the fold-increase in ChIP-Seq signal peaks at the TGCT risk loci compared to a series of randomly generated null distributions. The key markers of functionally active chromatin, H3K4me3, H3K9ac and H3K4me1 (first 3 columns), were most strongly enriched in the Nt2d1 TGCT cell line. White coloring means no data was available.

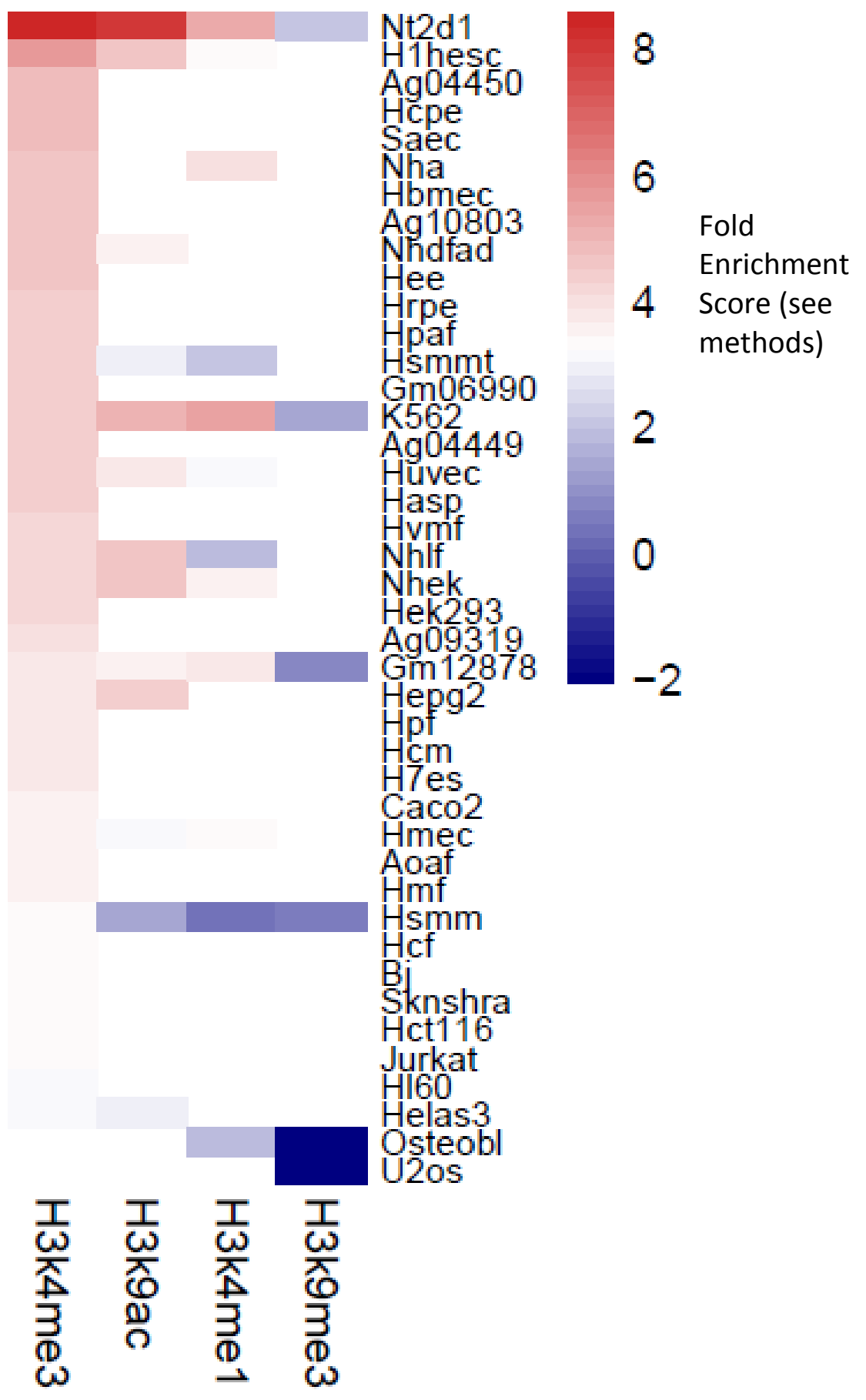


Supplementary Figure 3 - Visualisation of significant eQTL associations. The first five plots are for associations in normal testicular tissue, the remaining six are in TGC tumor tissue. Each plot shows a set of three boxplots of rank-normalised RNA-seq gene expression values for the given gene ( $Y$-axis), split by genotype (X-axis) of the SNP with strongest eQTL association at each risk locus. X-axis indicates the number of individuals for each given genotype.
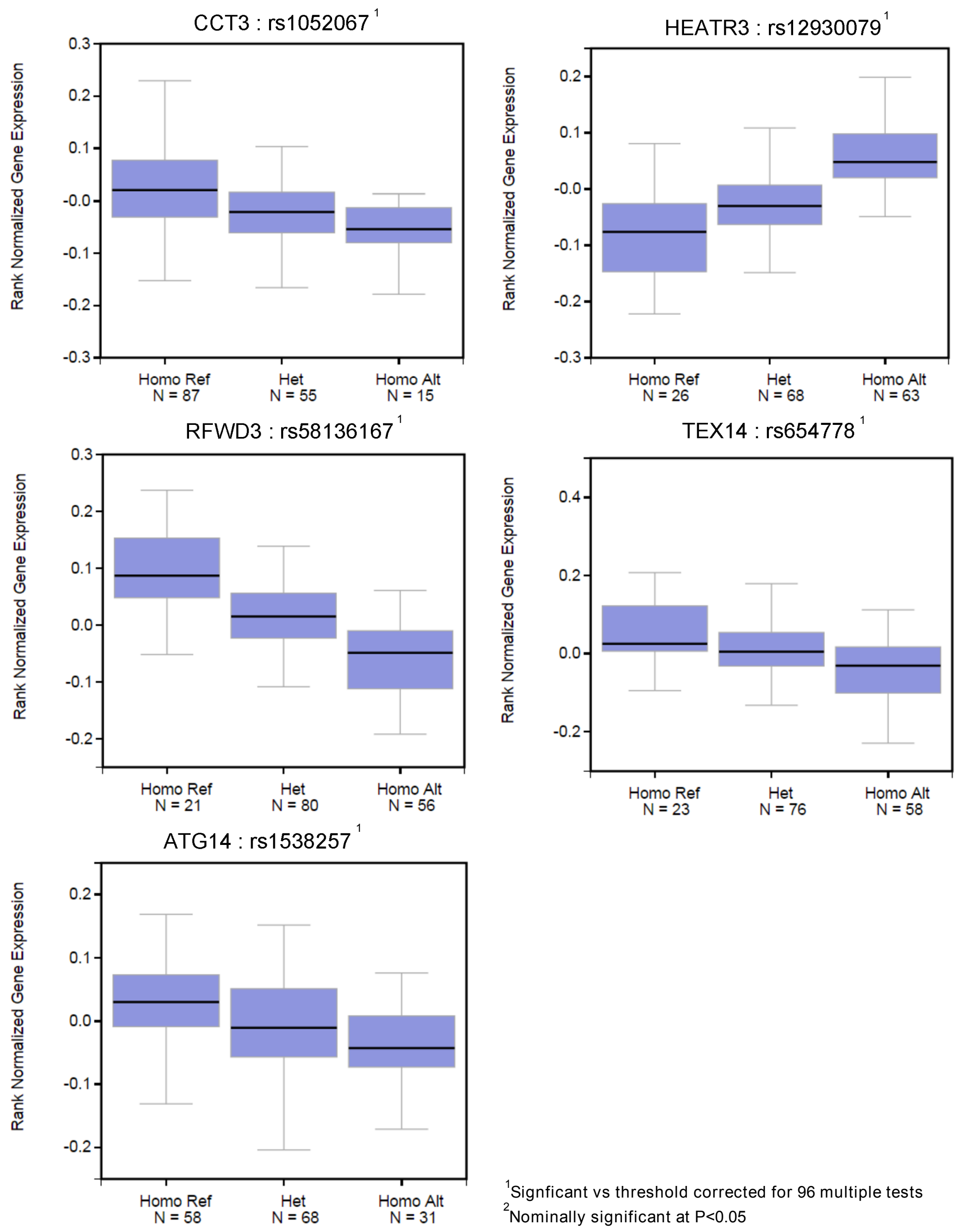

${ }^{1}$ Signficant vs threshold corrected for 96 multiple tests

${ }^{2}$ Nominally significant at $\mathrm{P}<0.05$ 
SMARCAD1 : rs 11629783

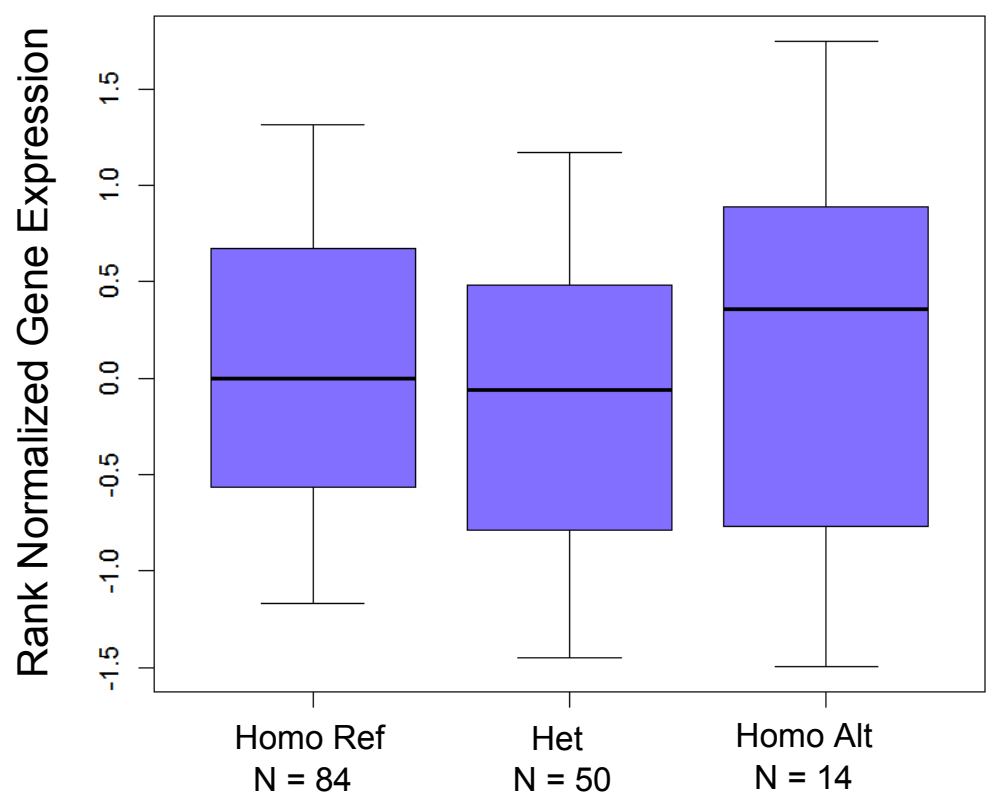

SNAPC5 : rs11629783 ${ }^{2}$

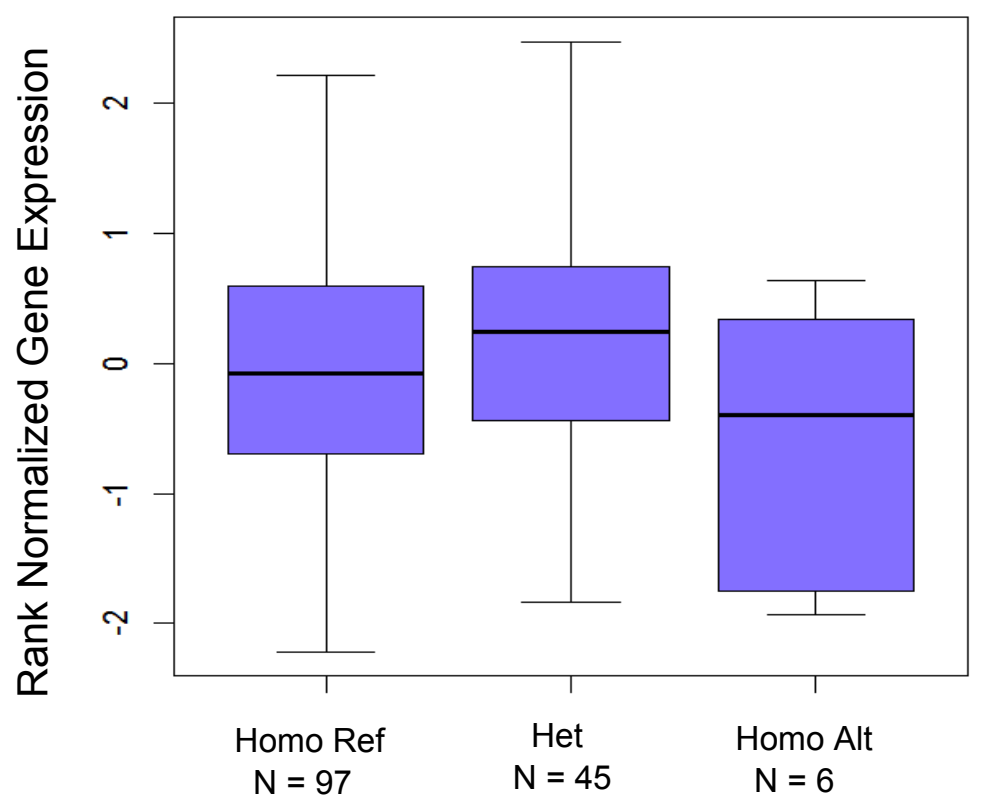

WDR73 : rs2304416 ${ }^{2}$

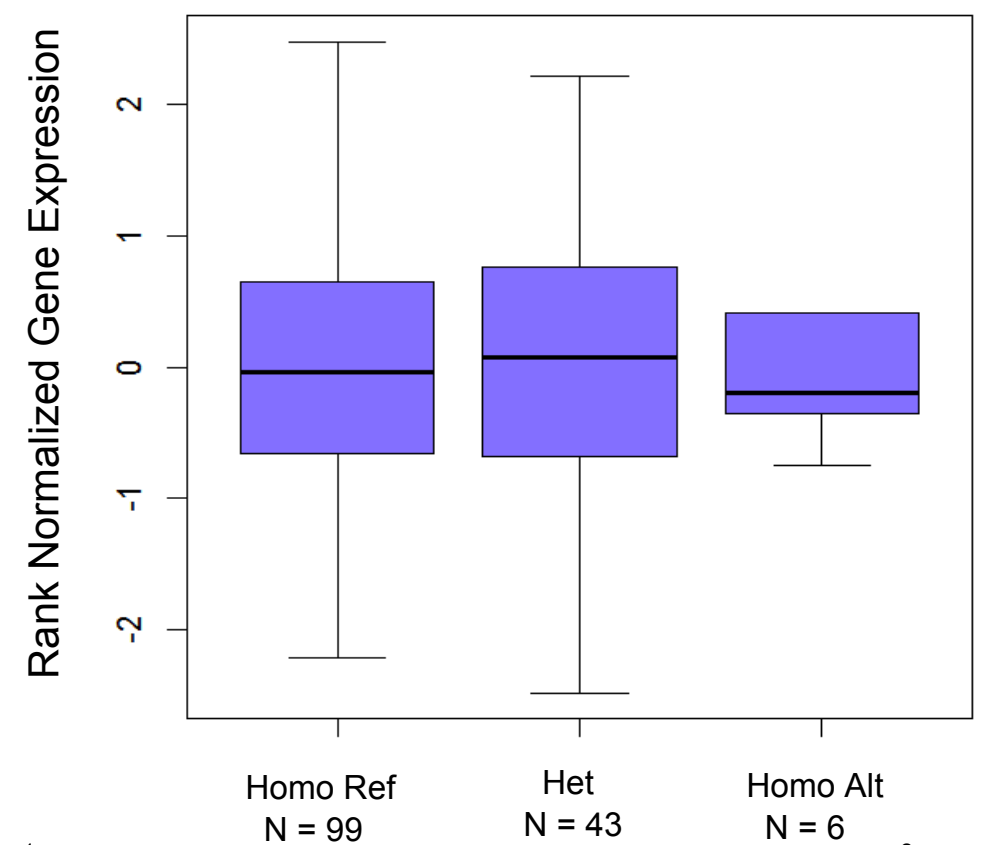

$B A K 1: \mathrm{rs} 210138^{2}$

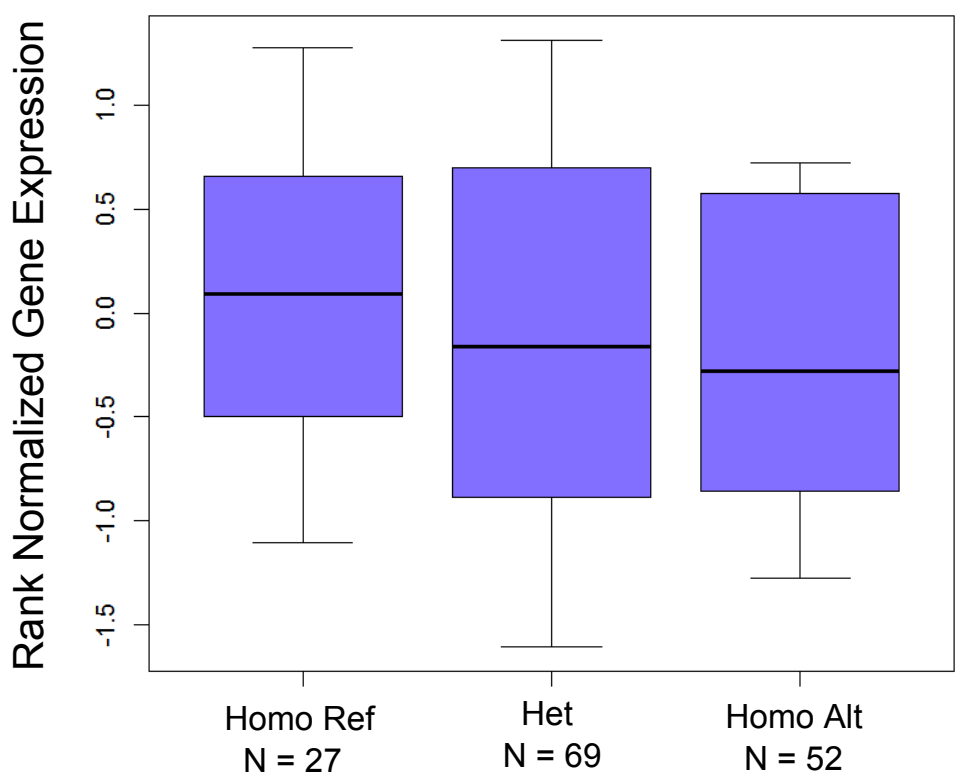

GATA4 : rs $1004712^{2}$

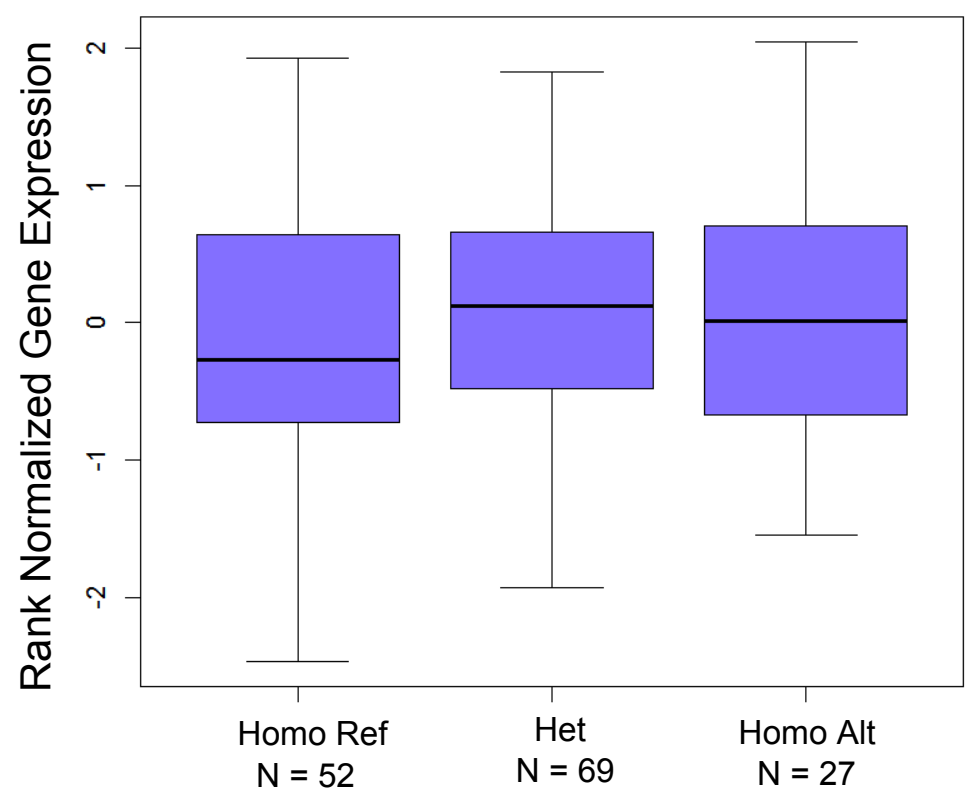

MANBA : rs $2720460^{2}$

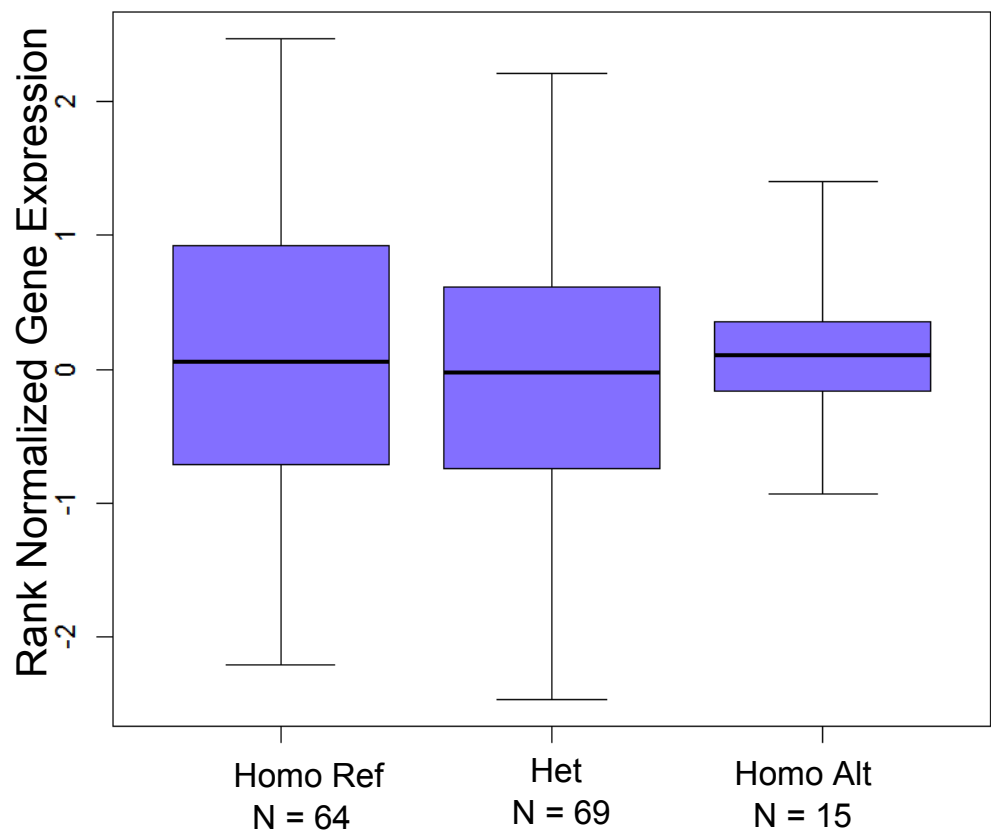

${ }^{1}$ Significant vs threshold corrected for 96 multiple tests $\quad{ }^{2}$ Nominally significant at $\mathrm{P}<0.05$ 


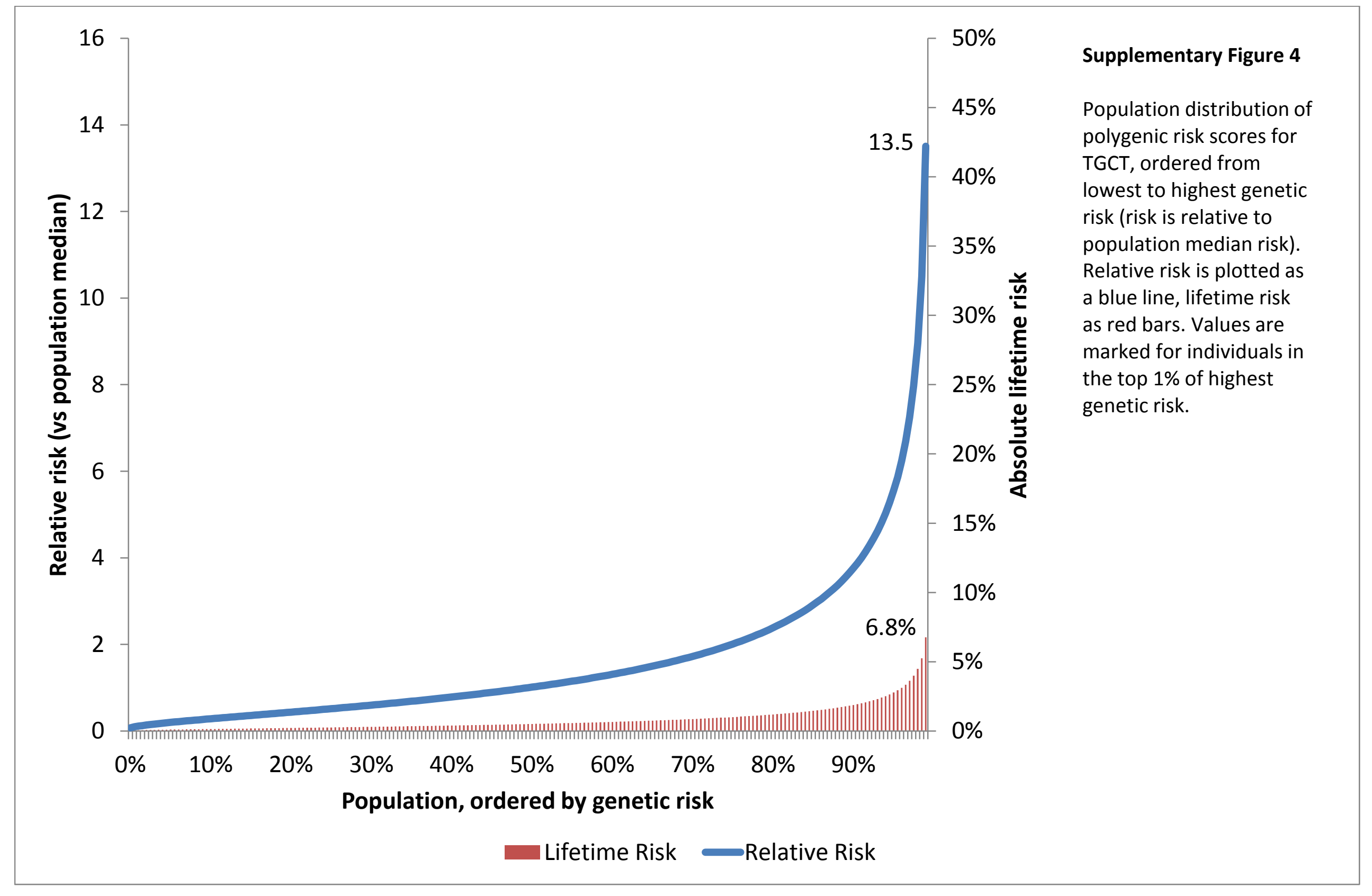


Supplementary Figure 5

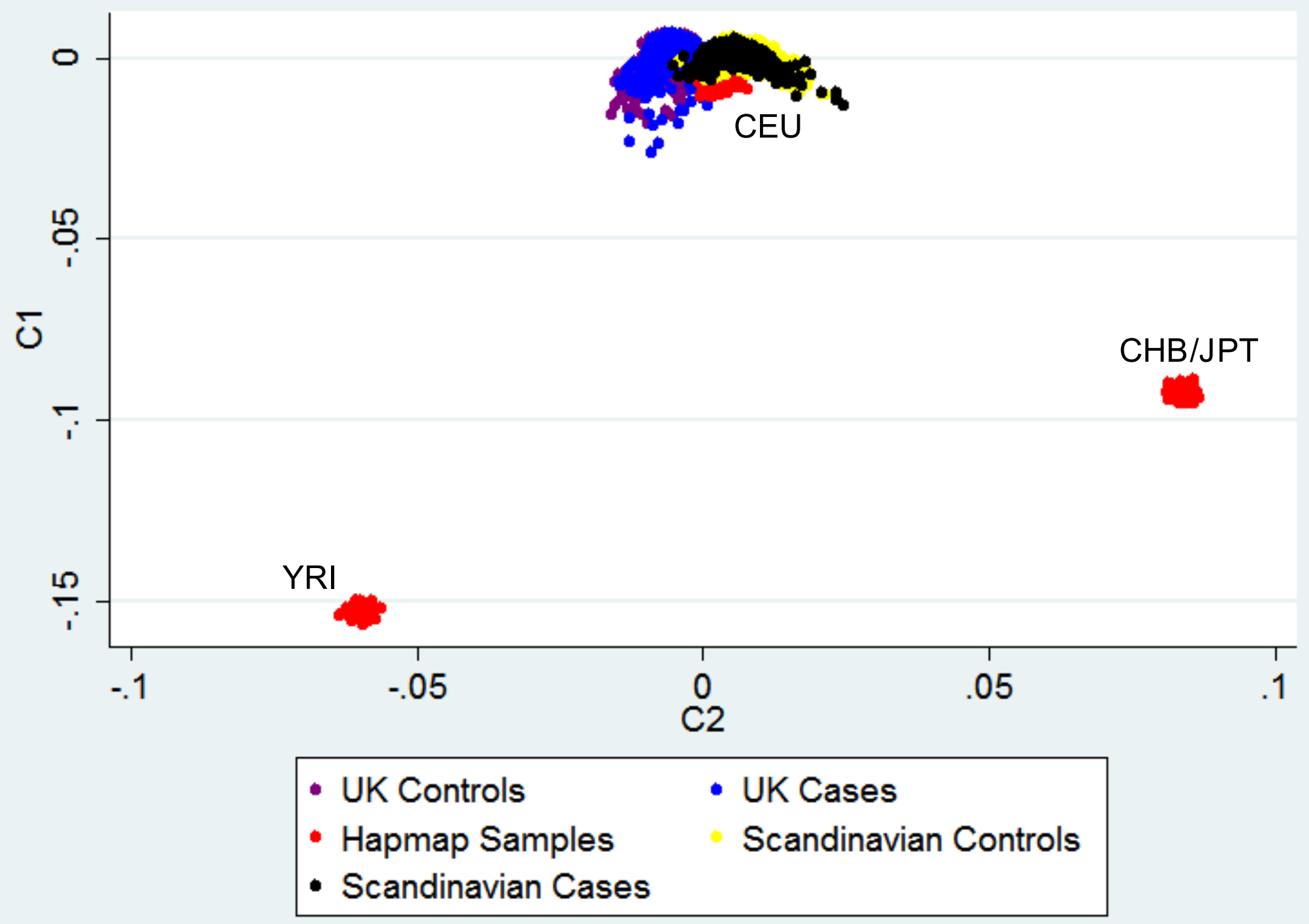


Supplementary Figure 6 - Regional plots of remaining 16 new TGCT loci, not depicted in main text.

The - $\log 10 P$ values of genotyped SNPs based on Oncoarray data (triangles) and imputed SNPS

(circles) are plotted alongside recombination rates (centi-morgans per mega-base). The intensity of red shading indicates the strength of LD with the labelled sentinel SNP. Gene transcripts within the region are shown below. Below the gene transcripts are $\mathrm{Hi}-\mathrm{C}$ next generation sequencing read pair counts (intervals are determined by HindIII cut points, with average $3 \mathrm{~Kb}$ resolution), where gaps represent bait locations, which are plotted. Looping contacts are depicted in regions with significant $\mathrm{Hi}-\mathrm{C}$ interactions, where colour and depth of ribbons represent the score. Significant $\mathrm{Hi}-\mathrm{C}$ interctions are present in four regions (rs7581030, rs6821144, rs9966612, and rs12481572) and absent in 12 regions (rs648090, rs4931000, rs7315956, rs1009647, rs7404843, rs2241024, rs4599029, rs4240895, rs739525, rs4862848, rs11155671, rs17689040). A zoomed-in section displays the gene transcripts, predicted chromHMM states (coloured as per the legend), and contacts in regions with significant $\mathrm{Hi}-\mathrm{C}$ contacts. 


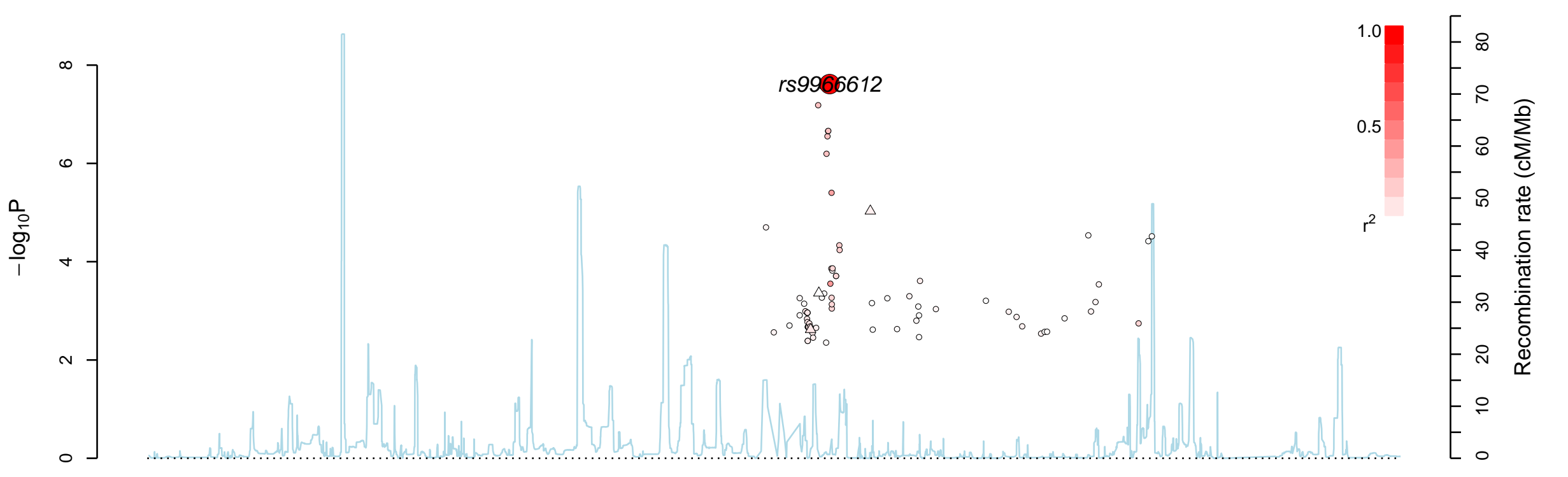

«HFH THOC1

COLEC12 k\#+ +4

\section{CETN1| ENOSF1 배HAH-K}

CLUL1 HHAHA H H

TYMS \#\#

C18orf56 |

YES1 H HAllthereest

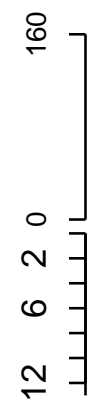

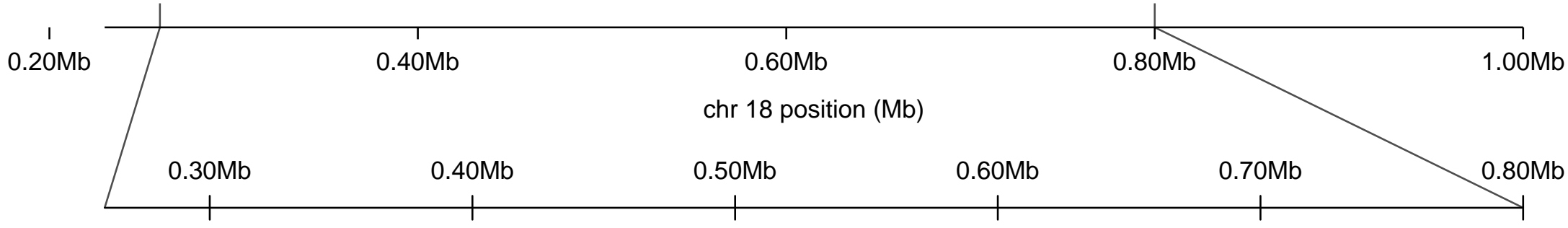

HWI THOC1

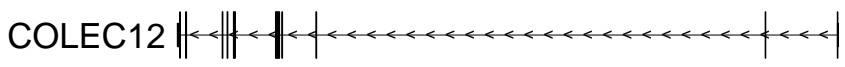

CETN1 |

CLUL11 +中+tHAt+H

C18orf56

ENOSF1 1 HtH HWH + - H
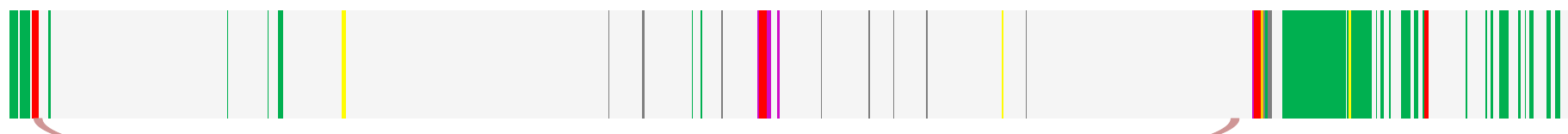

$\sim \exists$

0 - 


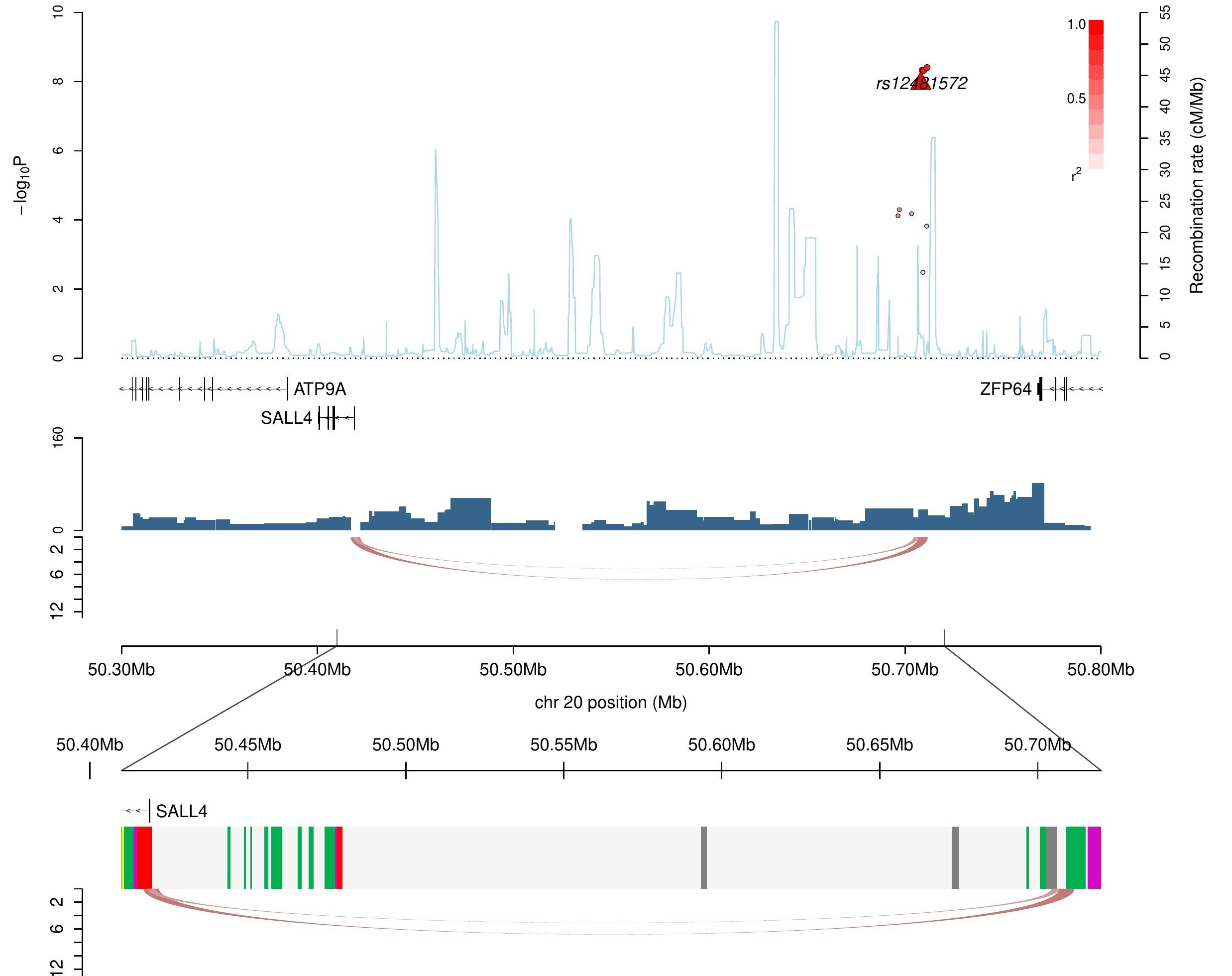




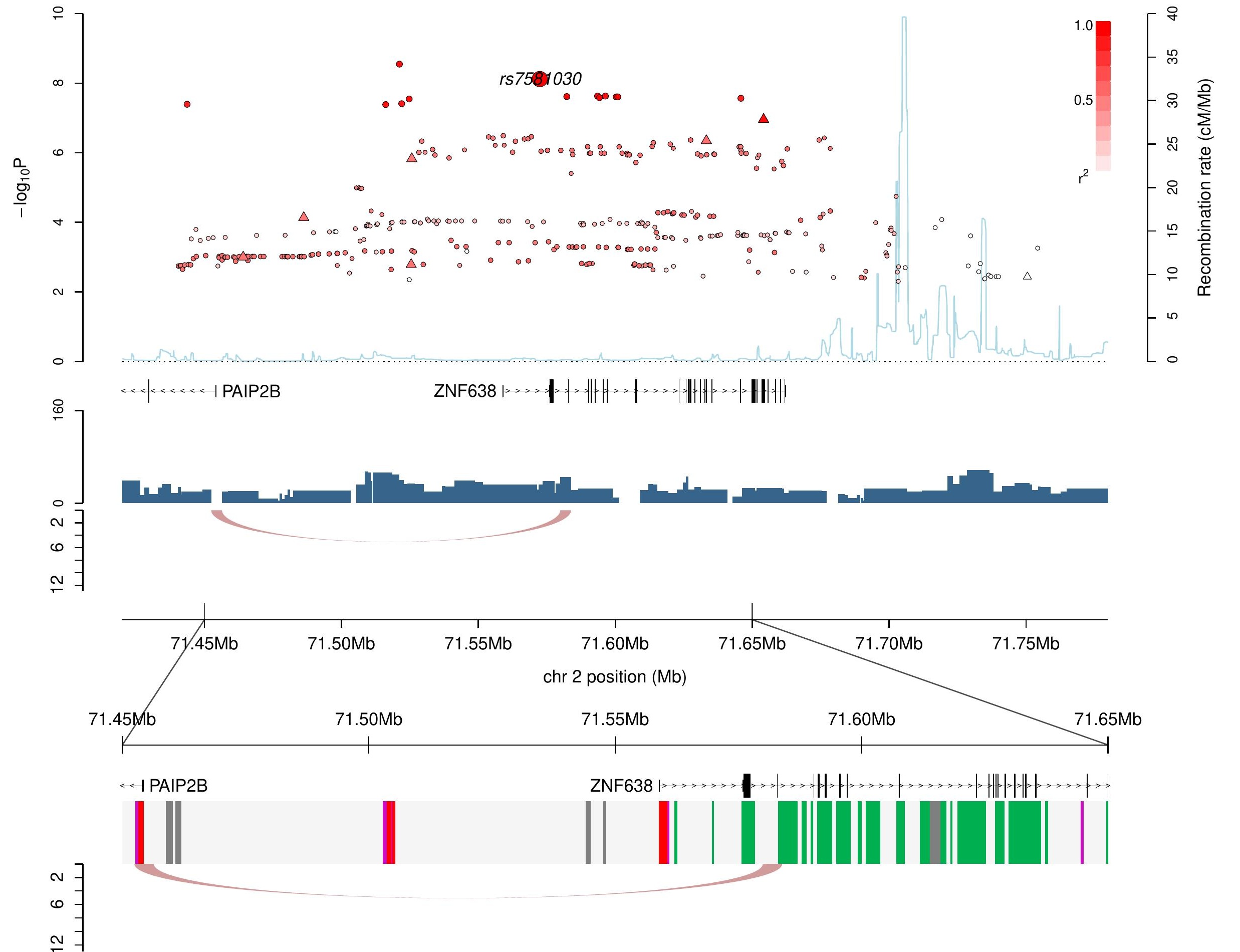




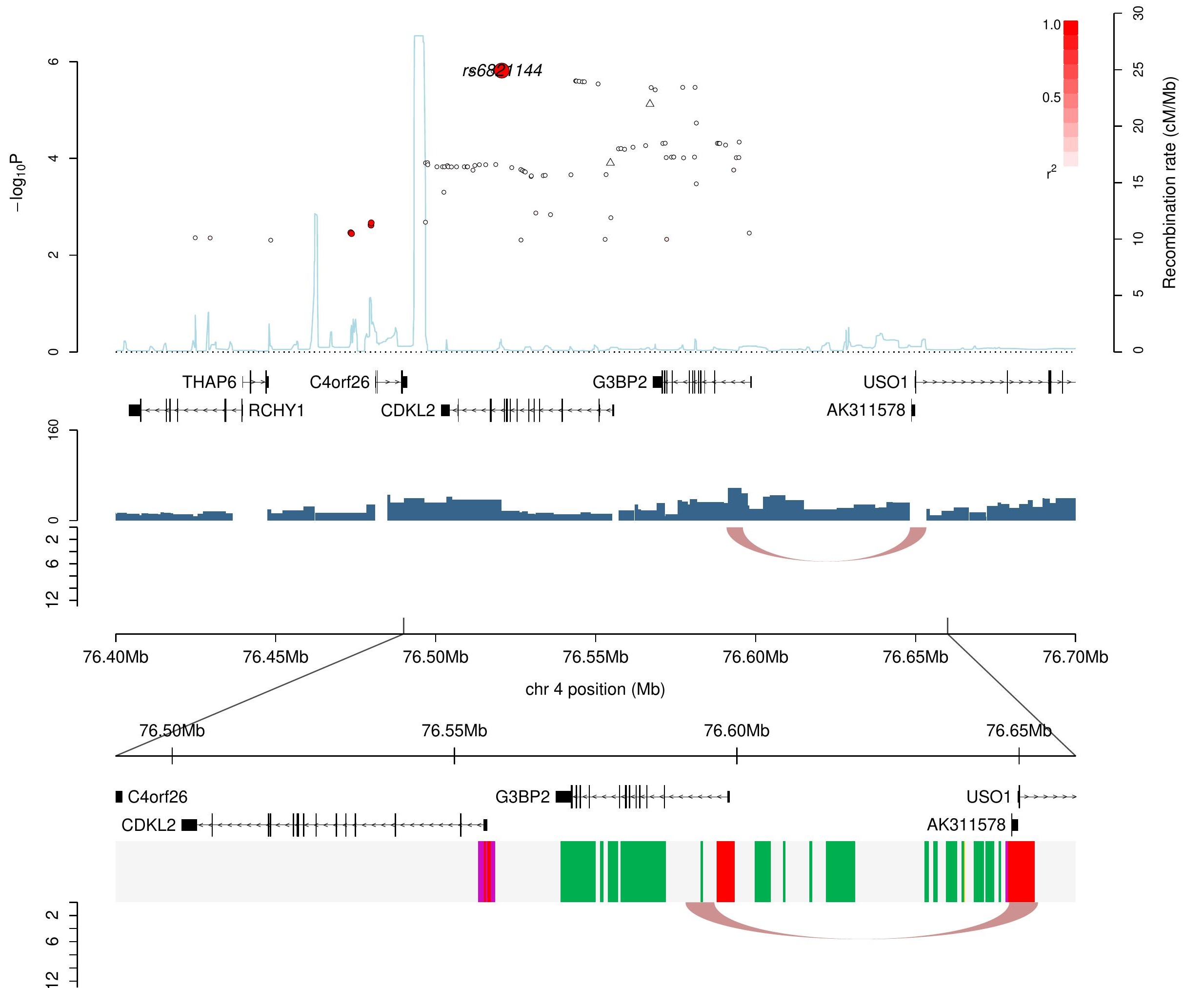



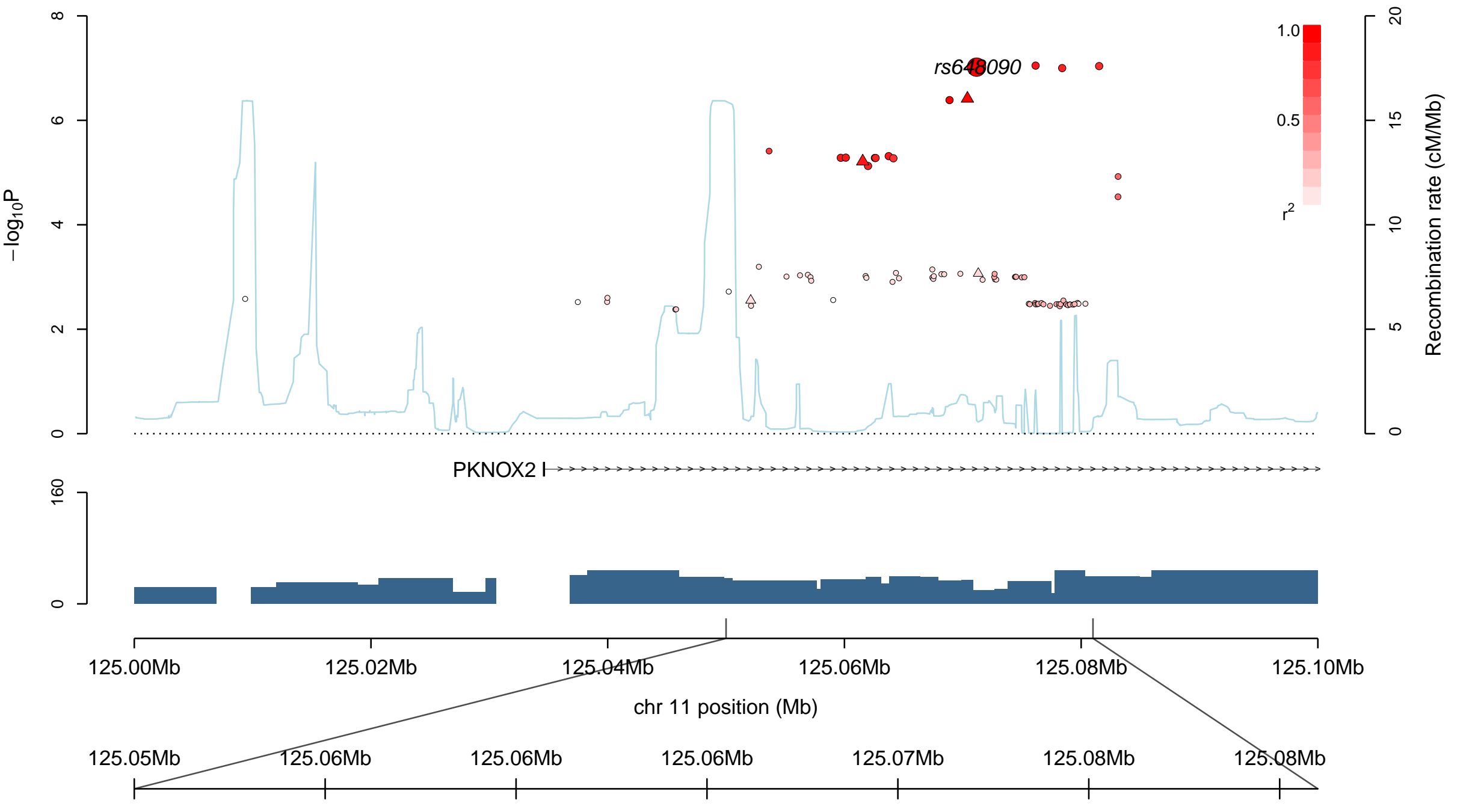


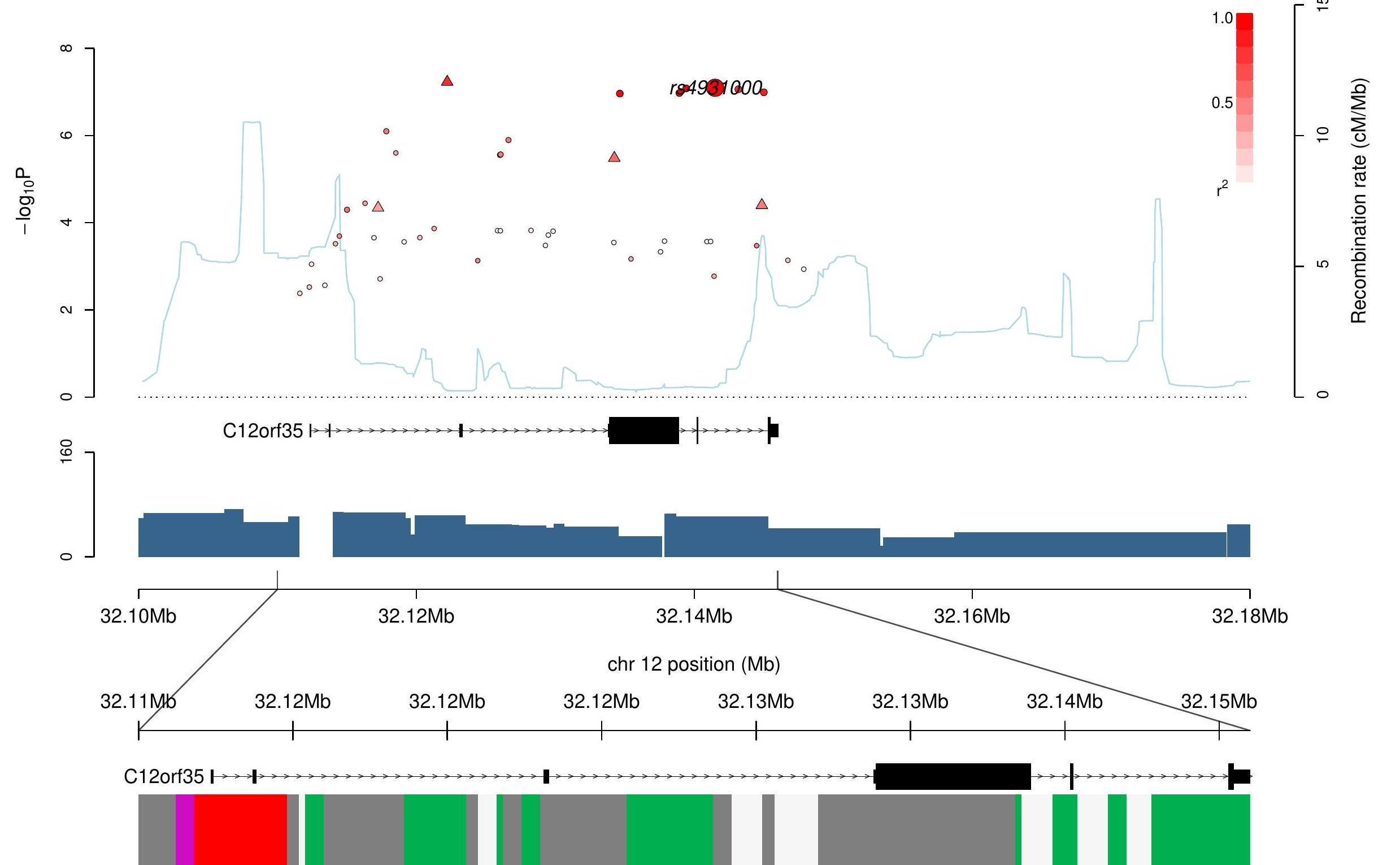




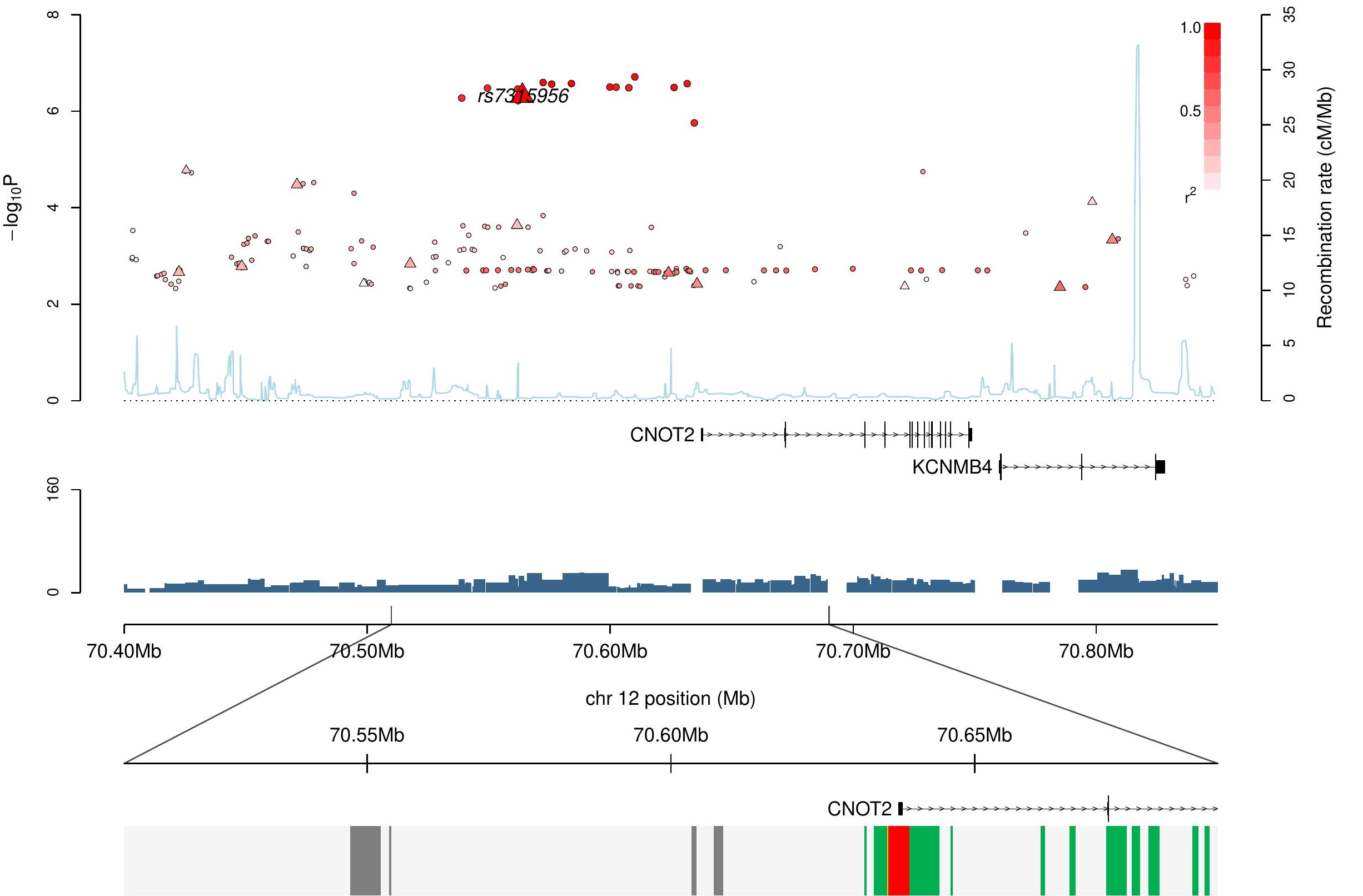




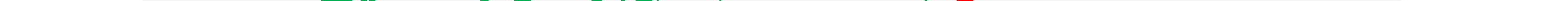




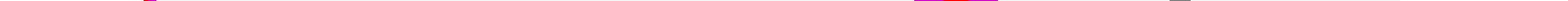




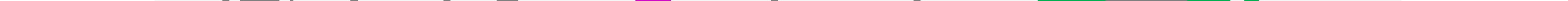




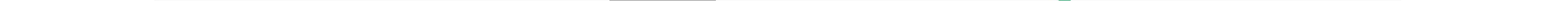




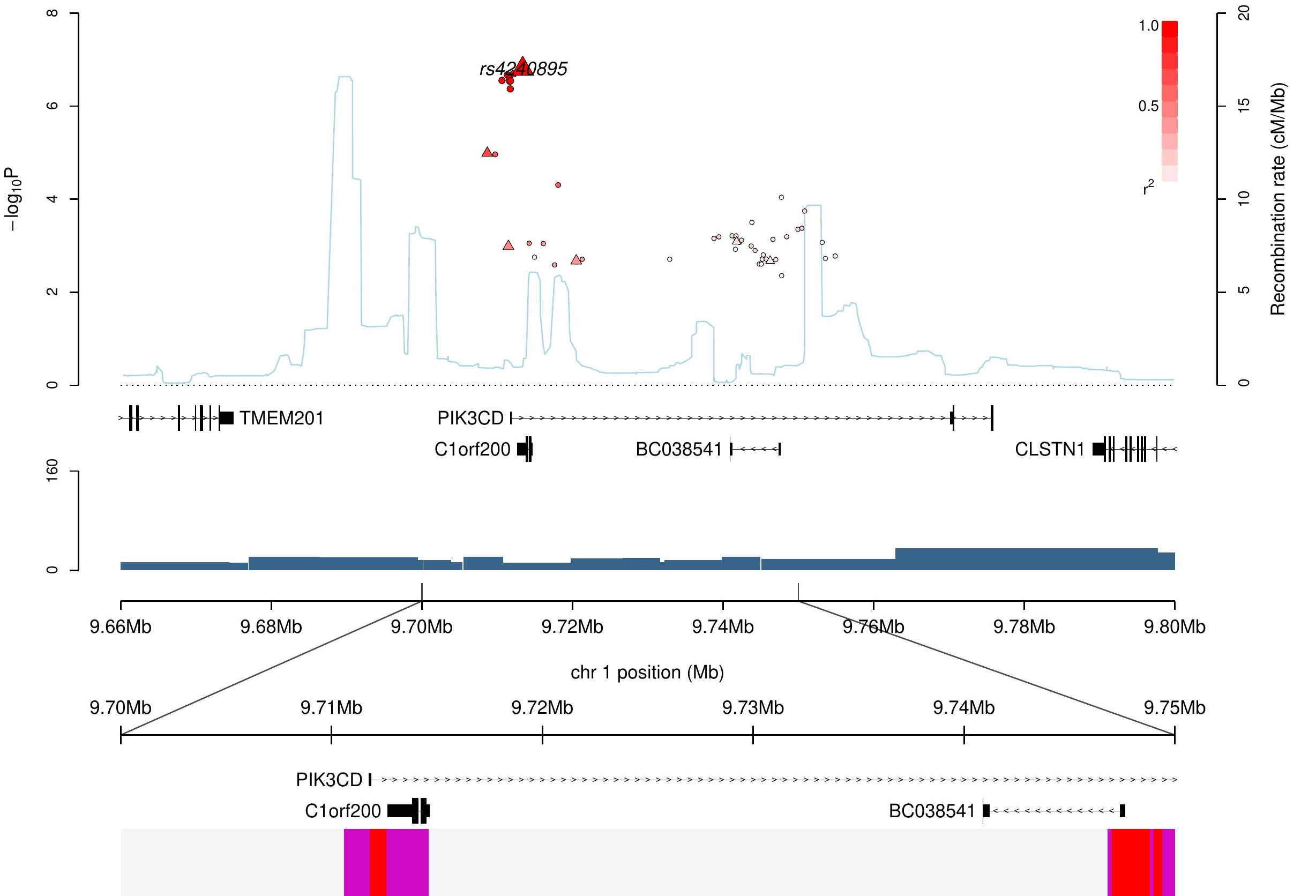




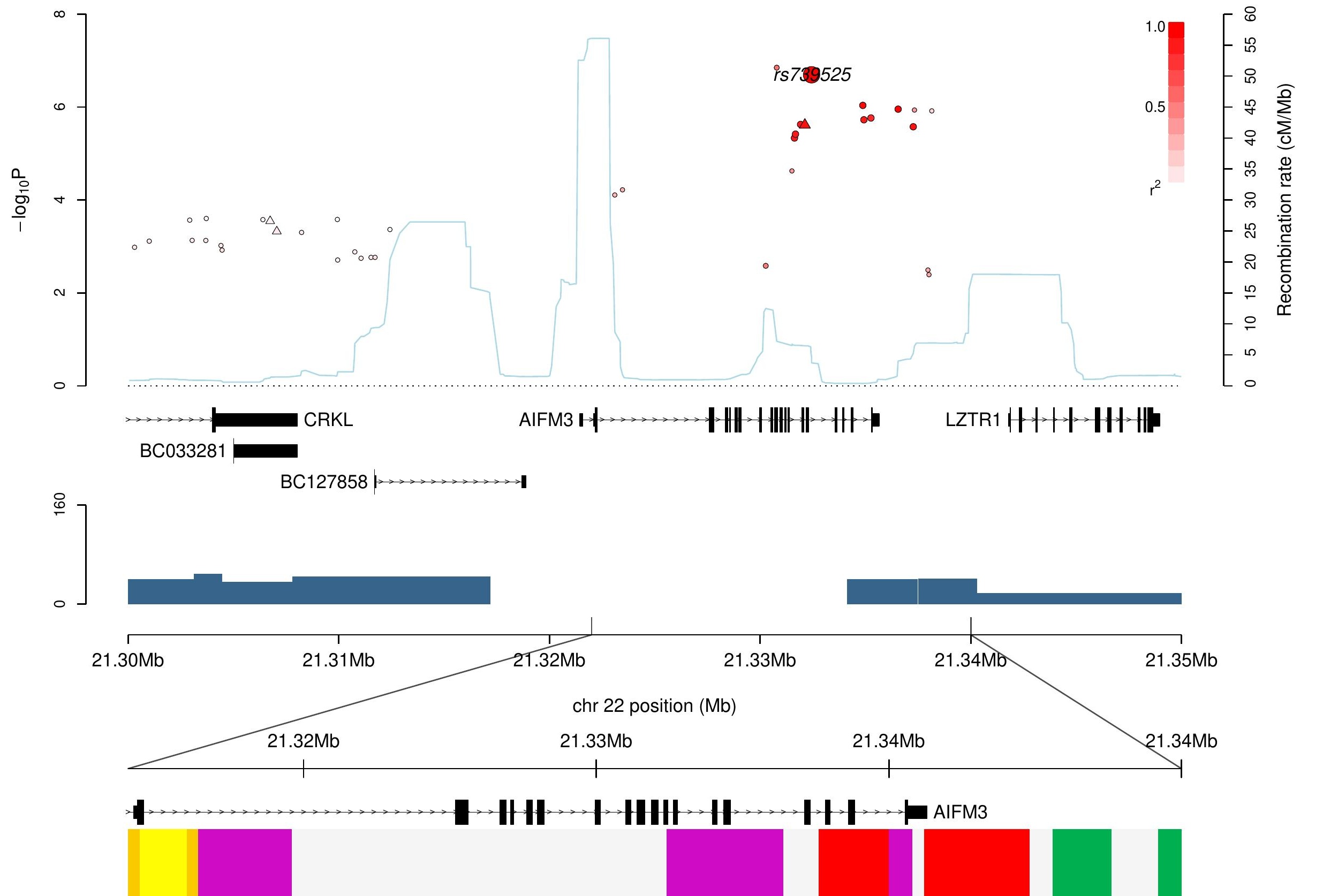




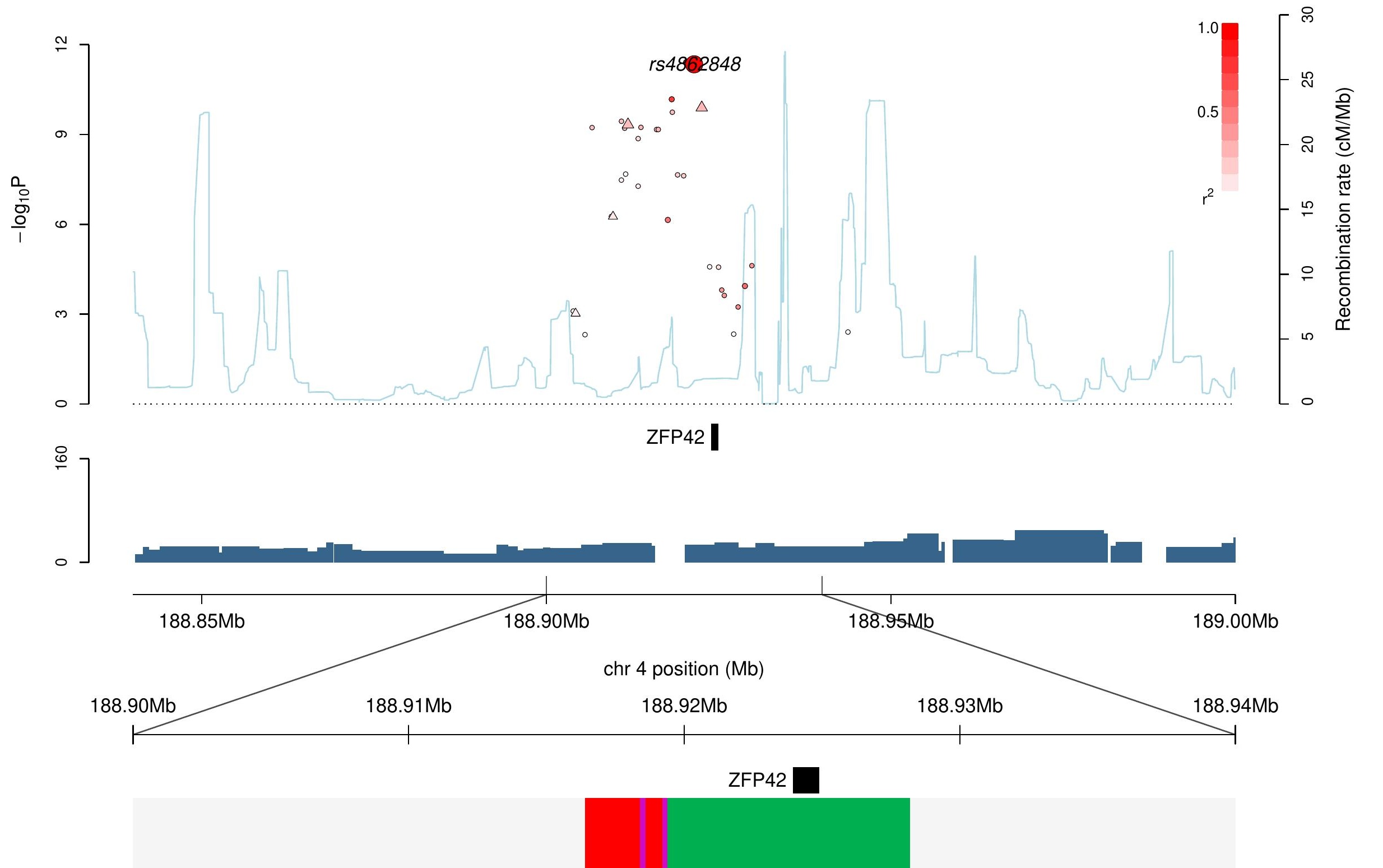




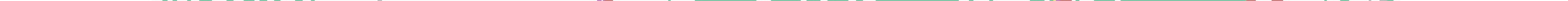




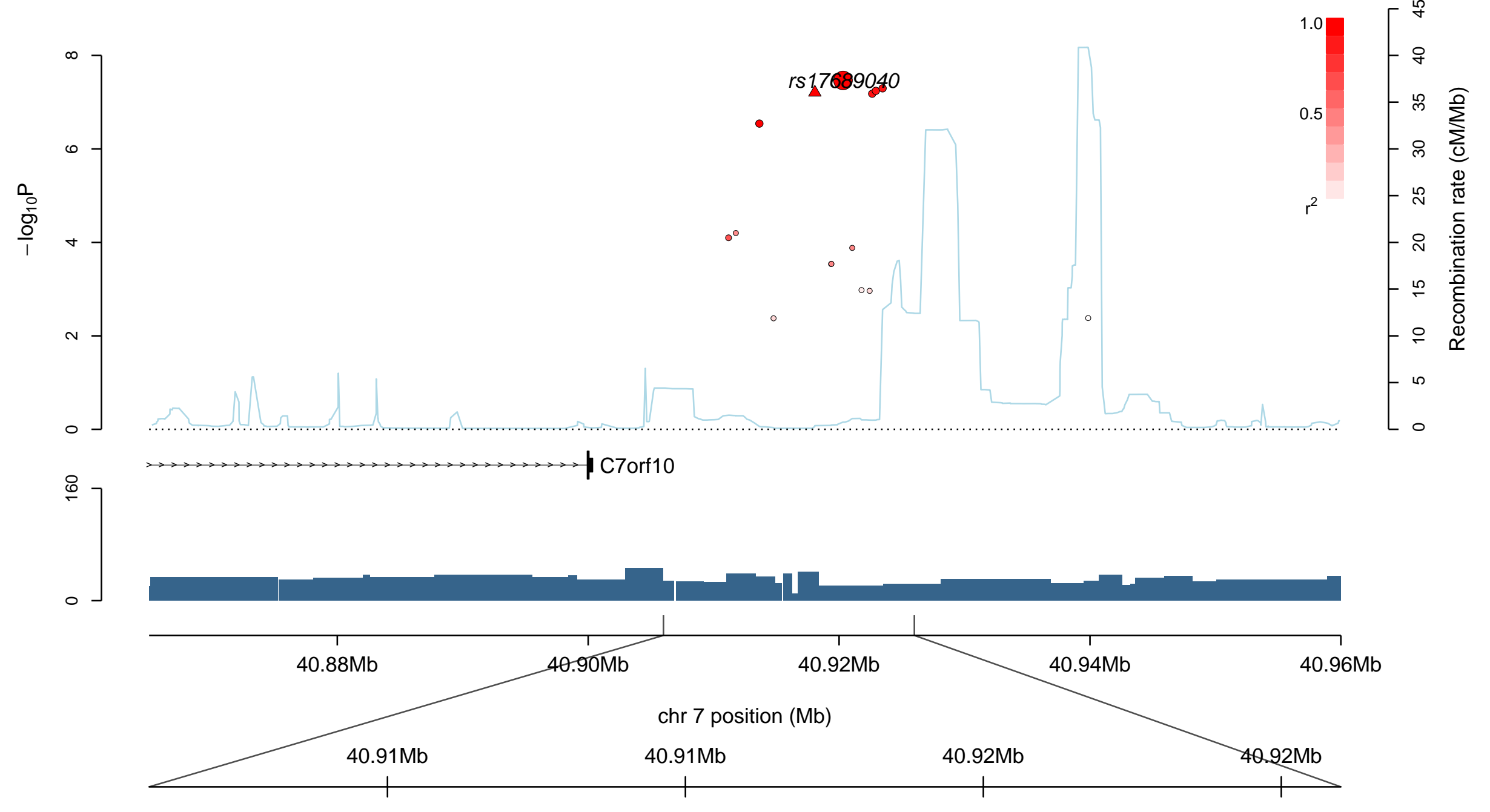


Supplementary Figure7 - Scatterplot of Hi-C interaction scores (- $\log$ (weighted $P$-value)) for independent biological replicates one and two.

NTERA2 capture Hi-C interaction scores by biological replicate

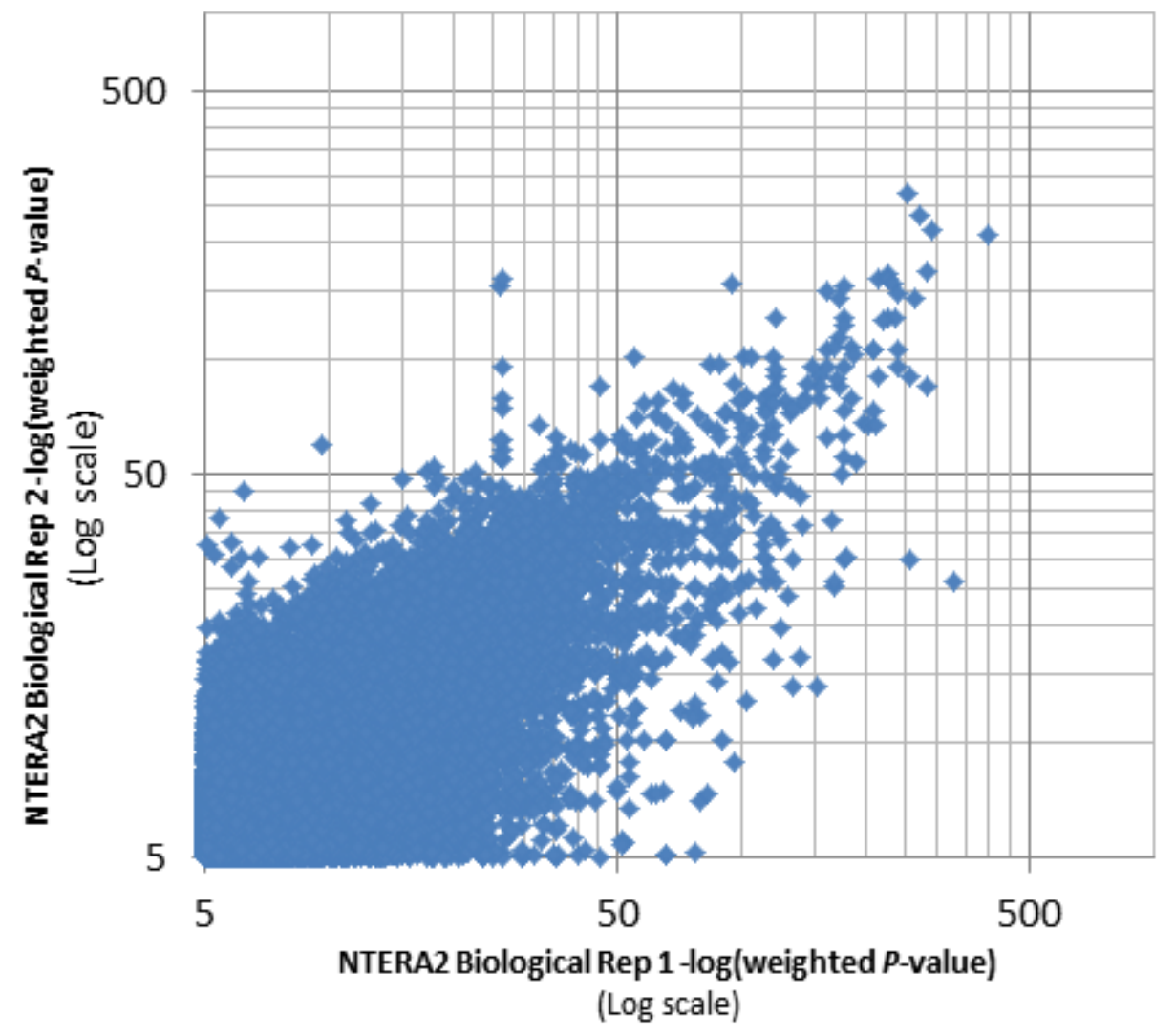


Supplementary Figure 8 - Density plot of $\mathrm{Hi}-\mathrm{C}$ interaction distances detected in this study compared to previously published data.

Density plot showing distribution of $\mathrm{CHi}-\mathrm{C}$ interaction distances

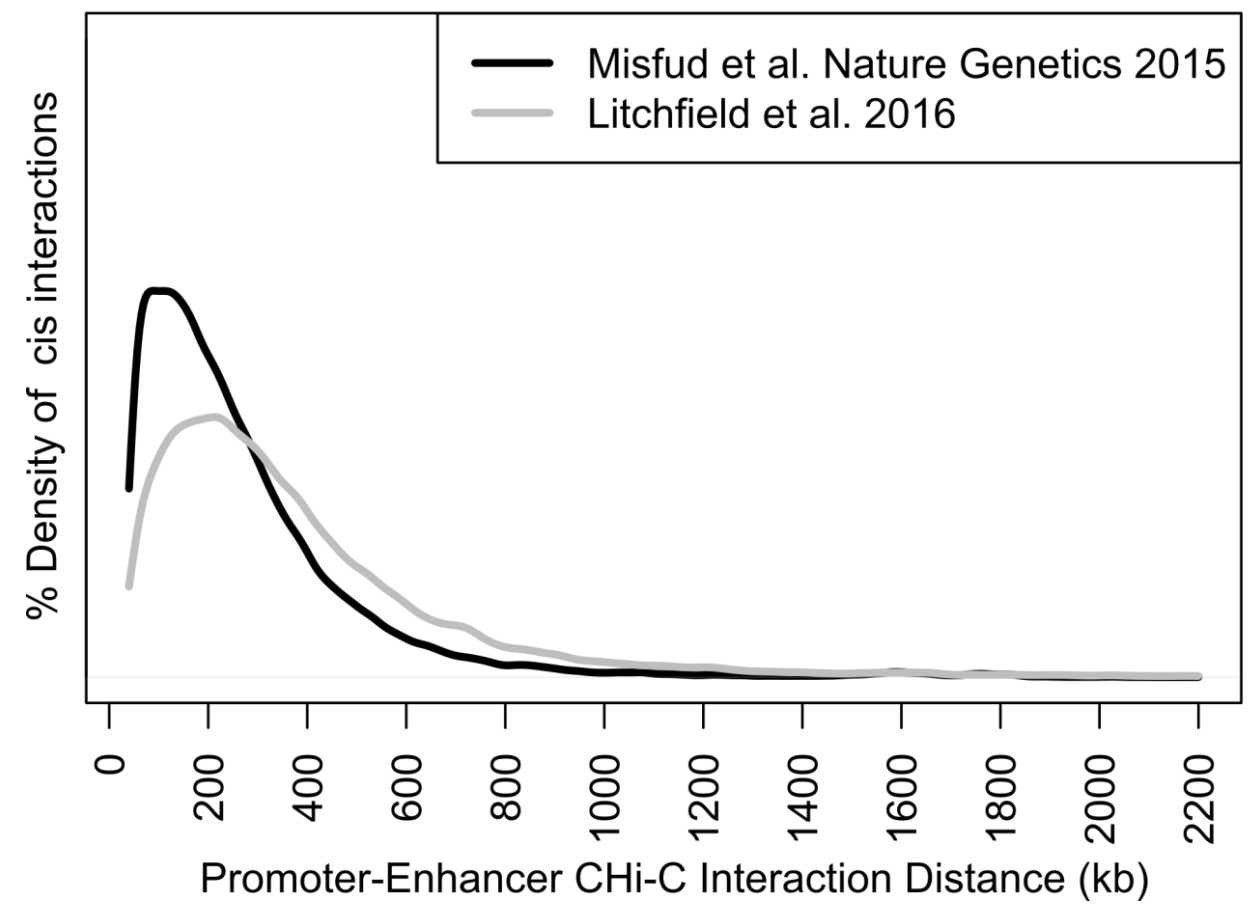


Supplementary Figure 9.

Validation of Hi-C data by 3C PCR assay.

Bar charts show the gel quantified relative interaction frequency between a given gene promoter and promoterinteracting Hindlll block (promoter-element, P-E) vs a control HindlII block (promoter-control, PC). Error bars represent the standard deviation of three replicates. Abbreviations: P-E, promoterelement; P-C, promotercontrol; L, ladder; B, BAC library; N1-3, NTERA2 3C libraries; NTC, no template control.

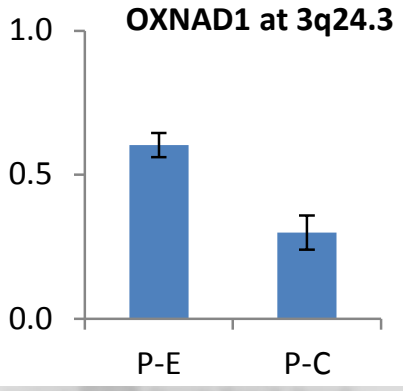

\section{P-E} $\mathrm{P}-\mathrm{C}$
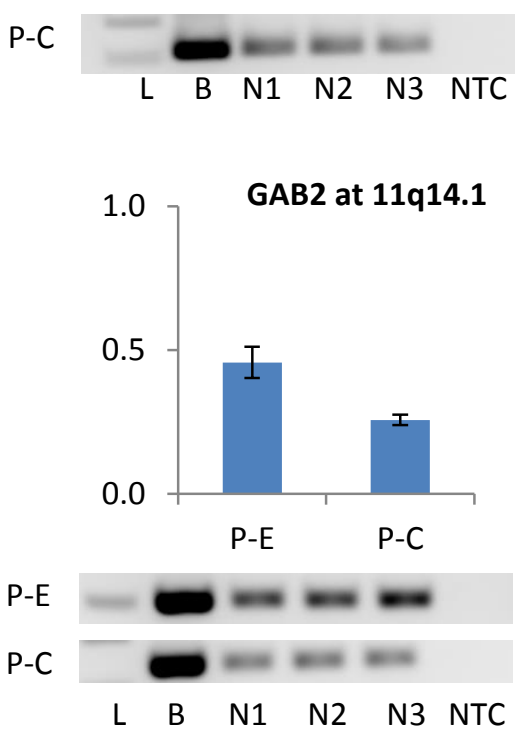

$\begin{array}{llllll}\text { L } & \text { B } & \text { N1 } & \text { N2 } & \text { N3 } & \text { NTC }\end{array}$
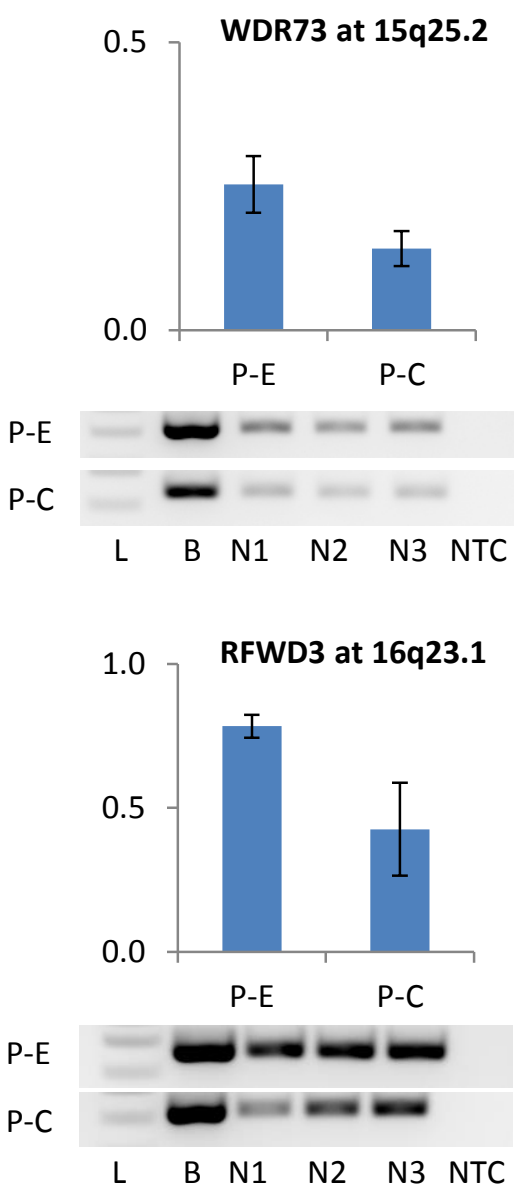

P-E

$\mathrm{P}-\mathrm{C}$
P-E

P-C
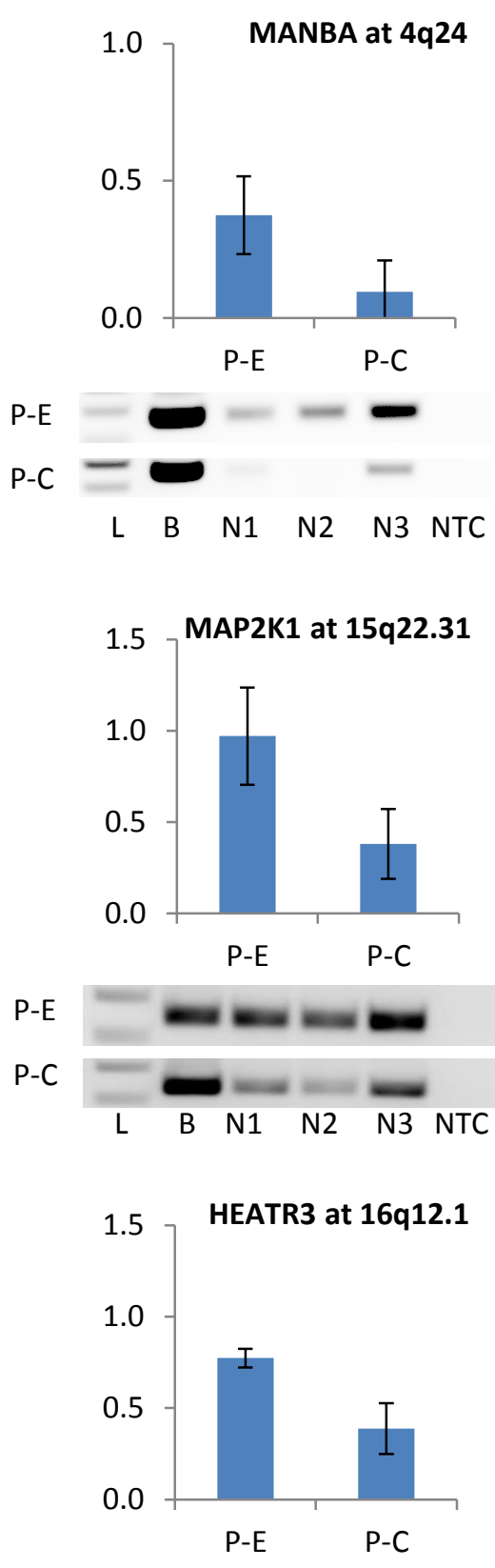

P-E

P-C

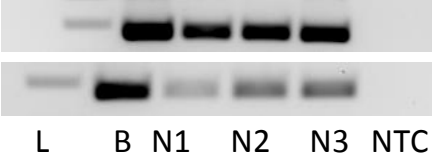


Supplementary Figure 10. Sanger chromatograms of P-E fragments of Chi-C interactions validated by $3 \mathrm{C}$ sequenced in an NTERA2 library. Promoters are shown to be ligated to their expected elements, separated by a Hindlll cutting site (between dotted lines).
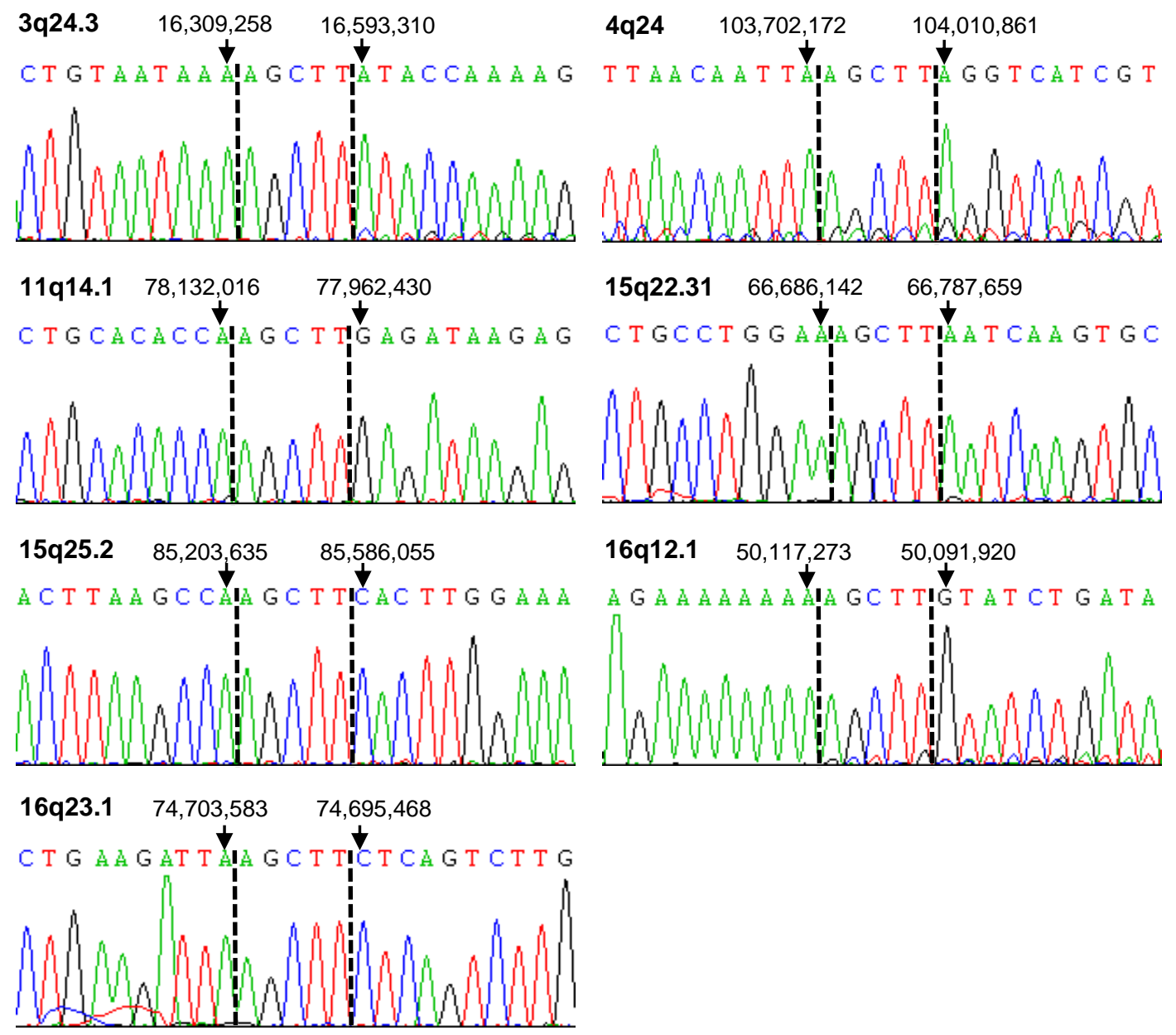
Supplementary Figure 11

Emission Parameters

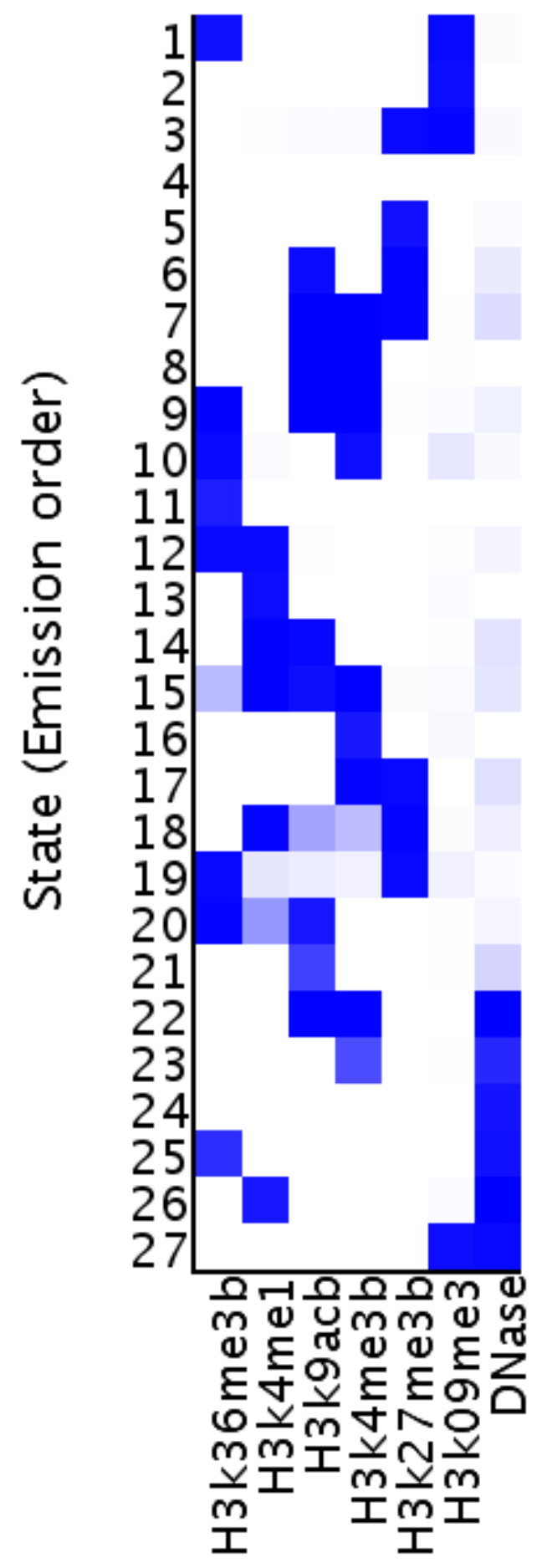

Mark 


\section{Supplementary note 1}

The UK Testicular Cancer Collaboration (UKTCC)

\begin{tabular}{|c|c|c|}
\hline Principal Investigator & Study Centre & Study centre address \\
\hline Rustin, Prof Gordon & Mount Vernon Hospital & $\begin{array}{l}\text { Mount Vernon Cancer Centre, Rickmansworth Road, Northwood, Middlesex, } \\
\text { HA6 2RN }\end{array}$ \\
\hline Srihari, Dr & Royal Shrewsbury Hospital & Trials Unit, Oncology Department, Mytton Oak Road, Shrewsbury, SY3 8XB \\
\hline Cole, Dr David & Great Western Hospital & 3rd Floor, Osprey Unit, Swindon, Wilts, SN3 6BB \\
\hline $\begin{array}{l}\text { Askill, Dr Colin \& Bertelli, Dr } \\
\text { Gianfilippo }\end{array}$ & $\begin{array}{l}\text { Singleton Hospital and Morriston } \\
\text { Hospital }\end{array}$ & SWW Cancer Institute, Sketty, Swansea, SA2 8QA \\
\hline Barber, Dr James & Velindre Hospital & $\begin{array}{l}\text { Clinical Trials Unit, Velindre Cancer Centre, Velindre Road, Whitchurch, } \\
\text { Cardiff CF14 2TL }\end{array}$ \\
\hline Gilby, Dr Ed & Royal United Hospital & Dept of Oncology and Haematology, Combe Park, Bath, BA1 3NG \\
\hline Huddart, Dr Robert & Royal Marsden Hospital Sutton & Downs Rd, Sutton, SM2 5PT \\
\hline White, Dr Jeff & Beatson Oncology Centre & $\begin{array}{l}\text { Beatson West of Scotland Cancer Centre, } 1053 \text { Great Western Road, Glasgow, } \\
\text { G11 0YN }\end{array}$ \\
\hline Braybrooke, Dr Jeremy & Bristol Haematology \& Oncology Centre & United Bristol Healthcare NHS trust, Horfield Rd, Bristol, BS2 8ED \\
\hline Leahy, Dr M and Welch, Dr R & Christie Hospital & Wilmslow Road, Withington, Manchester, M20 4BX \\
\hline
\end{tabular}




\begin{tabular}{|c|c|c|}
\hline Chakraborti, Dr P & Derbyshire Royal Infirmary & Derby Hospitals NHS Trust, London Road, Derby, DE1 2QY \\
\hline Joffe, Dr J & St James Hospital Leeds & Dept of Medical Oncology, Leeds, LS9 7TF \\
\hline Brown, Dr Richard & Wexham Park Hospital & Cancer Clinical Trials, John Ulster Post Grad Centre, Slough, Berks, SL2 4HL \\
\hline Faust, Dr Guy & Leicester Royal Infirmary & LNR Cancer Reseach Network, Knighton St, Leicester LE1 5WW \\
\hline Simmonds, Dr Peter & Southampton General Hospital & $\begin{array}{l}\text { Cancer Care Directorate, Medical Oncology, Mailpoint 306, Southampton } \\
\text { General Hospital, Tremona Rd, SO16 6YD }\end{array}$ \\
\hline Mazhar, Dr danish & Addenbrookes Hospital & $\begin{array}{l}\text { Addenbrookes Hospital, Cambridge Clinical Trials Centre, Oncology Clinical } \\
\text { Trials, (S4) Box 279, Hills Rd, CB2 0QQ }\end{array}$ \\
\hline $\begin{array}{l}\text { Stockdale, Dr A \& Hrouda, Dr D } \\
\text { \& Humber, Dr C. }\end{array}$ & University Hospital Walsgrave & $\begin{array}{l}\text { Arden Cancer Centre, West Wing, UHCW NHS trust, Clifford Bridge Rd, } \\
\text { Coventry, CV2 2DX }\end{array}$ \\
\hline Appel, Dr Wiebke & Royal Preston Hospital & $\begin{array}{l}\text { Dept of Oncology, Royal Preston Hospital, Sharoe Green Lane North, Fulwood } \\
\text { Preston, PR2 9HT }\end{array}$ \\
\hline Hong, Dr Anne & Royal Devon \& Exeter & $\begin{array}{l}\text { Exeter Oncology Centre, Royal Devon and Exeter Hospital, Barrack Rd, Exeter } \\
\text { EX2 5DW }\end{array}$ \\
\hline Dr Howard & Western General Hospital & $\begin{array}{l}\text { Scottish Cancer Research Network, Oncology Admin Corridor, Edinburgh } \\
\text { Cancer Centre, Western General Hospital, Crewe Rd South, Edinburgh, EH4 } \\
\text { 2XU }\end{array}$ \\
\hline Dr Fiona Douglas & Freeman Hospital & $\begin{array}{l}\text { Clinical Trials Unit, Newcastle General Hospital, Westgate Rd, Newcastle- } \\
\text { upon Tyne, NE4 6BE }\end{array}$ \\
\hline Bllomfield, Dr David & Royal Sussex County Hospital & Brighton and Sussex University Hospitals, The Sussex Cancer Centre, The \\
\hline
\end{tabular}




\begin{tabular}{|c|c|c|}
\hline & & Royal Sussex County Hospital, Eastern Road, Brighton, BN2 5BE \\
\hline Dr Mohammad Butt & Castle Hill Hospital & Castle Hill Hospital, Castle Road, Cottingham HU16 5JQ \\
\hline Dr Kay Kelly & Raigmore Hospital & Raigmore Hospital, Old Perth Road, Inverness, IV2 3UJ \\
\hline Dr R Mehra & New Cross Hospital & $\begin{array}{l}\text { Greater Midlands Cancer Research Network, The Chestnuts, The Royal } \\
\text { Wolverhampton Hospitals, New Cross Hospital NHS Trust, Wednesfield Road, } \\
\text { Wolverhampton, WV10 0QP }\end{array}$ \\
\hline Dr Richard Brown/Dr Paul Rogers & Royal Berkshire Hospital & $\begin{array}{l}\text { Royal Berkshire Hospital, Berkshire Cancer Centre, London Road, Reading, } \\
\text { Berkshire, RG1 5AN }\end{array}$ \\
\hline Chakraborti, Dr P & Queen's Hospital Burton & Queens Hospital, Burton upon Trent, Belvedere Road, Burton, DE13 0RB \\
\hline Dr Matthew Hatton & Weston Park Hospital & $\begin{array}{l}\text { Consultant Clinical Radiologist. Sheffield Teaching Hospitals NHS Foundation } \\
\text { Trust, } 8 \text { Beech Hill Road, Sheffield S10 2SB }\end{array}$ \\
\hline Hennig, Dr Ivo & Nottingham City Hospital & $\begin{array}{l}\text { Nottingham University Hospitals NHS Trust, City Hospital campus, Hucknall } \\
\text { Road, Nottingham, NG5 1PB }\end{array}$ \\
\hline Dr J McAteer & Belfast City Hospital & $\begin{array}{l}\text { Northern Ireland Cancer Centre, Belfast City Hospital, Lisburn Rd, Belfast, } \\
\text { BT9 7AB }\end{array}$ \\
\hline Dr Savage/Dr Seckl & Charing Cross Hospital & $\begin{array}{l}\text { Dept of Medical Oncology,Charing Cross, Fulham, Palace Rd, London W6 } \\
\text { 8RF }\end{array}$ \\
\hline Dr Joanna Gale & $\begin{array}{l}\text { Portsmouth Haematology \& Oncology } \\
\text { Centre }\end{array}$ & Level B Queen Alexandra Hospital, Cosham, Portsmouth, PO6 3LY \\
\hline
\end{tabular}




\begin{tabular}{|c|c|c|}
\hline Rustin, Prof Gordon & Hillingdon Hospital & $\begin{array}{l}\text { R\&D Office - Education Centre, Hillingdon Hospital, Pield Heath Road, } \\
\text { Hillingdon, UB8 3NN }\end{array}$ \\
\hline Prof Peter Clark & Royal Liverpool \& Broadgreen Hospitals & Prescot Street Liverpool, L78XP \\
\hline Dr Steve Woby & $\begin{array}{l}\text { Royal Oldham Hospital/Pennine Acute } \\
\text { Hospital }\end{array}$ & Roachdale Road Oldham OL1 2JH \\
\hline Dr Adrian Rathmell & James Cook Hospital & Middlesbrough TS4 3BW \\
\hline Dr Alan Lamont & Colchester/Essex County Hospital & Essex County \\
\hline Dr Guy Faust & Northampton General & Cliftonville, Northampton NN1 5BD \\
\hline Dr Naveed Sarwar & Basildon Hospital & Nethermayne Basildon Essex SS16 5NL \\
\hline Prof Nick Stuart & $\begin{array}{l}\text { Glan Clwyd Hospital and Ysbyty } \\
\text { Gwynedd }\end{array}$ & NW Cancer Treatment Centre, Glan Clwyd Hospital, LL18 5UJ \\
\hline Dr Simon Chowdhury & Guys \& St Thomas's & St Thomas Street, London SE1 9RT \\
\hline Dr Sharon Beesley & Maidstone and Tunbridge NHS Trust & Maidstone Hospital, Hemitage Lane, Barming, Maidstone, Kent ME16 9QQ \\
\hline Dr Winkler & West Middlesex University Hospital & $\begin{array}{l}\text { West Middlesex University Hospital NHS Trust, R\&D Department, 4th Floor, } \\
\text { East Wing,Twickenham Road, Isleworth Middlesex TW7 6AF }\end{array}$ \\
\hline Dr Abdel Hamid & Broomfield Hospital & $\begin{array}{l}\text { Broomfield Hospital, West Wing 2, Court Road, Broomfield, Chelmsford, } \\
\text { Essex CM1 7ET }\end{array}$ \\
\hline Dr Sanjeev Pathak & Doncaster Royal Infirmary & $\begin{array}{l}\text { Joint Research Office of Doncaster and Bassetlaw Hospitals NHS Foundation } \\
\text { Trust, First Floor 'C' Block, Doncaster Royal Infirmary, Armthorpe Road, } \\
\text { Doncaster DN2 5LT }\end{array}$ \\
\hline
\end{tabular}




\begin{tabular}{|l|l|l|}
\hline Dr Krishnaswamy Madhavan & Southend University Hospital NHS & Pittlewell Chase, Westcliff-On-Sea, Essex SSO 0RH \\
\hline Dr Martin Highley & Deundation Trust & Plymouth Hospitals NHS Trust, Derriford Hospital, Plymouth, PL6 8DH \\
\hline Dr Julian Money-Kyrle & Royal Surrey County Hospital & Royal Surrey County Hospital, St Lukes Cancer Centre, Egerton Road, \\
& Guildford, Surrey GU2 7XX \\
\hline Dr Cathryn Brock & Foundation Trust & Chelsea \& Westminster Hospital, Unit 101, 1st Floor, Harbour Yard, Chelsea \\
\hline Dr Thiagarajan Sreenivasan & United Linconshire Hospitals NHS Trust & Lincoln County Hospital, Greetwell Road, Lincoln, LN2 5QY \\
\hline Dr Thiagarajan Sreenivasan & United Linconshire Hospitals NHS Trust & Pilgrim Hospital, Boston, Lincolnshire PE21 9QS \\
\hline
\end{tabular}




\section{Supplementary note 2}

\section{The PRACTICAL Consortium (http://practical.ccge.medschl.cam.ac.uk/): OncoArray:}

Brian E. Henderson ${ }^{1}$, Christopher A. Haiman ${ }^{1}$, Sara Benlloch ${ }^{2,3}$, Fredrick R. Schumacher ${ }^{4,5}$, Ali Amin Al Olama ${ }^{2,6}$, Sonja I. Berndt ${ }^{7}$, David V. Conti ${ }^{1}$, Fredrik Wiklund ${ }^{8}$, Stephen Chanock ${ }^{7}$, Victoria L. Stevens ${ }^{9}$, Catherine M. Tangen ${ }^{10}$, Jyotsna Batra ${ }^{11,12}$, APCB BioResource ${ }^{11}$, Judith Clements $^{11,12}$, Henrik Gronberg ${ }^{8}$, Johanna Schleutker ${ }^{13,14,15}$, Demetrius Albanes ${ }^{7}$, Stephanie Weinstein ${ }^{7}$, Alicja Wolk ${ }^{16}$, Catharine West ${ }^{17}$, Lorelei Mucci ${ }^{18}$, Géraldine Cancel-Tassin ${ }^{19,20}$, Stella Koutros ${ }^{7}$, Karina Dalsgaard Sorensen ${ }^{21,22}$, Lovise Maehle ${ }^{23}$, David E. Neal ${ }^{24,25}$, Ruth C. Travis $^{26}$, Robert J. Hamilton ${ }^{27}$, Sue Ann Ingles ${ }^{1}$, Barry Rosenstein ${ }^{28,29}$, Yong-Jie Lu ${ }^{30}$, Graham G. Giles ${ }^{31,32}$, Adam S. Kibel ${ }^{33}$, Ana Vega ${ }^{34}$, Manolis Kogevinas ${ }^{35,36,37,38}$, Kathryn L. Penney ${ }^{39}$, Jong Y. Park ${ }^{40}$, Janet L. Stanford ${ }^{41,42}$, Cezary Cybulski ${ }^{43}$, Børge G.

Nordestgaard $^{44,45}$, Hermann Brenner ${ }^{46,47,48}$, Christiane Maier ${ }^{49}$, Jeri Kim ${ }^{50}$, Esther M. John ${ }^{51,52}$, Manuel R. Teixeira ${ }^{53,54}$, Susan L. Neuhausen ${ }^{55}$, Kim De Ruyck ${ }^{56}$, Azad Razack ${ }^{57}$, Lisa F. Newcomb ${ }^{41,58}$, Davor Lessel ${ }^{59}$, Radka Kaneva ${ }^{60}$, Nawaid Usmani ${ }^{61,62}$, Frank Claessens $^{63}$, Paul A. Townsend ${ }^{64}$, Manuela Gago Dominguez ${ }^{65,66}$, Monique J. Roobol ${ }^{67}$, Florence Menegaux ${ }^{68}$

1 Department of Preventive Medicine, Keck School of Medicine, University of Southern California/Norris Comprehensive Cancer Center, Los Angeles, CA, USA.

2 Centre for Cancer Genetic Epidemiology, Department of Public Health and Primary Care, University of Cambridge, Strangeways Research Laboratory, Cambridge, UK.

3 The Institute of Cancer Research, London, UK.

4 Department of Epidemiology and Biostatistics, Case Western Reserve University, Cleveland, OH, USA.

5 Seidman Cancer Center, University Hospitals, Cleveland, OH, USA.

6 University of Cambridge, Department of Clinical Neurosciences, Cambridge, UK.

7 Division of Cancer Epidemiology and Genetics, National Cancer Institute, NIH, Bethesda, MD, USA.

8 Department of Medical Epidemiology and Biostatistics, Karolinska Institute, Stockholm, Sweden.

9 Epidemiology Research Program, American Cancer Society, 250 Williams Street, Atlanta, GA, USA.

10 SWOG Statistical Center, Fred Hutchinson Cancer Research Center, Seattle, WA, USA.

11 Australian Prostate Cancer Research Centre-Qld, Institute of Health and Biomedical Innovation and School of Biomedical Science, Queensland University of Technology, Brisbane, Queensland, Australia.

12 Translational Research Institute, Brisbane, Queensland, Australia.

13 Department of Medical Biochemistry and Genetics, Institute of Biomedicine, University of Turku, Finland.

14 Tyks Microbiology and Genetics, Department of Medical Genetics, Turku University Hospital, Finland.

15 BioMediTech, University of Tampere, Tampere, Finland.

16 Division of Nutritional Epidemiology, Institute of Environmental Medicine, Karolinska Institutet, Sweden.

17 Institute of Cancer Sciences, University of Manchester, Manchester Academic Health Science Centre, Radiotherapy Related Research, The Christie Hospital NHS Foundation Trust, Manchester, UK.

18 Department of Epidemiology, Harvard School of Pubic Health, Boston, MA, USA.

19 CeRePP, Pitie-Salpetriere Hospital, Paris, France.

20 UPMC Univ Paris 06, GRC N5 ONCOTYPE-URO, CeRePP, Tenon Hospital, Paris, France. 
21 Department of Molecular Medicine, Aarhus University Hospital, Denmark.

22 Department of Clinical Medicine, Aarhus University, Denmark.

23 Department of Medical Genetics, Oslo University Hospital, Norway.

24 University of Cambridge, Department of Oncology, Addenbrooke's Hospital, Cambridge, UK.

25 Cancer Research UK Cambridge Research Institute, Li Ka Shing Centre, Cambridge, UK.

26 Cancer Epidemiology, Nuffield Department of Population Health University of Oxford, Oxford, UK.

27 Dept. of Surgical Oncology, Princess Margaret Cancer Centre, Toronto, Canada.

28 Department of Radiation Oncology, Icahn School of Medicine at Mount Sinai, New York, NY, USA.

29 Department of Genetics and Genomic Sciences, Icahn School of Medicine at Mount Sinai, New York, NY, USA.

30 Centre for Molecular Oncology, Barts Cancer Institute, Queen Mary University of London, John Vane Science Centre, London, UK.

31 Cancer Epidemiology Centre, The Cancer Council Victoria, Melbourne, Victoria, Australia.

32 Centre for Epidemiology and Biostatistics, Melbourne School of Population and Global Health, The University of Melbourne, Melbourne, Australia.

33 Division of Urologic Surgery, Brigham and Womens Hospital, Boston, MA, USA.

34 Fundación Pública Galega de Medicina Xenómica-SERGAS, Grupo de Medicina Xenómica, CIBERER, IDIS, Santiago de Compostela, Spain.

35 Centre for Research in Environmental Epidemiology (CREAL), Barcelona Institute for Global Health (ISGlobal), Barcelona, Spain.

36 CIBER Epidemiología y Salud Pública (CIBERESP), Madrid, Spain.

37 IMIM (Hospital del Mar Research Institute), Barcelona, Spain.

38 Universitat Pompeu Fabra (UPF), Barcelona, Spain.

39 Channing Division of Network Medicine, Department of Medicine, Brigham and Women's Hospital/Harvard Medical School, Boston, MA, USA.

40 Department of Cancer Epidemiology, Moffitt Cancer Center, Tampa, USA.

41 Division of Public Health Sciences, Fred Hutchinson Cancer Research Center, Seattle, Washington, USA.

42 Department of Epidemiology, School of Public Health, University of Washington, Seattle, Washington, USA.

43 International Hereditary Cancer Center, Department of Genetics and Pathology, Pomeranian Medical University, Szczecin, Poland.

44 Faculty of Health and Medical Sciences, University of Copenhagen, Denmark.

45 Department of Clinical Biochemistry, Herlev and Gentofte Hospital, Copenhagen University Hospital, Herlev, Denmark.

46 Division of Clinical Epidemiology and Aging Research, German Cancer Research Center (DKFZ), Heidelberg, Germany.

47 German Cancer Consortium (DKTK), German Cancer Research Center (DKFZ), Heidelberg, Germany.

48 Division of Preventive Oncology, German Cancer Research Center (DKFZ) and National Center for Tumor Diseases (NCT), Heidelberg, Germany.

49 Institute for Human Genetics, University Hospital UIm, Ulm, Germany.

50 The University of Texas M. D. Anderson Cancer Center, Department of Genitourinary Medical Oncology, Houston, TX, USA.

51 Cancer Prevention Institute of California, Fremont, CA, USA.

52 Department of Health Research \& Policy (Epidemiology) and Stanford Cancer Institute, Stanford University School of Medicine, Stanford, CA, USA.

53 Department of Genetics, Portuguese Oncology Institute of Porto, Porto, Portugal.

54 Biomedical Sciences Institute (ICBAS), University of Porto, Porto, Portugal.

55 Department of Population Sciences, Beckman Research Institute of the City of Hope, Duarte, CA, USA. 
56 Ghent University, Faculty of Medicine and Health Sciences, Basic Medical Sciences, Gent, Belgium.

57 Department of Surgery, Faculty of Medicine, University of Malaya, Kuala Lumpur, Malaysia.

58 Department of Urology, University of Washington, Seattle, WA, USA.

59 Institute of Human Genetics, University Medical Center Hamburg-Eppendorf, Hamburg, Germany.

60 Molecular Medicine Center, Department of Medical Chemistry and Biochemistry, Medical University, Sofia, Bulgaria.

61 Department of Oncology, Cross Cancer Institute, University of Alberta, Edmonton, Alberta, Canada.

62 Division of Radiation Oncology, Cross Cancer Institute, Edmonton, Alberta, Canada.

63 Molecular Endocrinology Laboratory, Department of Cellular and Molecular Medicine, KU Leuven, Leuven, Belgium.

64 Institute of Cancer Sciences, Manchester Cancer Research Centre, University of Manchester, Manchester Academic Health Science Centre, St Mary's Hospital, Manchester, UK.

65 Genomic Medicine Group, Galician Foundation of Genomic Medicine, Instituto de Investigacion Sanitaria de Santiago de Compostela (IDIS), Complejo Hospitalario Universitario de Santiago, Servicio Galego de Saúde, SERGAS, Santiago De Compostela, Spain.

66 University of California San Diego, Moores Cancer Center, La Jolla, CA, USA.

67 Department of Urology, Erasmus University Medical Center, Rotterdam, the Netherlands.

68 Cancer \& Environment Group, Center for Research in Epidemiology and Population Health (CESP), INSERM, University Paris-Sud, University Paris-Saclay, Villejuif, France.

69 Royal Marsden NHS Foundation Trust, London, UK. 